\title{
Indecomposable sets of finite perimeter in doubling metric measure spaces
}

\author{
Paolo Bonicatto ${ }^{1}$ Enrico Pasqualetto ${ }^{2}$ Tapio Rajala ${ }^{2}$
}

Received: 18 August 2019 / Accepted: 31 January 2020 / Published online: 4 March 2020

(c) The Author(s) 2020

\begin{abstract}
We study a measure-theoretic notion of connectedness for sets of finite perimeter in the setting of doubling metric measure spaces supporting a weak $(1,1)$-Poincaré inequality. The two main results we obtain are a decomposition theorem into indecomposable sets and a characterisation of extreme points in the space of BV functions. In both cases, the proof we propose requires an additional assumption on the space, which is called isotropicity and concerns the Hausdorff-type representation of the perimeter measure.
\end{abstract}

Mathematics Subject Classification 26B30 · 53C23

\section{Contents}

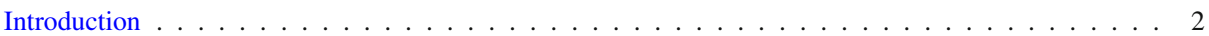

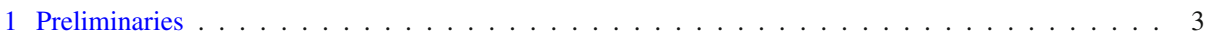

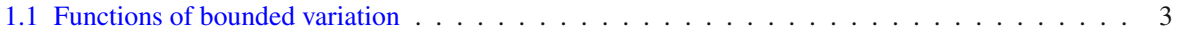

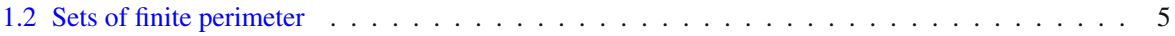

1.3 Fine properties of sets of finite perimeter in PI spaces . . . . . . . . . . . . . . . . 6

2 Decomposability of a set of finite perimeter . . . . . . . . . . . . . . . . . . . . . 14

2.1 Definition of decomposable set and its basic properties . . . . . . . . . . . . . . . . . 14

2.2 Decomposition theorem . . . . . . . . . . . . . . . . . . . . . . . 19

3 Extreme points in the space of BV functions . . . . . . . . . . . . . . . . 25

3.1 Simple sets and extreme points in BV . . . . . . . . . . . . . . . . . . . . 25

3.2 Holes and saturation . . . . . . . . . . . . . . . . . . . . . . . . 30

Communicated by A. Malchiodi.

Enrico Pasqualetto

enrico.e.pasqualetto@jyu.fi

Paolo Bonicatto

paolo.bonicatto@unibas.ch

Tapio Rajala

tapio.m.rajala@jyu.fi

1 Departement Mathematik und Informatik, Universität Basel, Spiegelgasse 1, 4051 Basel, Switzerland

2 Department of Mathematics and Statistics, University of Jyvaskyla, P.O. Box 35 (MaD), 40014 Jyvaskyla, Finland 
4 Alternative proof of the decomposition theorem f . . . . . . . . . . . . . . 32

Appendix A: Extreme points . . . . . . . . . . . . . . . . . . . . . . . 36

Appendix B: Lyapunov vector-measure theorem . . . . . . . . . . . . . . . . . . . . . . . . . . . . 36

References . . . . . . . . . . . . . . . . . . . . . . . 38

\section{Introduction}

The classical Euclidean theory of functions of bounded variation and sets of finite perimeter-whose cornerstones are represented, for instance, by $[6,15,17,22,29,36]$ - has been successfully generalised in different directions, to several classes of metric structures. Amongst the many important contributions in this regard, we just single out the pioneering works $[9-11,16,25,28]$. Although the basic theory of BV functions can be developed on abstract metric measure spaces (see, e.g., [5]), it is in the framework of doubling spaces supporting a weak $(1,1)$-Poincaré inequality (in the sense of Heinonen-Koskela [30]) that quite a few fine properties are satisfied (see $[1,2,38]$ ).

The aim of the present paper is to study the notion of indecomposable set of finite perimeter on doubling spaces supporting a weak $(1,1)$-Poincaré inequality (that we call PI spaces for brevity). By indecomposable set we mean a set of finite perimeter $E$ that cannot be written as disjoint union of two non-negligible sets $F, G$ satisfying $\mathrm{P}(E)=\mathrm{P}(F)+\mathrm{P}(G)$. This concept constitutes the measure-theoretic counterpart to the topological notion of 'connected set' and, as such, many statements concerning connectedness have a correspondence in the context of indecomposable sets.

In the Euclidean framework, the main properties of indecomposable sets have been systematically investigated by Ambrosio et al. in [4]. The results of this paper are mostly inspired by (and actually extend) the contents of [4]. In the remaining part of the Introduction, we will briefly describe our two main results: the decomposition theorem for sets of finite perimeter and the characterisation of extreme points in the space of BV functions. In both cases, the natural setting to work in is that of PI spaces satisfying an additional condition-called isotropicity — which we are going to describe in the following paragraph.

Let $(\mathrm{X}, d, \mathfrak{m})$ be a PI space and $E \subset \mathrm{X}$ a set of finite perimeter; we refer to Sect. 1 for the precise definition of perimeter and the terminology used in the following. The perimeter measure $\mathrm{P}(E, \cdot)$ associated to $E$ can be written as $\theta_{E} \mathcal{H}\left\llcorner\partial^{e} E\right.$, where $\mathcal{H}$ stands for the codimension-one Hausdorff measure on $\mathrm{X}$, while $\partial^{e} E$ is the essential boundary of $E$ (i.e., the set of points where neither the density of $E$ nor that of its complement vanishes) and $\theta_{E}: \partial^{e} E \rightarrow[0,+\infty)$ is a suitable density function; $\mathrm{cf}$. Theorem 1.23. The integral representation formula was initially proven in [1] only for Ahlfors-regular spaces, and this additional assumption has been subsequently removed in [2]. It is worth to point out that the weight function $\theta_{E}$ might (and, in some cases, does) depend on the set $E$ itself; see, for instance, Example 1.27. In this paper, we mainly focus our attention on those PI spaces where $\theta_{E}$ is independent of $E$, which are said to be isotropic (the terminology comes from [7]). As we will discuss in Example 1.31, the class of isotropic PI spaces includes weighted Euclidean spaces, Carnot groups of step 2 and non-collapsed $R C D$ spaces. Another key feature of the theory of sets of finite perimeter in PI spaces is given by the relative isoperimetric inequality (see Theorem 1.17 below), which has been obtained by M. Miranda in the paper [38].

Our main result (namely, Theorem 2.14) states that on isotropic PI spaces any set of finite perimeter $E$ can be written as (finite or countable) disjoint union of indecomposable sets. Moreover, these components-called essential connected components of E-are uniquely determined and maximal with respect to inclusion, meaning that any indecomposable subset 
of $E$ must be contained (up to null sets) in one of them. We propose two different proofs of this decomposition result, in Sects. 2 and 4, respectively. The former is a variational argument that was originally carried out in [4], while the latter is adapted from [33] and based on Lyapunov's convexity theorem. However, both approaches strongly rely upon three fundamental ingredients: representation formula for the perimeter measure, relative isoperimetric inequality, and isotropicity. We do not know whether the last one is in fact needed for the decomposition to hold (see also Example 2.16).

Furthermore, in Sect. 3 we study the extreme points in the space BV(X) of functions of bounded variation defined over $\mathrm{X}$; we are again assuming $(\mathrm{X}, d, \mathfrak{m})$ to be an isotropic PI space. More precisely: call $\mathcal{K}(\mathrm{X} ; K)$ the family of all those functions $f \in \mathrm{BV}(\mathrm{X})$ supported in $K$, whose total variation satisfies $|D f|(\mathrm{X}) \leq 1$ (where $K \subset \mathrm{X}$ is a fixed compact set). Then we can completely characterise (under a few additional assumptions) the extreme points of $\mathcal{K}(\mathrm{X} ; K)$ as a convex, compact subset of $L^{1}(\mathfrak{m})$; see Theorem 3.8. It turns out that these extreme points coincide (up to a sign) with the normalised characteristic functions of simple sets (cf. Definition 3.1). In the Euclidean case, the very same result was proven by W. H. Fleming in $[23,24]$ (see also [13]). Part of Sect. 3 is dedicated to some equivalent definitions of simple set: in the general framework of isotropic PI spaces, a plethora of phenomena concerning simple sets may occur, differently from what happens in $\mathbb{R}^{n}$ (see [4]). For more details, we refer to the discussion at the beginning of Sect. 3.1.

\section{Preliminaries}

For our purposes, by metric measure space we mean a triple $(\mathrm{X}, d, \mathfrak{m})$, where $(\mathrm{X}, d)$ is a complete and separable metric space, while $\mathfrak{m} \neq 0$ is a non-negative, locally finite Borel measure on X. For any open set $\Omega \subset \mathrm{X}$ we denote by $\operatorname{LIP}_{\text {loc }}(\Omega)$ the space of all $\mathbb{R}$-valued locally Lipschitz functions on $\Omega$, while $\operatorname{LIP}_{\mathrm{bs}}(\mathrm{X})$ is the family of all those Lipschitz functions $f: \mathrm{X} \rightarrow \mathbb{R}$ whose support $\operatorname{spt}(f)$ is bounded. Given any $f \in \operatorname{LIP}_{\text {loc }}(\mathrm{X})$, we define the functions $\operatorname{lip}(f), \operatorname{lip}_{a}(f): X \rightarrow[0,+\infty)$ as

$$
\operatorname{lip}(f)(x):=\varlimsup_{y \rightarrow x} \frac{|f(y)-f(x)|}{d(y, x)}, \quad \operatorname{lip}_{a}(f)(x):=\varlimsup_{y, z \rightarrow x} \frac{|f(y)-f(z)|}{d(y, z)}
$$

whenever $x \in \mathrm{X}$ is an accumulation point, and $\operatorname{lip}(f)(x), \operatorname{lip}_{a}(f)(x):=0$ elsewhere. We call lip $(f)$ and $\operatorname{lip}_{a}(f)$ the local Lipschitz constant and the asymptotic Lipschitz constant of $f$, respectively.

We denote by $L^{0}(\mathfrak{m})$ the family of all real-valued Borel functions on $\mathrm{X}$, considered up to $\mathfrak{m}$-a.e. equality. For any given exponent $p \in[1, \infty]$, we indicate by $L^{p}(\mathfrak{m}) \subset L^{0}(\mathfrak{m})$ and $L_{\text {loc }}^{p}(\mathfrak{m}) \subset L^{0}(\mathfrak{m})$ the spaces of all $p$-integrable functions and locally $p$-integrable functions, respectively. Given an open set $\Omega \subset \mathrm{X}$ and any $E \subset \Omega$, we write $E \Subset \Omega$ to specify that $E$ is bounded and $\operatorname{dist}(E, X \backslash \Omega)>0$.

\subsection{Functions of bounded variation}

In the framework of general metric measure spaces, the definition of function of bounded variation - which is typically abbreviated to 'BV function' - has been originally introduced in [38] and is based upon a relaxation procedure. Let us recall it: 
Definition 1.1 (Function of bounded variation) Let $(\mathrm{X}, d, \mathfrak{m})$ be a metric measure space. Fix any function $f \in L_{\text {loc }}^{1}(\mathfrak{m})$. Given any open set $\Omega \subset \mathrm{X}$, we define the total variation of $f$ on $\Omega$ as

$$
|D f|(\Omega):=\inf \left\{\underset{n \rightarrow \infty}{\lim _{\Omega}} \int_{\Omega} \operatorname{lip}\left(f_{n}\right) \mathrm{dm} \mid\left(f_{n}\right)_{n} \subset \operatorname{LIP}_{\mathrm{loc}}(\Omega), f_{n} \rightarrow f \text { in } L_{\mathrm{loc}}^{1}(\mathfrak{m}\llcorner\Omega)\} .\right.
$$

Then $f$ is said to be of bounded variation-briefly, $f \in \mathrm{BV}(\mathrm{X})$-if $f \in L^{1}(\mathfrak{m})$ and $|D f|(\mathrm{X})<+\infty$.

We can extend the function $|D f|$ defined in (1.1) to all Borel sets via Carathéodory construction:

$$
|D f|(B):=\inf \{|D f|(\Omega) \mid \Omega \subset \mathrm{X} \text { open, } B \subset \Omega\} \quad \text { for every } B \subset \mathrm{X} \text { Borel. }
$$

This way we obtain a finite Borel measure $|D f|$ on $\mathrm{X}$, which is called the total variation measure of $f$.

Proposition 1.2 (Basic properties of $\mathrm{BV}$ functions) Let $(\mathrm{X}, d, \mathfrak{m})$ be a metric measure space. Let $f, g \in L_{\mathrm{loc}}^{1}(\mathfrak{m})$. Let $B \subset \mathrm{X}$ be Borel and $\Omega \subset \mathrm{X}$ open. Then the following properties hold:

(i) LOWER SEMICONTINUITY. The function $|D \cdot|(\Omega)$ is lower semicontinuous with respect to the $L_{\text {loc }}^{1}\left(\mathfrak{m}_{\llcorner\Omega}\right)$-topology: namely, given any sequence $\left(f_{n}\right)_{n} \subset L_{\text {loc }}^{1}(\mathfrak{m})$ such that $f_{n} \rightarrow f$ in the $L_{\text {loc }}^{1}\left(\mathfrak{m}\llcorner\Omega)\right.$-topology, it holds $|D f|(\Omega) \leq \underline{\lim }_{n}\left|D f_{n}\right|(\Omega)$.

(ii) SubadDitivity. It holds that $|D(f+g)|(B) \leq|D f|(B)+|D g|(B)$.

(iii) COMPACTNESS. Let $\left(f_{n}\right)_{n} \subset L_{\mathrm{loc}}^{1}(\mathfrak{m})$ be a sequence satisfying $\sup _{n}\left|D f_{n}\right|(\mathrm{X})<+\infty$. Then there exist a subsequence $\left(n_{i}\right)_{i}$ and some $f_{\infty} \in L_{\mathrm{loc}}^{1}(\mathfrak{m})$ such that $f_{n_{i}} \rightarrow f_{\infty}$ in $L_{\text {loc }}^{1}(\mathfrak{m})$.

It follows from item (i) of Proposition 1.2 that the space BV(X) is a Borel subset of $L^{1}(\mathfrak{m})$.

Remark 1.3 Let $(\mathrm{X}, d, \mathfrak{m})$ be a metric measure space. Fix $f \in \mathrm{BV}(\mathrm{X})$ and $m>0$. Then

$$
f \wedge m \in \mathrm{BV}(\mathrm{X}) \quad \text { and } \quad|D(f \wedge m)|(\mathrm{X}) \leq|D f|(\mathrm{X}) .
$$

Indeed, pick any $\left(f_{n}\right)_{n} \subset \operatorname{LIP}_{\text {loc }}(\mathrm{X})$ such that $f_{n} \rightarrow f$ in $L_{\text {loc }}^{1}(\mathfrak{m})$ and $\int \operatorname{lip}\left(f_{n}\right) \mathrm{dm} \rightarrow$ $|D f|(\mathrm{X})$. Therefore, it holds that the sequence $\left(f_{n} \wedge m\right)_{n} \subset \mathrm{LIP}_{\text {loc }}(\mathrm{X})$ satisfies $f_{n} \wedge m \rightarrow$ $f \wedge m$ in $L_{\text {loc }}^{1}(\mathfrak{m})$ and $\operatorname{lip}\left(f_{n} \wedge m\right) \leq \operatorname{lip}\left(f_{n}\right)$ for all $n \in \mathbb{N}$. We thus conclude that

$$
|D(f \wedge m)|(\mathrm{X}) \leq \varliminf_{n \rightarrow \infty} \int \operatorname{lip}\left(f_{n} \wedge m\right) \mathrm{dm} \leq \underline{\lim }_{n \rightarrow \infty} \int \operatorname{lip}\left(f_{n}\right) \mathrm{dm}=|D f|(\mathrm{X}),
$$

which yields the statement.

We conclude this subsection by briefly recalling an alternative (but equivalent) approach to the theory of BV functions on abstract metric measure spaces, which has been proposed in $[18,19]$.

A derivation over a metric measure space $(\mathrm{X}, d, \mathfrak{m})$ is a linear map $\boldsymbol{b}: \operatorname{LIP}_{\mathrm{bs}}(\mathrm{X}) \rightarrow L^{0}(\mathfrak{m})$ such that the following properties are satisfied:

(i) LEIBNIZ RULE. $\boldsymbol{b}(f g)=\boldsymbol{b}(f) g+f \boldsymbol{b}(g)$ for every $f, g \in \operatorname{LIP}_{\mathrm{bs}}(\mathrm{X})$. 
(ii) WEAK LOCALITY. There exists a non-negative function $G \in L^{0}(\mathfrak{m})$ such that

$$
|\boldsymbol{b}(f)| \leq G \operatorname{lip}_{\mathrm{a}}(f) \quad \mathfrak{m} \text {-a.e. for every } f \in \operatorname{LIP}_{\mathrm{bs}}(\mathrm{X}) .
$$

The least function $G$ (in the $\mathfrak{m}$-a.e. sense) having this property is denoted by $|\boldsymbol{b}|$.

The space of all derivations over $(\mathrm{X}, d, \mathfrak{m})$ is denoted by $\operatorname{Der}(\mathrm{X})$. The support $\operatorname{spt}(\boldsymbol{b}) \subset \mathrm{X}$ of a derivation $\boldsymbol{b} \in \operatorname{Der}(\mathrm{X})$ is defined as the essential closure of the set $\{|\boldsymbol{b}| \neq 0\}$. Given any $\boldsymbol{b} \in \operatorname{Der}(\mathrm{X})$ with $|\boldsymbol{b}| \in L_{\text {loc }}^{1}(\mathfrak{m})$, we say that $\operatorname{div}(\boldsymbol{b}) \in L^{p}$ for some $p \in[1, \infty]$ provided there exists a (necessarily unique) function $\operatorname{div}(\boldsymbol{b}) \in L^{p}(\mathfrak{m})$ such that $\int \boldsymbol{b}(f) \mathrm{dm}=$ $-\int f \operatorname{div}(\boldsymbol{b}) \mathrm{dm}$ for every $f \in \operatorname{LIP}_{\mathrm{bs}}(\mathrm{X})$. The space of all derivations $\boldsymbol{b} \in \operatorname{Der}(\mathrm{X})$ with $|\boldsymbol{b}| \in L^{\infty}(\mathfrak{m})$ and $\operatorname{div}(\boldsymbol{b}) \in L^{\infty}$ is denoted by $\operatorname{Der}_{\mathrm{b}}(\mathrm{X})$.

Theorem 1.4 (Representation formula for $|D f|$ via derivations) Let (X, $d, \mathfrak{m})$ be a metric measure space. Let $f \in \mathrm{BV}(\mathrm{X})$ be given. Then for every open set $\Omega \subset \mathrm{X}$ it holds that

$$
|D f|(\Omega)=\sup \left\{\int_{\Omega} f \operatorname{div}(\boldsymbol{b}) \mathrm{dm}\left|\boldsymbol{b} \in \operatorname{Der}_{\mathrm{b}}(\mathrm{X}),\right| \boldsymbol{b} \mid \leq 1 \mathfrak{m} \text {-a.e., } \operatorname{spt}(\boldsymbol{b}) \Subset \Omega\right\} .
$$

For a proof of the above representation formula, we refer to [18, Theorem 7.3.4].

\subsection{Sets of finite perimeter}

The study of sets of finite perimeter on abstract metric measure spaces has been initiated in [38] (where, differently from here, the term 'Caccioppoli set' is used). In this subsection we report the definition of set of finite perimeter and its basic properties, more precisely the ones that are satisfied on any metric measure space (without any further assumption).

Definition 1.5 (Set of finite perimeter) Let $(\mathrm{X}, d, \mathfrak{m})$ be a metric measure space. Fix any Borel set $E \subset \mathrm{X}$. Let us define

$$
\mathrm{P}(E, B):=\left|D \mathbb{1}_{E}\right|(B) \text { for every Borel set } B \subset \mathrm{X} .
$$

The quantity $\mathrm{P}(E, B)$ is called perimeter of $E$ in $B$. Then the set $E$ has finite perimeter provided

$$
\mathrm{P}(E):=\mathrm{P}(E, \mathrm{X})<+\infty .
$$

The finite Borel measure $\mathrm{P}(E, \cdot)$ on $\mathrm{X}$ is called the perimeter measure associated to $E$.

Remark 1.6 Given a Borel set $E \subset \mathrm{X}$ satisfying $\mathfrak{m}(E)<+\infty$, it holds that $E$ has finite perimeter if and only if $\mathbb{1}_{E} \in \mathrm{BV}(\mathrm{X})$.

Proposition 1.7 (Basic properties of sets of finite perimeter) Let $(\mathrm{X}, d, \mathfrak{m})$ be a metric measure space. Let $E, F \subset \mathrm{X}$ be sets of finite perimeter. Let $B \subset \mathrm{X}$ be Borel and $\Omega \subset \mathrm{X}$ open. Then:

(i) LOCALITY. If $\mathfrak{m}((E \Delta F) \cap \Omega)=0$, then $P(E, \Omega \cap B)=P(F, \Omega \cap B)$. In particular, it holds that $P(E, \cdot)=P(F, \cdot)$ whenever $\mathfrak{m}(E \Delta F)=0$.

(ii) LOWER SEMICONTINUITY. The function $P(\cdot, \Omega)$ is lower semicontinuous with respect to the $L_{\mathrm{loc}}^{1}\left(\mathfrak{m}\llcorner\Omega)\right.$-topology: namely, if $\left(E_{n}\right)_{n}$ is a sequence of Borel subsets of $\Omega$ such that the convergence $\mathbb{1}_{E_{n}} \rightarrow \mathbb{1}_{E}$ holds in $L_{\mathrm{loc}}^{1}(\mathfrak{m}\llcorner\Omega)$ as $n \rightarrow \infty$, then $P(E, \Omega) \leq$ $\underline{\lim }_{n} P\left(E_{n}, \Omega\right)$.

(iii) Subadditivity. It holds that $P(E \cup F, B)+P(E \cap F, B) \leq P(E, B)+P(F, B)$. 
vi) Complementation. It holds that $P(E, B)=P\left(E^{c}, B\right)$.

(v) COMPACTNESS. Let $\left(E_{n}\right)_{n}$ be a sequence of Borel subsets of X with $\sup _{n} P\left(E_{n}\right)<+\infty$. Then there exist a subsequence $\left(n_{i}\right)_{i}$ and a Borel set $E_{\infty} \subset \mathrm{X}$ such that $\mathbb{1}_{E_{n_{i}}} \rightarrow \mathbb{1}_{E_{\infty}}$ in the $L_{\text {loc }}^{1}(\mathfrak{m})$-topology as $i \rightarrow \infty$.

\subsection{Fine properties of sets of finite perimeter in PI spaces}

The first aim of this subsection is to recall the definition of PI space and its main properties; we refer to [31] for a thorough account about this topic. Thereafter, we shall recall the definition of essential boundary and the main properties of sets of finite perimeter in PI spaces-among others, the isoperimetric inequality, the coarea formula, and the Hausdorff representation of the perimeter measure. Finally, we will discuss the class of isotropic PI spaces, which plays a central role in the rest of the paper.

Definition 1.8 (Doubling measure) A metric measure space $(\mathrm{X}, d, \mathfrak{m})$ is said to be doubling provided there exists a constant $C_{D} \geq 1$ such that

$$
\mathfrak{m}\left(B_{2 r}(x)\right) \leq C_{D} \mathfrak{m}\left(B_{r}(x)\right) \quad \text { for every } x \in \mathrm{X} \text { and } r>0 .
$$

The least such constant $C_{D}$ is called the doubling constant of (X, $\left.d, \mathfrak{m}\right)$.

Remark 1.9 Let $(\mathrm{X}, d, \mathfrak{m})$ be a doubling metric measure space. Then $\operatorname{spt}(\mathfrak{m})=\mathrm{X}$. Indeed, it holds that $\mathfrak{m}\left(B_{r}(x)\right)>0$ for every $x \in \mathrm{X}$ and $r>0$, otherwise $\mathfrak{m}$ would be the null measure. Moreover, the metric space $(\mathrm{X}, d)$ is proper (i.e., bounded closed subsets of $\mathrm{X}$ are compact).

Doubling spaces do not have a definite dimension (not even locally), but still are 'finitedimensional' - in a suitable sense. In light of this, it makes sense to consider the codimensionone Hausdorff measure $\mathcal{H}$, defined below via Carathéodory construction, which takes into account the local change of dimension of the underlying space.

Definition 1.10 (Codimension-one Hausdorff measure) Let $(\mathrm{X}, d, \mathfrak{m})$ be a doubling metric measure space. Given any set $E \subset \mathrm{X}$ and any parameter $\delta>0$, we define

$$
\mathcal{H}_{\delta}(E):=\inf \left\{\sum_{i=1}^{\infty} \frac{\mathfrak{m}\left(B_{r_{i}}\left(x_{i}\right)\right)}{2 r_{i}} \mid\left(x_{i}\right)_{i} \subset \mathrm{X},\left(r_{i}\right)_{i} \subset(0, \delta], E \subset \bigcup_{i \in \mathbb{N}} B_{r_{i}}\left(x_{i}\right)\right\} .
$$

Then we define the codimension-one Hausdorff measure $\mathcal{H}$ on $(\mathrm{X}, d, \mathfrak{m})$ as

$$
\mathcal{H}(E):=\lim _{\delta \searrow 0} \mathcal{H}_{\delta}(E) \text { for every set } E \subset \mathrm{X} .
$$

Both $\mathcal{H}_{\delta}$ and $\mathcal{H}$ are Borel regular outer measures on $\mathrm{X}$.

Definition 1.11 (Ahlfors-regularity) Let $(\mathrm{X}, d, \mathfrak{m})$ be a metric measure space. Let $k \geq 1$ be fixed. Then we say that $(\mathrm{X}, d, \mathfrak{m})$ is $k$-Ahlfors-regular if there exist two constants $\tilde{a} \geq a>0$ such that

$$
a r^{k} \leq \mathfrak{m}\left(B_{r}(x)\right) \leq \tilde{a} r^{k} \quad \text { for every } x \in \mathrm{X} \text { and } r \in(0, \operatorname{diam}(\mathrm{X})) .
$$

It can be readily checked that any Ahlfors-regular space $(\mathrm{X}, d, \mathfrak{m})$ is doubling, with $C_{D}=$ $2^{k} \tilde{a} / a$. 
Definition 1.12 (Weak $(1,1)$-Poincaré inequality) A metric measure space $(\mathrm{X}, d, \mathfrak{m})$ is said to satisfy a weak $(1,1)$-Poincaré inequality provided there exist constants $C_{P}>0$ and $\lambda>1$ such that for any function $f \in \operatorname{LIP}_{\text {loc }}(\mathrm{X})$ and any upper gradient $g$ of $f$ it holds that

$$
\int_{B_{r}(x)}\left|f-f_{x, r}\right| \mathrm{d} \mathfrak{m} \leq C_{P} r \int_{B_{\lambda r}(x)} g \mathrm{dm} \quad \text { for every } x \in \mathrm{X} \text { and } r>0,
$$

where $f_{x, r}:=\mathfrak{m}\left(B_{r}(x)\right)^{-1} \int_{B_{r}(x)} f \mathrm{dm}$ stands for the mean value of $f$ in the ball $B_{r}(x)$.

Lemma 1.13 (Poincaré inequality for $\mathrm{BV}$ functions) Let $(\mathrm{X}, d, \mathfrak{m})$ be a proper metric measure space satisfying a weak $(1,1)$-Poincaré inequality. Let $f \in L_{\text {loc }}^{1}(\mathfrak{m})$ be such that $|D f|(\mathrm{X})<$ $+\infty$. Then it holds that

$$
\int_{B_{r}(x)}\left|f-f_{x, r}\right| \mathrm{d} \mathfrak{m} \leq C_{P} r|D f|\left(B_{\lambda r}(x)\right) \quad \text { for every } x \in \mathrm{X} \text { and } r>0,
$$

where the constants $C_{P}$ and $\lambda$ are chosen as in Definition 1.12.

Proof A standard diagonalisation argument provides us with a sequence $\left(f_{n}\right)_{n} \subset \operatorname{LIP}_{\text {loc }}$ $\left(B_{\lambda r}(x)\right)$ such that $f_{n} \rightarrow f$ in $L_{\mathrm{loc}}^{1}\left(\mathfrak{m}\left\llcorner B_{\lambda r}(x)\right)\right.$ and $|D f|\left(B_{\lambda r}(x)\right)=\lim _{n} \int_{B_{\lambda r}(x)} \operatorname{lip}\left(f_{n}\right) \mathrm{dm}$. Given that the local Lipschitz constant $\operatorname{lip}\left(f_{n}\right)$ is an upper gradient of the function $f_{n}$, it holds that

$$
\int_{B_{r}(x)}\left|f_{n}-\left(f_{n}\right)_{x, r}\right| \mathrm{dm} \leq C_{P} r \int_{B_{\lambda r}(x)} \operatorname{lip}\left(f_{n}\right) \mathrm{dm} \quad \text { for every } n \in \mathbb{N} .
$$

Since the closure of $B_{r}(x)$ is a compact subset of $B_{\lambda r}(x)$, we know that $\mathbb{1}_{B_{r}(x)} f \in$ $L^{1}\left(\mathfrak{m}\left\llcorner B_{r}(x)\right)\right.$ and $\mathbb{1}_{B_{r}(x)} f_{n} \rightarrow \mathbb{1}_{B_{r}(x)} f$ in $L^{1}\left(\mathfrak{m}\left\llcorner B_{r}(x)\right)\right.$, so that $\left(f_{n}\right)_{x, r} \rightarrow f_{x, r}$ as $n \rightarrow \infty$. Moreover, for some function $g \in L^{1}\left(\mathfrak{m}_{\llcorner} B_{r}(x)\right)$ we have (up to a not relabelled subsequence) that $\left|f_{n}(y)\right| \leq g(y)$ for every $n \in \mathbb{N}$ and $\mathfrak{m}$-a.e. $y \in B_{r}(x)$. We can further assume that $f_{n}(y) \rightarrow f(y)$ for m-a.e. $y \in B_{r}(x)$. Given that $\left|f_{n}(y)-\left(f_{n}\right)_{x, r}\right| \leq g(y)+g_{x, r}$ for every $n \in \mathbb{N}$ and $\mathfrak{m}$-a.e. $y \in B_{r}(x)$, we deduce (by dominated convergence theorem) that $\int_{B_{r}(x)}\left|f_{n}-\left(f_{n}\right)_{x, r}\right| \mathrm{d} \mathfrak{m} \rightarrow \int_{B_{r}(x)}\left|f-f_{x, r}\right| \mathrm{dm}$ as $n \rightarrow \infty$. Therefore, by letting $n \rightarrow \infty$ in (1.5) we conclude that the claim (1.4) is verified.

For the purposes of this paper, we shall only consider the following notion of PI space (which is strictly more restrictive than the usual one, where a weak $(1, p)$-Poincaré inequality is required for some exponent $p$ that is possibly greater than 1$)$ :

Definition 1.14 (PI space) We say that a metric measure space $(\mathrm{X}, d, \mathfrak{m})$ is a PI space provided it is doubling and satisfies a weak $(1,1)$-Poincaré inequality.

We introduce the concept of essential boundary in a doubling metric measure space and its main features. The discussion is basically taken from [1,2], apart from a few notational discrepancies.

Given a doubling metric measure space $(\mathrm{X}, d, \mathfrak{m})$, a Borel set $E \subset \mathrm{X}$ and a point $x \in \mathrm{X}$, we define the upper density of $E$ at $x$ and the lower density of $E$ at $x$ as

$$
\bar{D}(E, x):=\varlimsup_{r \searrow 0} \frac{\mathfrak{m}\left(E \cap B_{r}(x)\right)}{\mathfrak{m}\left(B_{r}(x)\right)}, \quad \underline{D}(E, x):=\varliminf_{r \searrow 0}^{\lim } \frac{\mathfrak{m}\left(E \cap B_{r}(x)\right)}{\mathfrak{m}\left(B_{r}(x)\right)},
$$

respectively. Whenever upper and lower densities coincide, their common value is called density of $E$ at $x$ and denoted by $D(E, x)$. We define the essential boundary of the set $E$ as

$$
\partial^{e} E:=\left\{x \in \mathrm{X} \mid \bar{D}(E, x)>0, \bar{D}\left(E^{c}, x\right)>0\right\} .
$$


It clearly holds that the essential boundary $\partial^{e} E$ is contained in the topological boundary $\partial E$. Moreover, we define the set $E^{1 / 2} \subset \partial^{e} E$ of points of density $1 / 2$ as

$$
E^{1 / 2}:=\{x \in \mathrm{X} \mid D(E, x)=1 / 2\} .
$$

Finally, we define the essential interior $E^{1}$ of $E$ as

$$
E^{1}:=\{x \in \mathrm{X} \mid D(E, x)=1\} .
$$

Clearly, it holds that $\partial^{e} E \cap E^{1}=\emptyset$ : if $x \in \partial^{e} E$ then $\underline{D}(E, x)=1-\bar{D}\left(E^{c}, x\right)<1$, so $x \notin E^{1}$.

Remark 1.15 Let $F \subset E \subset X$ be given. Then

$$
\partial^{e} F \subset \partial^{e} E \cup E^{1} .
$$

Indeed, fix any $x \in \partial^{e} F \backslash \partial^{e} E$. Then $\bar{D}(E, x) \geq \bar{D}(F, x)>0$, thus accordingly $\bar{D}\left(E^{c}, x\right)=$ 0 . This forces $D(E, x)=1-D\left(E^{c}, x\right)=1$, so that $x \in E^{1}$. Hence, the claim (1.6) is proven.

The following result is well-known. We report here its full proof for the reader's convenience.

Proposition 1.16 (Properties of the essential boundary) Let $(\mathrm{X}, d, \mathfrak{m})$ be a doubling metric measure space. Let $E, F \subset \mathrm{X}$ be sets of finite perimeter. Then the following properties hold:

(i) It holds that $\partial^{e} E=\partial^{e} E^{c}$.

(ii) We have that

$$
\partial^{e}(E \cup F) \cup \partial^{e}(E \cap F) \subset \partial^{e} E \cup \partial^{e} F .
$$

(iii) If $\mathfrak{m}(E \cap F)=0$, then $\partial^{e} E \subset \partial^{e} F \cup \partial^{e}(E \cup F)$.

(iv) If $\mathfrak{m}(E \cap F)=0$, then $\partial^{e} E \cup \partial^{e} F=\partial^{e}(E \cup F) \cup\left(\partial^{e} E \cap \partial^{e} F\right)$.

Proof (i) It trivially stems from the very definition of essential boundary.

(ii) First of all, fix $x \in \partial^{e}(E \cup F)$. Note that $\bar{D}(E \cup F, x) \leq \bar{D}(E, x)+\bar{D}(F, x)$, as it follows from

$$
\begin{aligned}
\bar{D}(E \cup F, x) & =\varlimsup_{r \searrow 0} \frac{\mathfrak{m}\left((E \cup F) \cap B_{r}(x)\right)}{\mathfrak{m}\left(B_{r}(x)\right)} \leq \varlimsup_{r \searrow 0}\left[\frac{\mathfrak{m}\left(E \cap B_{r}(x)\right)}{\mathfrak{m}\left(B_{r}(x)\right)}+\frac{\mathfrak{m}\left(F \cap B_{r}(x)\right)}{\mathfrak{m}\left(B_{r}(x)\right)}\right] \\
& \leq \varlimsup_{r \searrow 0} \frac{\mathfrak{m}\left(E \cap B_{r}(x)\right)}{\mathfrak{m}\left(B_{r}(x)\right)}+\varlimsup_{r \searrow 0} \frac{\mathfrak{m}\left(F \cap B_{r}(x)\right)}{\mathfrak{m}\left(B_{r}(x)\right)}=\bar{D}(E, x)+\bar{D}(F, x) .
\end{aligned}
$$

Therefore, the fact that $\bar{D}(E \cup F, x)>0$ implies either $\bar{D}(E, x)>0$ or $\bar{D}(F, x)>0$. Furthermore, we have that $\bar{D}\left(E^{c}, x\right), \bar{D}\left(F^{c}, x\right) \geq \bar{D}\left(E^{c} \cap F^{c}, x\right)=\bar{D}\left((E \cup F)^{c}, x\right)>$ 0 , whence $x \in \partial^{e} E \cup \partial^{e} F$.

In order to prove that even the inclusion $\partial^{e}(E \cap F) \subset \partial^{e} E \cup \partial^{e} F$ is verified, it is just sufficient to combine the previous case with item (i):

$$
\partial^{e}(E \cap F)=\partial^{e}(E \cap F)^{c}=\partial^{e}\left(E^{c} \cup F^{c}\right) \subset \partial^{e} E^{c} \cup \partial^{e} F^{c}=\partial^{e} E \cup \partial^{e} F .
$$

Hence, the proof of (1.7) is complete. 
(iii) Pick any point $x \in \partial^{e} E$. First of all, notice that $\bar{D}(E \cup F, x), \bar{D}\left(F^{c}, x\right) \geq \bar{D}(E, x)>0$. Moreover, it holds that

$$
\begin{aligned}
\bar{D}\left((E \cup F)^{c}, x\right)+\bar{D}(F, x) & =\varlimsup_{r \searrow 0} \frac{\mathfrak{m}\left(E^{c} \cap F^{c} \cap B_{r}(x)\right)}{\mathfrak{m}\left(B_{r}(x)\right)}+\varlimsup_{r \searrow 0} \frac{\mathfrak{m}\left(F \cap B_{r}(x)\right)}{\mathfrak{m}\left(B_{r}(x)\right)} \\
& \geq \varlimsup_{r \searrow 0}\left[\frac{\mathfrak{m}\left(E^{c} \cap F^{c} \cap B_{r}(x)\right)}{\mathfrak{m}\left(B_{r}(x)\right)}+\frac{\mathfrak{m}\left(F \cap B_{r}(x)\right)}{\mathfrak{m}\left(B_{r}(x)\right)}\right] \\
& =\varlimsup_{r \searrow 0} \frac{\mathfrak{m}\left(E^{c} \cap B_{r}(x)\right)}{\mathfrak{m}\left(B_{r}(x)\right)}=\bar{D}\left(E^{c}, x\right)>0,
\end{aligned}
$$

whence either $\bar{D}\left((E \cup F)^{c}, x\right)>0$ or $\bar{D}(F, x)>0$. This shows that $x \in \partial^{e} F \cup \partial^{e}(E \cup F)$. (iv) Item (ii) grants that $\partial^{e}(E \cup F) \cup\left(\partial^{e} E \cap \partial^{e} F\right) \subset \partial^{e} E \cup \partial^{e} F$. Conversely, item (iii) yields

$\partial^{e} E \cup \partial^{e} F \subset\left(\partial^{e}(E \cup F) \cup \partial^{e} E\right) \cap\left(\partial^{e}(E \cup F) \cup \partial^{e} F\right)=\partial^{e}(E \cup F) \cup\left(\partial^{e} E \cap \partial^{e} F\right)$,

thus obtaining the identity $\partial^{e} E \cup \partial^{e} F=\partial^{e}(E \cup F) \cup\left(\partial^{e} E \cap \partial^{e} F\right)$.

In the setting of PI spaces, functions of bounded variation and sets of finite perimeters present several fine properties, as we are going to describe.

Theorem 1.17 (Relative isoperimetric inequality on PI spaces [38]) Let (X, $d, \mathfrak{m})$ be a PI space. Then there exists a constant $C_{I}>0$ such that the relative isoperimetric inequality is satisfied: given any set $E \subset \mathrm{X}$ of finite perimeter, it holds that

$$
\min \left\{\mathfrak{m}\left(E \cap B_{r}(x)\right), \mathfrak{m}\left(E^{c} \cap B_{r}(x)\right)\right\} \leq C_{I}\left(\frac{r^{s}}{\mathfrak{m}\left(B_{r}(x)\right)}\right)^{1 / s-1} P\left(E, B_{2 \lambda r}(x)\right)^{s / s-1}
$$

for every $x \in \mathrm{X}$ and $r>0$, where $s>1$ is any exponent greater than $\log _{2}\left(C_{D}\right)$.

As an immediate consequence of Theorem 1.17, we have that a given set of finite perimeter $E$ in a PI space $(\mathrm{X}, d, \mathfrak{m})$ has null perimeter if and only if either $\mathfrak{m}(E)=0$ or $\mathfrak{m}\left(E^{c}\right)=0$.

Theorem 1.18 (Global isoperimetric inequality on Ahlfors regular PI spaces) Let (X, $d, \mathfrak{m})$ be a $k$-Ahlfors regular PI space, with $k>1$. Then there exists a constant $C_{I}^{\prime}>0$ such that $\min \left\{\mathfrak{m}(E), \mathfrak{m}\left(E^{c}\right)\right\} \leq C_{I}^{\prime} P(E)^{k / k-1} \quad$ for every set $E \subset \mathrm{X}$ of finite perimeter.

Proof As proven in [38], there exists a constant $C_{I}^{\prime}>0$ such that

$$
\min \left\{\mathfrak{m}\left(E \cap B_{r}(x)\right), \mathfrak{m}\left(E^{c} \cap B_{r}(x)\right)\right\} \leq C_{I}^{\prime} \mathrm{P}\left(E, B_{2 \lambda r}(x)\right)^{k / k-1}
$$

for every $x \in \mathrm{X}$ and $r>0$. By letting $r \rightarrow+\infty$ in (1.10), we conclude that (1.9) is satisfied.

Theorem 1.19 (Coarea formula [38]) Let $(\mathrm{X}, d, \mathfrak{m})$ be a PI space. Fix $f \in L_{\mathrm{loc}}^{1}(\mathfrak{m})$ and an open set $\Omega \subset \mathrm{X}$. Then the function $\mathbb{R} \ni t \mapsto P(\{f>t\}, \Omega) \in[0,+\infty]$ is Borel measurable and it holds

$$
|D f|(\Omega)=\int_{-\infty}^{+\infty} P(\{f>t\}, \Omega) \mathrm{d} t .
$$

In particular, if $f \in \mathrm{BV}(\mathrm{X})$, then $\{f>t\}$ has finite perimeter for a.e. $t \in \mathbb{R}$. 
Remark 1.20 Given a PI space (X, $d, \mathfrak{m})$ and any point $x \in \mathrm{X}$, it holds that the set $B_{r}(x)$ has finite perimeter for a.e. radius $r>0$. This fact follows from the coarea formula (by applying it to the distance function from $x)$. Furthermore, it also holds that $\mathcal{H}\left(\partial B_{r}(x)\right)<+\infty$ for a.e. $r>0$, as a consequence of [2, Proposition 5.1].

A function $f \in \mathrm{BV}(\mathrm{X})$ is said to be simple provided it can be written as $f=\sum_{i=1}^{n} \lambda_{i} \mathbb{1}_{E_{i}}$, for some $\lambda_{1}, \ldots, \lambda_{n} \in \mathbb{R}$ and some sets of finite perimeter $E_{1}, \ldots, E_{n} \subset \mathrm{X}$ having finite $\mathfrak{m}$-measure. It holds that any function of bounded variation in a PI space can be approximated by a sequence of simple BV functions (with a uniformly bounded total variation), as we are going to state in the next well-known result. Nevertheless, we recall the proof of this fact for the sake of completeness.

Lemma 1.21 (Density of simple BV functions) Let $(\mathrm{X}, d, \mathfrak{m})$ be a PI space and $K \subset \mathrm{X} a$ compact set. Fix any $f \in \mathrm{BV}(\mathrm{X})$ with $\operatorname{spt}(f) \subset K$. Then there exists a sequence $\left(f_{n}\right)_{n} \subset$ $\mathrm{BV}(\mathrm{X})$ of simple functions with $\operatorname{spt}\left(f_{n}\right) \subset K$ such that $f_{n} \rightarrow f$ in $L^{1}(\mathfrak{m})$ and $\left|D f_{n}\right|(\mathrm{X}) \leq$ $|D f|(\mathrm{X})$ for all $n \in \mathbb{N}$.

Proof Given that $f^{m}:=(f \wedge m) \vee(-m) \rightarrow f$ in $L^{1}(\mathfrak{m})$ as $m \rightarrow \infty$ and $\left|D f^{m}\right|(\mathrm{X}) \leq$ $|D f|(\mathrm{X})$ for all $m>0$ by Remark 1.3, it suffices to prove the statement under the additional assumption that the function $f$ is essentially bounded, say that $-k<f<k$ holds $\mathfrak{m}$-a.e. for some $k \in \mathbb{N}$. Let us fix any $n \in \mathbb{N}$. Given any $i=-k n+1, \ldots, k n$, we can choose $t_{i, n} \in((i-1) / n, i / n)$ such that

$$
\frac{\mathrm{P}\left(\left\{f>t_{i, n}\right\}\right)}{n} \leq \int_{(i-1) / n}^{i / n} \mathrm{P}(\{f>t\}) \mathrm{d} t .
$$

Then we define the simple BV function $f_{n}$ on $\mathrm{X}$ as

$$
f_{n}:=-k+\frac{1}{n} \sum_{i=-k n+1}^{k n} \mathbb{1}_{\left\{f>t_{i, n}\right\}} .
$$

It can be readily checked that $\left|D f_{n}\right|(\mathrm{X}) \leq|D f|(\mathrm{X})$. Indeed, notice that

$$
\left|D f_{n}\right|(\mathrm{X}) \leq \frac{1}{n} \sum_{i=-k n+1}^{k n} \mathrm{P}\left(\left\{f>t_{i, n}\right\}\right) \stackrel{(1.12)}{\leq} \int_{-k}^{k} \mathrm{P}(\{f>t\}) \mathrm{d} t \stackrel{(1.11)}{=}|D f|(\mathrm{X}) .
$$

Furthermore, let us define $E_{i, n}:=\left\{t_{i, n}<f \leq t_{i+1, n}\right\}$ for every $i=-k n+1, \ldots, k n-1$. Moreover, we set $E_{-k n, n}:=\left\{-k<f \leq t_{-k n+1, n}\right\}$ and $E_{k n, n}:=\left\{t_{k n, n}<f<k\right\}$. Therefore, it holds that

$$
\begin{aligned}
f_{n} & =-k+\frac{1}{n} \sum_{i=-k n+1}^{k n} \sum_{j=i}^{k n} \mathbb{1}_{E_{j, n}}=-k+\frac{1}{n} \sum_{i=-k n+1}^{k n}(i+k n) \mathbb{1}_{E_{i, n}} \\
& =-k \mathbb{1}_{E_{-k n, n}}+\sum_{i=-k n+1}^{k n} \frac{i}{n} \mathbb{1}_{E_{i, n}},
\end{aligned}
$$

thus accordingly $\left|f-f_{n}\right|=|f-i / n| \leq 1 / n$ on $E_{i, n}$ for all $i=-k n, \ldots, k n$. This ensures that

$$
\int\left|f-f_{n}\right| \mathrm{d} \mathfrak{m}=\sum_{i=-k n}^{k n} \int_{E_{i, n}}|f-i / n| \mathrm{d} \mathfrak{m} \leq \frac{\mathfrak{m}(K)}{n} \stackrel{n}{\longrightarrow} 0 .
$$


Therefore, we have that $f_{n} \rightarrow f$ in $L^{1}(\mathfrak{m})$. Since $\operatorname{spt}\left(f_{n}\right) \subset K$ for every $n \in \mathbb{N}$ by construction, the proof of the statement is achieved.

Remark 1.22 In the proof of Lemma 1.21 we obtained a stronger property: each approximating function $f_{n}$ (say, $f_{n}=\sum_{i=1}^{k_{n}} \lambda_{i}^{n} \mathbb{1}_{E_{i}^{n}}$ ) can be required to satisfy $\sum_{i=1}^{k_{n}}\left|\lambda_{i}^{n}\right| \mathrm{P}\left(E_{i}^{n}\right) \leq$ $|D f|(\mathrm{X})$.

The following result states that, in the context of PI spaces, the perimeter measure admits an integral representation (with respect to the codimension-one Hausdorff measure):

Theorem 1.23 (Representation of the perimeter measure) Let $(\mathrm{X}, d, \mathfrak{m})$ be a PI space. Let $E \subset \mathrm{X}$ be a set of finite perimeter. Then the perimeter measure $P(E, \cdot)$ is concentrated on the Borel set

$$
\Sigma_{\tau}(E):=\left\{x \in \mathrm{X} \mid \frac{\lim }{r \searrow 0} \min \left\{\frac{\mathfrak{m}\left(E \cap B_{r}(x)\right)}{\mathfrak{m}\left(B_{r}(x)\right)}, \frac{\mathfrak{m}\left(E^{c} \cap B_{r}(x)\right)}{\mathfrak{m}\left(B_{r}(x)\right)}\right\} \geq \tau\right\} \subset \partial^{e} E,
$$

where $\tau \in(0,1 / 2)$ is a constant depending just on $C_{D}, C_{P}$ and $\lambda$. Moreover, the set $\partial^{e} E \backslash \Sigma_{\tau}(E)$ is $\mathcal{H}$-negligible and it holds that $\mathcal{H}\left(\partial^{e} E\right)<+\infty$. Finally, there exist a constant $\gamma>0$ (depending on $C_{D}, C_{P}, \lambda$ ) and a Borel function $\theta_{E}: \partial^{e} E \rightarrow\left[\gamma, C_{D}\right]$ such that $P(E, \cdot)=\theta_{E} \mathcal{H}\left\llcorner\partial^{e} E\right.$, namely

$$
P(E, B)=\int_{B \cap \partial^{e} E} \theta_{E} \mathrm{~d} \mathcal{H} \text { for every Borel set } B \subset \mathrm{X} .
$$

We shall sometimes consider $\theta_{E}$ as a Borel function defined on the whole space $\mathrm{X}$, by declaring that $\theta_{E}:=0$ on the set $\mathrm{X} \backslash \partial^{e} E$.

Proof The result is mostly proven in [2, Theorem 5.3]. The fact that the measure $\mathrm{P}(E, \cdot)$ is concentrated on the set $\Sigma_{\tau}(E)$ is shown in [2, Theorem 5.4]. Finally, the upper bound $\theta_{E} \leq C_{D}$ has been obtained in [7, Theorem 4.6].

Lemma 1.24 Let $(\mathrm{X}, d, \mathfrak{m})$ be a PI space. Let $F \subset E \subset \mathrm{X}$ be two sets of finite perimeter such that $P(E)=P(F)+P(E \backslash F)$. Then $\mathcal{H}\left(\partial^{e} F \backslash \partial^{e} E\right)=0$.

Proof By using item (iii) of Proposition 1.7 we deduce that

$$
\mathrm{P}(E)=\mathrm{P}\left(E, \partial^{e} E\right) \leq \mathrm{P}\left(F, \partial^{e} E\right)+\mathrm{P}\left(E \backslash F, \partial^{e} E\right) \leq \mathrm{P}(F)+\mathrm{P}(E \backslash F)=\mathrm{P}(E),
$$

which forces the identity $\mathrm{P}\left(F, \partial^{e} E\right)=\mathrm{P}(F)$. This implies $\left(\theta_{F} \mathcal{H}\right)\left(\partial^{e} F \backslash \partial^{e} E\right)=$ $\mathrm{P}\left(F,\left(\partial^{e} E\right)^{c}\right)=0$ by Theorem 1.23 , whence accordingly $\mathcal{H}\left(\partial^{e} F \backslash \partial^{e} E\right)=0$, as required.

The density function $\theta_{E}$ that appears in the Hausdorff representation formula for $\mathrm{P}(E, \cdot)$ might depend on the set $E$ itself (cf. Example 1.27 below for an instance of this phenomenon). On the other hand, the new results that we are going to present in this paper require the density $\theta_{E}$ to be 'universal' - in a suitable sense. The precise formulation of this property is given in the next definition, which has been proposed in [7, Definition 6.1].

Definition 1.25 (Isotropic space) Let $(\mathrm{X}, d, \mathfrak{m})$ be a PI space. Then we say that $(\mathrm{X}, d, \mathfrak{m})$ is isotropic provided for any pair of sets $E, F \subset \mathrm{X}$ of finite perimeter satisfying $F \subset E$ it holds that

$$
\theta_{F}(x)=\theta_{E}(x) \text { for } \mathcal{H} \text {-a.e. } x \in \partial^{e} F \cap \partial^{e} E \text {. }
$$


In order to provide examples and counterexamples, it will be convenient to consider the metric measure space we are going to construct. Given any $n \in \mathbb{N}$, we define the $n$-spider $S_{n}$ as

$$
S_{n}:=\{o\} \sqcup\left(R_{1} \cup \cdots \cup R_{n}\right), \quad \text { where } R_{i}:=\{i\} \times(0,+\infty) \text { for every } i=1, \ldots, n .
$$

We say that $o$ is the origin of $S_{n}$, while $R_{1}, \ldots, R_{n}$ are the rays of $S_{n}$. We identify $o$ with $(i, 0)$ for every $i=1, \ldots, n$. It holds that $\left(S_{n}, d, \mathfrak{m}\right)$ is an Ahlfors-regular PI space, where $d$ is given by

$$
d((i, t),(j, s)):= \begin{cases}|t-s| & \text { if } \quad i=j, \\ t+s & \text { if } i \neq j\end{cases}
$$

and $\mathfrak{m}$ stands for the 1-dimensional Hausdorff measure on $\left(S_{n}, d\right)$.

Lemma 1.26 Let $E$ be a set of finite perimeter in the $n$-spider $\left(S_{n}, d, \mathfrak{m}\right)$. Let o and $R_{1}, \ldots, R_{n}$ be the origin and the rays of $S_{n}$, respectively. Then the essential boundary $\partial^{e} E$ is a finite set and each intersection $E \cap R_{i}$ is $\mathfrak{m}$-a.e. equivalent to the union of finitely many subintervals of $R_{i}$.

Moreover, calling I the family of all $i \in\{1, \ldots, n\}$ such that $E \cap R_{i}$ contains (up to $m$-null sets) a set of the form $\{i\} \times(0, \varepsilon)$ for some $\varepsilon>0$, and $k \in\{1, \ldots, n\}$ the cardinality of $I$, it holds that

$$
P(E, \cdot)=\lambda \delta_{o}+\sum_{p \in \partial^{e} E \backslash\{o\}} \delta_{p}, \quad \text { where } \lambda:=\min \{k, n-k\} .
$$

Proof Possibly replacing $E$ with its complement $E^{c}$-an operation which does not affect $\mathrm{P}(E, \cdot)$ nor $\partial^{e} E$-we can suppose without loss of generality that $k \leq n-k$, thus $\lambda=k$. The first statement readily follows from the fact that $\mathrm{P}(E, \cdot)$ is absolutely continuous with respect to the counting measure on $S_{n}$. Indeed, the Ahlfors-regularity of $\left(S_{n}, d, \mathfrak{m}\right)$ grants that $\mathcal{H}$ is equivalent to the 0 -dimensional Hausdorff measure, whence Theorem 1.23 yields the previous claim. To prove the last statement, first observe that-since each ray $R_{i}$ can be identified with $(0,+\infty)$-it holds

$$
\mathrm{P}(E, \cdot)\left\llcorner R_{i}=\sum_{p \in \partial^{e} E \cap R_{i}} \delta_{p} \quad \text { for every } i=1, \ldots, n .\right.
$$

It thus remains to characterise $\mathrm{P}(E, \cdot)\llcorner\Omega$ for some open neighbourhood $\Omega$ of $o$. To this aim, take any $r \in(0,1 / 2)$ such that $\{i\} \times(0, r)$ is $\mathfrak{m}$-a.e. contained in $E$ for every $i \in I$ and call $\Omega:=B_{r}(o)$. It is then clear by construction that $\partial^{e} E \cap \Omega \subset\{o\}$. Therefore, in order to prove (1.17) it just suffices to show that $\mathrm{P}(E, \Omega)=k$. On the one hand, we define the sequence $\left(g_{j}\right)_{j \geq 3} \subset \operatorname{LIP}(\Omega)$ as

$$
g_{j}(x):= \begin{cases}\min \{j t, 1\} & \text { if } \quad x \in \Omega \cap R_{i} \text { for some } i \in I \text { and } x=(i, t), \\ 0 & \text { if } \quad x \in \Omega \backslash \bigcup_{i \in I} R_{i} .\end{cases}
$$

Hence, it holds that $g_{j} \rightarrow \mathbb{1}_{E}$ in $L^{1}\left(\mathfrak{m}\llcorner\Omega)\right.$ and $\int_{\Omega} \operatorname{lip}\left(g_{j}\right) \mathrm{dm}=k$ for all $j \geq 3$, thus $\mathrm{P}(E, \Omega) \leq k$.

On the other hand, fix any sequence $\left(f_{j}\right)_{j} \subseteq \operatorname{LIP}_{\text {loc }}(\Omega)$ satisfying $f_{j} \rightarrow \mathbb{1}_{E}$ in $L_{\text {loc }}^{1}(\mathfrak{m}\llcorner\Omega)$

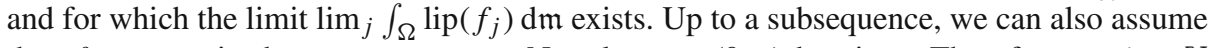
that $f_{j} \rightarrow \mathbb{1}_{E}$ in the $\mathfrak{m}\llcorner\Omega$-a.e. sense. Now let $\varepsilon \in(0, r)$ be given. Then for any $j \in \mathbb{N}$ sufficiently large we can find points $x_{i} \in B_{\varepsilon}(o) \cap R_{i}$ with $i \in\{1, \ldots, n\}$, such that $\mid f_{j}\left(x_{i}\right)-$ 
$1 \mid \leq \varepsilon$ for all $i \in I$ and $\left|f_{j}\left(x_{i}\right)\right| \leq \varepsilon$ for all $i \notin I$. Given that $\left|\mathbb{1}_{I}(i)-c\right| \leq\left|f_{j}\left(x_{i}\right)-c\right|+\varepsilon$ for all $i \in\{1, \ldots, n\}$ and $c \in \mathbb{R}$, we see that

$$
\begin{aligned}
k & =\min _{c \in \mathbb{R}} \sum_{i=1}^{n}\left|\mathbb{1}_{I}(i)-c\right| \leq n \varepsilon+\min _{c \in \mathbb{R}} \sum_{i=1}^{n}\left|f_{j}\left(x_{i}\right)-c\right| \leq n \varepsilon+\sum_{i=1}^{n}\left|f_{j}\left(x_{i}\right)-f_{j}(o)\right| \\
& \leq n \varepsilon+\sum_{i=1}^{n} \int_{\Omega \cap R_{i}} \operatorname{lip}\left(f_{j}\right) \mathrm{d} \mathfrak{m}=n \varepsilon+\int_{\Omega} \operatorname{lip}\left(f_{j}\right) \mathrm{d} \mathfrak{m} .
\end{aligned}
$$

By first letting $j \rightarrow \infty$ and then $\varepsilon \rightarrow 0$, we finally conclude that $\lim _{j} \int \operatorname{lip}\left(f_{j}\right) \mathrm{dm} \geq k$ and thus accordingly $\mathrm{P}(E, \Omega)=k$. Therefore, the proof of the last statement is completed.

Example 1.27 The 4-spider $\left(S_{4}, d, \mathfrak{m}\right)$ is not isotropic: calling $o$ its origin and $R_{1}, R_{2}, R_{3}, R_{4}$ its rays, we know from Lemma 1.26 that $\theta_{R_{1}}(o)=1$ and $\theta_{R_{1} \cup R_{2}}(o)=2$.

We shall also sometimes work with PI spaces satisfying the following property:

Definition 1.28 (Two-sidedness property) Let $(\mathrm{X}, d, \mathfrak{m})$ be a PI space. Then we say that $(\mathrm{X}, d, \mathfrak{m})$ has the two-sidedness property provided it holds that

$$
\mathcal{H}\left(\partial^{e} E \cap \partial^{e} F \cap \partial^{e}(E \cup F)\right)=0 \text { for any disjoint sets } E, F \subset \mathrm{X} \text { of finite perimeter. }
$$

It immediately follows from [7, Proposition 6.2] that every PI space having the two-sidedness property is isotropic, while the converse implication might fail (as shown by the following example).

Example 1.29 The 3-spider $\left(S_{3}, d, \mathfrak{m}\right)$ is an isotropic space: indeed, if $E \subset S_{3}$ is any set of finite perimeter such that $o \in \partial^{e} E$, then $\theta_{E}(o)=1$ by Lemma 1.26. Moreover, the space $\left(S_{3}, d, \mathfrak{m}\right)$ does not have the two-sidedness property, as $\partial^{e} R_{1} \cap \partial^{e} R_{2} \cap \partial^{e}\left(R_{1} \cup R_{2}\right)=\{o\}$ and $\mathcal{H}(\{o\})>0$.

The same arguments show that, given a radius $r>0$, the closure $\mathrm{X}_{r}$ of the ball $B_{r}(o)$ in $S_{3}$ (endowed with the restricted distance $d_{\mathrm{X}_{r} \times \mathrm{X}_{r}}$ and measure $\mathfrak{m}_{\left\llcorner\mathrm{X}_{r}\right.}$ ) is an isotropic, Ahlforsregular PI space which does not have the two-sidedness property.

A sufficient condition for the two-sidedness property to hold is provided by the following result:

Lemma 1.30 Let $(\mathrm{X}, d, \mathfrak{m})$ be a PI space with the following property:

$$
\mathcal{H}\left(\partial^{e} E \backslash E^{1 / 2}\right)=0 \quad \text { for every set } E \subset \mathrm{X} \text { of finite perimeter }
$$

(or, equivalently, the measure $\mathrm{P}(E, \cdot)$ is concentrated on $\left.E^{1 / 2}\right)$. Then the space $(\mathrm{X}, d, \mathfrak{m})$ has the two-sidedness property.

Proof Fix two disjoint sets $E, F \subset \mathrm{X}$ of finite perimeter. Given any $x \in E^{1 / 2} \cap F^{1 / 2}$, we have

$$
D(E \cup F, x)=\lim _{r \searrow 0} \frac{\mathfrak{m}\left(E \cap B_{r}(x)\right)}{\mathfrak{m}\left(B_{r}(x)\right)}+\lim _{r \searrow 0} \frac{\mathfrak{m}\left(F \cap B_{r}(x)\right)}{\mathfrak{m}\left(B_{r}(x)\right)}=D(E, x)+D(F, x)=1,
$$

thus in particular $x \notin(E \cup F)^{1 / 2}$. This shows that $E^{1 / 2} \cap F^{1 / 2} \cap(E \cup F)^{1 / 2}=\emptyset$, whence we can conclude that $\mathcal{H}\left(\partial^{e} E \cap \partial^{e} F \cap \partial^{e}(E \cup F)\right)=0$. This proves the two-sidedness property. 
Example 1.31 (Examples of isotropic spaces) Let us conclude the section by expounding which classes of PI spaces are known to be isotropic (to the best of our knowledge):

(i) Weighted Euclidean spaces (induced by a continuous, strong $A_{\infty}$ weight).

(ii) Carnot groups.

(iii) $R C D(K, N)$ spaces, with $K \in \mathbb{R}$ and $N<\infty$. In particular, all (weighted) Riemannian manifolds whose Ricci curvature is bounded from below.

Isotropicity of the spaces in (i) and (ii) is shown in [7, Section 7] and [8], respectively. Also, it follows from the rectifiability results in [26,27] that all Carnot groups of step 2 satisfy (1.19), so also the two-sidedness property. About item (iii), it follows from the results in $[3,14]$ that all $R C D(K, N)$ spaces satisfy (1.19), whence they have the two-sidedness property (and thus are isotropic).

\section{Decomposability of a set of finite perimeter}

This section is entirely devoted to the decomposability properties of sets of finite perimeter in isotropic PI spaces. An indecomposable set is, roughly speaking, a set of finite perimeter that is connected in a measure-theoretical sense. Section 2.1 consists of a detailed study of the basic properties of this class of sets. In Sect. 2.2 we will prove that any set of finite perimeter can be uniquely expressed as disjoint union of indecomposable sets (cf. Theorem 2.14). The whole discussion is strongly inspired by the results of [4], where the decomposability of sets of finite perimeter in the Euclidean setting has been systematically investigated. Actually, many of the results (and the relative proofs) in this section are basically just a reformulation-in the metric setting - of the corresponding ones in $\mathbb{R}^{n}$, proven in [4]. We postpone to Remark 2.19 the discussion of the main differences between the case of isotropic PI spaces and the Euclidean one.

\subsection{Definition of decomposable set and its basic properties}

Let us begin with the definition of decomposable set and indecomposable set in a general metric measure space.

Definition 2.1 (Decomposable and indecomposable sets) Let $(\mathrm{X}, d, \mathfrak{m})$ be a metric measure space. Let $E \subset \mathrm{X}$ be a set of finite perimeter. Given any Borel set $B \subset \mathrm{X}$, we say that $E$ is decomposable in $B$ provided there exists a partition $\{F, G\}$ of $E \cap B$ into sets of finite perimeter such that $\mathfrak{m}(F), \mathfrak{m}(G)>0$ and $\mathrm{P}(E, B)=\mathrm{P}(F, B)+\mathrm{P}(G, B)$. On the other hand, we say that $E$ is indecomposable in $B$ if it is not decomposable in $B$. For brevity, we say that $E$ is decomposable (resp. indecomposable) provided it is decomposable in $\mathrm{X}$ (resp. indecomposable in $\mathrm{X})$.

Observe that the property of being decomposable/indecomposable is invariant under modifications on $\mathfrak{m}$-null sets and that any $\mathfrak{m}$-negligible set is indecomposable.

Remark 2.2 Let $E \subset \mathrm{X}$ be a set of finite perimeter. Let $\left\{E_{n}\right\}_{n \in \mathbb{N}}$ be a partition of $E$ into sets of finite perimeter and let $\Omega \subset \mathrm{X}$ be any open set. Then it holds that:

$$
\mathrm{P}(E, \Omega)=\sum_{n=0}^{\infty} \mathrm{P}\left(E_{n}, \Omega\right) \Longleftrightarrow \mathrm{P}(E, \Omega) \geq \sum_{n=0}^{\infty} \mathrm{P}\left(E_{n}, \Omega\right) .
$$


Indeed, it can be readily checked that $\mathbb{1}_{\cup_{n \leq N} E_{n}} \rightarrow \mathbb{1}_{E}$ in $L_{\text {loc }}^{1}(\mathfrak{m})$ as $N \rightarrow \infty$, whence items (ii) and (iii) of Proposition 1.7 grant that the inequality

$$
\mathrm{P}(E, \Omega) \leq \varliminf_{N \rightarrow \infty} \mathrm{P}\left(\bigcup_{n \leq N} E_{n}, \Omega\right) \leq \lim _{N \rightarrow \infty} \sum_{n=0}^{N} \mathrm{P}\left(E_{n}, \Omega\right)=\sum_{n=0}^{\infty} \mathrm{P}\left(E_{n}, \Omega\right)
$$

is always verified.

Lemma 2.3 Let $(\mathrm{X}, d, \mathfrak{m})$ be an isotropic PI space. Let $E, F \subset \mathrm{X}$ be sets of finite perimeter and let $B \subset \mathrm{X}$ be any Borel set. Then the following implications hold:

(i) If $P(E \cup F, B)=P(E, B)+P(F, B)$, then $\mathcal{H}\left(\partial^{e} E \cap \partial^{e} F \cap B\right)=0$.

(ii) If $\mathfrak{m}(E \cap F)=0$ and $\mathcal{H}\left(\partial^{e} E \cap \partial^{e} F \cap B\right)=0$, then $P(E \cup F, B)=P(E, B)+P(F, B)$.

Proof (i) Suppose that $\mathrm{P}(E \cup F, B)=\mathrm{P}(E, B)+\mathrm{P}(F, B)$. A trivial set-theoretic argument yields

$$
\begin{aligned}
& \left(\theta_{E \cup F} \mathcal{H}\right)\left(\left(\partial^{e} E \cup \partial^{e} F\right) \cap B\right) \\
& =\left(\theta_{E \cup F} \mathcal{H}\right)\left(\left(\partial^{e} E \backslash \partial^{e} F\right) \cap B\right)+\left(\theta_{E \cup F} \mathcal{H}\right)\left(\left(\partial^{e} F \backslash \partial^{e} E\right) \cap B\right) \\
& \quad+\left(\theta_{E \cup F} \mathcal{H}\right)\left(\partial^{e} E \cap \partial^{e} F \cap B\right) \\
& \quad=\left(\theta_{E \cup F} \mathcal{H}\right)\left(\partial^{e} E \cap B\right)+\left(\theta_{E \cup F} \mathcal{H}\right)\left(\partial^{e} F \cap B\right)-\left(\theta_{E \cup F} \mathcal{H}\right)\left(\partial^{e} E \cap \partial^{e} F \cap B\right) .
\end{aligned}
$$

Given that $\theta_{E \cup F}$ is assumed to be null on the complement of $\partial^{e}(E \cup F)$, we deduce that

$$
\begin{aligned}
& \left(\theta_{E \cup F} \mathcal{H}\right)\left(\partial^{e} E \cap B\right) \\
& \quad=\left(\theta_{E \cup F} \mathcal{H}\right)\left(\partial^{e} E \cap \partial^{e}(E \cup F) \cap B\right) \stackrel{(1.15)}{=}\left(\theta_{E} \mathcal{H}\right)\left(\partial^{e} E \cap \partial^{e}(E \cup F) \cap B\right), \\
& \left(\theta_{E \cup F} \mathcal{H}\right)\left(\partial^{e} F \cap B\right) \\
& \quad=\left(\theta_{E \cup F} \mathcal{H}\right)\left(\partial^{e} F \cap \partial^{e}(E \cup F) \cap B\right) \stackrel{(1.15)}{=}\left(\theta_{F} \mathcal{H}\right)\left(\partial^{e} F \cap \partial^{e}(E \cup F) \cap B\right) .
\end{aligned}
$$

Accordingly, it holds that

$$
\begin{aligned}
\mathrm{P}(E \cup F, B) & \stackrel{(1.14)}{=}\left(\theta_{E \cup F} \mathcal{H}\right)\left(\partial^{e}(E \cup F) \cap B\right) \stackrel{(1.7)}{\leq}\left(\theta_{E \cup F} \mathcal{H}\right)\left(\left(\partial^{e} E \cup \partial^{e} F\right) \cap B\right) \\
& =\left(\theta_{E \cup F} \mathcal{H}\right)\left(\partial^{e} E \cap B\right)+\left(\theta_{E \cup F} \mathcal{H}\right)\left(\partial^{e} F \cap B\right)-\left(\theta_{E \cup F} \mathcal{H}\right)\left(\partial^{e} E \cap \partial^{e} F \cap B\right) \\
& \leq\left(\theta_{E} \mathcal{H}\right)\left(\partial^{e} E \cap B\right)+\left(\theta_{F} \mathcal{H}\right)\left(\partial^{e} F \cap B\right)-\left(\theta_{E \cup F} \mathcal{H}\right)\left(\partial^{e} E \cap \partial^{e} F \cap B\right) \\
& \stackrel{(1.14)}{=} \mathrm{P}(E, B)+\mathrm{P}(F, B)-\left(\theta_{E \cup F} \mathcal{H}\right)\left(\partial^{e} E \cap \partial^{e} F \cap B\right) \\
& =\mathrm{P}(E \cup F, B)-\left(\theta_{E \cup F} \mathcal{H}\right)\left(\partial^{e} E \cap \partial^{e} F \cap B\right),
\end{aligned}
$$

which forces the equality $\left(\theta_{E \cup F} \mathcal{H}\right)\left(\partial^{e} E \cap \partial^{e} F \cap B\right)=0$. Since $\theta_{E \cup F} \geq \gamma_{E \cup F}^{\prime}>0$ on $\partial^{e}(E \cup F)$, we obtain that $\mathcal{H}\left(\partial^{e} E \cap \partial^{e} F \cap \partial^{e}(E \cup F) \cap B\right)=0$. Moreover, we have that

$$
\begin{aligned}
\mathrm{P}(E, B) & =\left(\theta_{E} \mathcal{H}\right)\left(\partial^{e} E \cap \partial^{e}(E \cup F) \cap B\right)+\left(\theta_{E} \mathcal{H}\right)\left(\left(\partial^{e} E \cap B\right) \backslash \partial^{e}(E \cup F)\right) \\
& =\left(\theta_{E \cup F} \mathcal{H}\right)\left(\partial^{e} E \cap \partial^{e}(E \cup F) \cap B\right)+\left(\theta_{E} \mathcal{H}\right)\left(\left(\partial^{e} E \cap B\right) \backslash \partial^{e}(E \cup F)\right) \\
& =\mathrm{P}\left(E \cup F, \partial^{e} E \cap B\right)+\left(\theta_{E} \mathcal{H}\right)\left(\left(\partial^{e} E \cap B\right) \backslash \partial^{e}(E \cup F)\right)
\end{aligned}
$$


and, similarly, that $\mathrm{P}(F, B)=\mathrm{P}\left(E \cup F, \partial^{e} F \cap B\right)+\left(\theta_{F} \mathcal{H}\right)\left(\left(\partial^{e} F \cap B\right) \backslash \partial^{e}(E \cup F)\right)$. This yields

$$
\begin{aligned}
& \mathrm{P}(E \cup F, B)=\mathrm{P}\left(E \cup F, \partial^{e}(E \cup F) \cap B\right) \leq \mathrm{P}\left(E \cup F, \partial^{e} E \cap B\right)+\mathrm{P}\left(E \cup F, \partial^{e} F \cap B\right) \\
& \quad=\mathrm{P}(E, B)+\mathrm{P}(F, B)-\left(\theta_{E} \mathcal{H}\right)\left(\left(\partial^{e} E \cap B\right) \backslash \partial^{e}(E \cup F)\right)-\left(\theta_{F} \mathcal{H}\right)\left(\left(\partial^{e} F \cap B\right) \backslash \partial^{e}(E \cup F)\right) \\
& \quad=\mathrm{P}(E \cup F, B)-\left(\theta_{E} \mathcal{H}\right)\left(\left(\partial^{e} E \cap B\right) \backslash \partial^{e}(E \cup F)\right)-\left(\theta_{F} \mathcal{H}\right)\left(\left(\partial^{e} F \cap B\right) \backslash \partial^{e}(E \cup F)\right) .
\end{aligned}
$$

Hence, we conclude that $\left(\theta_{E} \mathcal{H}\right)\left(\left(\partial^{e} E \cap B\right) \backslash \partial^{e}(E \cup F)\right)=0$ and $\left(\theta_{F} \mathcal{H}\right)\left(\left(\partial^{e} F \cap B\right) \backslash \partial^{e}(E \cup\right.$ $F))=0$. Since $\theta_{E} \geq \gamma_{E}^{\prime}>0$ on $\partial^{e} E$ and $\theta_{F} \geq \gamma_{F}^{\prime}>0$ on $\partial^{e} F$, we get that $\mathcal{H}\left(\left(\partial^{e} E \cap\right.\right.$ $\left.B) \backslash \partial^{e}(E \cup F)\right)=0$ and $\mathcal{H}\left(\left(\partial^{e} F \cap B\right) \backslash \partial^{e}(E \cup F)\right)=0$. In particular, we have $\mathcal{H}\left(\left(\partial^{e} E \cap\right.\right.$ $\left.\left.\partial^{e} F \cap B\right) \backslash \partial^{e}(E \cup F)\right)=0$. Consequently, we have finally proven that $\mathcal{H}\left(\partial^{e} E \cap \partial^{e} F \cap B\right)=0$, as required.

(ii) Let us suppose that $\mathfrak{m}(E \cap F)=0$ and $\mathcal{H}\left(\partial^{e} E \cap \partial^{e} F \cap B\right)=0$. We already know that the inequality $\mathrm{P}(E \cup F, B) \leq \mathrm{P}(E, B)+\mathrm{P}(F, B)$ is always verified. The converse inequality readily follows from our assumptions, item (iv) of Proposition 1.16 and the representation formula for the perimeter measure:

$$
\begin{aligned}
\mathrm{P}(E, B)+\mathrm{P}(F, B)= & \left(\theta_{E} \mathcal{H}\right)\left(\partial^{e} E \cap B\right)+\left(\theta_{F} \mathcal{H}\right)\left(\partial^{e} F \cap B\right) \\
= & \left(\theta_{E} \mathcal{H}\right)\left(\left(\partial^{e} E \backslash \partial^{e} F\right) \cap B\right)+\left(\theta_{E} \mathcal{H}\right)\left(\partial^{e} E \cap \partial^{e} F \cap B\right) \\
& +\left(\theta_{F} \mathcal{H}\right)\left(\left(\partial^{e} F \backslash \partial^{e} E\right) \cap B\right)+\left(\theta_{F} \mathcal{H}\right)\left(\partial^{e} F \cap \partial^{e} E \cap B\right) \\
= & \left(\theta_{E \cup F} \mathcal{H}\right)\left(\left(\partial^{e} E \backslash \partial^{e} F\right) \cap B\right)+\left(\theta_{E \cup F} \mathcal{H}\right)\left(\left(\partial^{e} F \backslash \partial^{e} E\right) \cap B\right) \\
= & \left(\theta_{E \cup F} \mathcal{H}\right)\left(\left(\partial^{e} E \Delta \partial^{e} F\right) \cap B\right) \leq\left(\theta_{E \cup F} \mathcal{H}\right)\left(\partial^{e}(E \cup F) \cap B\right) \\
= & \mathrm{P}(E \cup F, B) .
\end{aligned}
$$

Therefore, it holds that $\mathrm{P}(E \cup F, B)=\mathrm{P}(E, B)+\mathrm{P}(F, B)$, as required.

Remark 2.4 Item (i) of Lemma 2.3 fails for the non-isotropic space in Example 1.27: it holds that $\mathrm{P}\left(R_{1} \cup R_{2}\right)=\mathrm{P}\left(R_{1}\right)+\mathrm{P}\left(R_{2}\right)$, but $\partial^{e} R_{1} \cap \partial^{e} R_{2}=\{o\}$ with $\mathcal{H}(\{o\})>0$.

In the setting of PI spaces having the two-sidedness property, the fact of being an indecomposable set of finite perimeter can be equivalently characterised as illustrated by the following result, which constitutes a generalisation of [21, Proposition 2.12].

Theorem 2.5 Let $(\mathrm{X}, d, \mathfrak{m})$ be a PI space. Then the following properties hold:

(i) Let $E \subset \mathrm{X}$ be a set of finite perimeter such that

$$
f \in L_{\text {loc }}^{1}(\mathfrak{m}),|D f|(\mathrm{X})<+\infty,|D f|\left(E^{1}\right)=0 \Longrightarrow \quad \begin{aligned}
& f=t \text { holds } \mathfrak{m} \text {-a.e. on } E, \\
& \text { for some constant } t \in \mathbb{R} .
\end{aligned}
$$

Then $E$ is indecomposable.

(ii) Suppose $(\mathrm{X}, d, \mathfrak{m})$ has the two-sidedness property. Then any indecomposable subset of $\mathrm{X}$ satisfies (2.1).

Proof (i) Suppose $E \subset \mathrm{X}$ is decomposable. Choose two disjoint sets of finite perimeter $F, G \subset \mathrm{X}$ having positive $\mathrm{m}$-measure such that $E=F \cup G$ and $\mathrm{P}(E)=\mathrm{P}(F)+\mathrm{P}(G)$. Then let us consider the function $f:=\mathbb{1}_{F} \in L_{\text {loc }}^{1}(\mathfrak{m})$. Notice that $|D f|(\mathrm{X})=\mathrm{P}(F)<$ $+\infty$. Moreover, we know from Lemma 1.24 that $\mathcal{H}\left(\partial^{e} F \backslash \partial^{e} E\right)=0$, thus accordingly

$$
|D f|\left(E^{1}\right)=\left(\theta_{F} \mathcal{H}\right)\left(\partial^{e} F \cap E^{1}\right) \leq\left(\theta_{F} \mathcal{H}\right)\left(\partial^{e} E \cap E^{1}\right)=0 .
$$


Nevertheless, $f$ is not $m$-a.e. equal to a constant on $E$, whence $E$ does not satisfy property (2.1).

(ii) Fix an indecomposable set $E \subset \mathrm{X}$. Consider any function $f \in L_{\text {loc }}^{1}(\mathfrak{m})$ such that $|D f|(\mathrm{X})<+\infty$ and $|D f|\left(E^{1}\right)=0$. First of all, we claim that

$$
\mathrm{P}\left(E \cap A, E^{1}\right) \leq \mathrm{P}\left(A, E^{1}\right) \quad \text { for every set } A \subset \mathrm{X} \text { of finite perimeter. }
$$

Indeed, by exploiting the inclusion $\partial^{e}(E \cap A) \subset \partial^{e} E \cup \partial^{e} A$ and the isotropicity of $(\mathrm{X}, d, \mathfrak{m})$ we get

$$
\begin{aligned}
\mathrm{P}\left(E \cap A, E^{1}\right) & =\left(\theta_{E \cap A} \mathcal{H}\right)\left(\partial^{e}(E \cap A) \cap E^{1}\right)=\left(\theta_{E \cap A} \mathcal{H}\right)\left(\partial^{e}(E \cap A) \cap\left(\partial^{e} E \cup \partial^{e} A\right) \cap E^{1}\right) \\
& \leq\left(\theta_{E \cap A} \mathcal{H}\right)\left(\partial^{e}(E \cap A) \cap\left(\partial^{e} E \cap E^{1}\right)\right)+\left(\theta_{E \cap A} \mathcal{H}\right)\left(\partial^{e}(E \cap A) \cap \partial^{e} A \cap E^{1}\right) \\
& =\left(\theta_{E \cap A} \mathcal{H}\right)\left(\partial^{e}(E \cap A) \cap \partial^{e} A \cap E^{1}\right)=\left(\theta_{A} \mathcal{H}\right)\left(\partial^{e}(E \cap A) \cap \partial^{e} A \cap E^{1}\right) \\
& \leq\left(\theta_{A} \mathcal{H}\right)\left(\partial^{e} A \cap E^{1}\right)=\mathrm{P}\left(A, E^{1}\right),
\end{aligned}
$$

whence the claim (2.2) follows. Now let us define the finite Borel measure $\mu$ on $\mathrm{X}$ as

$$
\mu(B):=\int_{\mathbb{R}} \mathrm{P}(\{f>t\}, B) \mathrm{d} t \quad \text { for every Borel set } B \subset \mathrm{X} .
$$

Since $|D f|(\Omega)=\mu(\Omega)$ for every open set $\Omega \subset \mathrm{X}$ by Theorem 1.19 , we deduce that $|D f|=\mu$ by outer regularity. In particular, it holds that $\int_{\mathbb{R}} \mathrm{P}\left(\{f>t\}, E^{1}\right) \mathrm{d} t=$ $|D f|\left(E^{1}\right)=0$, which in turn forces the identity $\mathrm{P}\left(\{f>t\}, E^{1}\right)=0$ for a.e. $t \in \mathbb{R}$. Calling $E_{t}^{+}:=E \cap\{f>t\}$ for all $t \in \mathbb{R}$, we thus infer from (2.2) that $\mathrm{P}\left(E_{t}^{+}, E^{1}\right)=0$ for a.e. $t \in \mathbb{R}$, so that in particular $\mathcal{H}\left(\partial^{e} E_{t}^{+} \cap E^{1}\right)=0$ for a.e. $t \in \mathbb{R}$. Also, we have $\mathcal{H}\left(\partial^{e} E_{t}^{+} \cap \partial^{e}\left(E \backslash E_{t}^{+}\right) \cap \partial^{e} E\right)=0$ for a.e. $t \in \mathbb{R}$ by (1.18), whence

$$
\begin{aligned}
& \mathcal{H}\left(\partial^{e} E_{t}^{+} \cap \partial^{e}\left(E \backslash E_{t}^{+}\right)\right) \\
& \quad \stackrel{(1.6)}{\leq} \mathcal{H}\left(\partial^{e} E_{t}^{+} \cap \partial^{e}\left(E \backslash E_{t}^{+}\right) \cap \partial^{e} E\right)+\mathcal{H}\left(\partial^{e} E_{t}^{+} \cap \partial^{e}\left(E \backslash E_{t}^{+}\right) \cap E^{1}\right) \\
& \quad=\mathcal{H}\left(\partial^{e} E_{t}^{+} \cap \partial^{e}\left(E \backslash E_{t}^{+}\right) \cap E^{1}\right) \leq \mathcal{H}\left(\partial^{e} E_{t}^{+} \cap E^{1}\right)=0
\end{aligned}
$$

holds for a.e. $t \in \mathbb{R}$. Therefore, item (ii) of Lemma 2.3 grants that $\mathrm{P}(E)=\mathrm{P}\left(E_{t}^{+}\right)+$ $\mathrm{P}\left(E \backslash E_{t}^{+}\right)$for a.e. $t \in \mathbb{R}$. Being $E$ indecomposable, we deduce that for a.e. $t \in \mathbb{R}$ we have that either $\mathfrak{m}\left(E_{t}^{+}\right)=0$ or $\mathfrak{m}\left(E \backslash E_{t}^{+}\right)=0$. Define $E_{t}^{-}:=E \cap\{f<t\}$ for all $t \in \mathbb{R}$. Pick a negligible set $N \subset \mathbb{R}$ such that

$$
\text { either } \mathfrak{m}\left(E_{t}^{+}\right)=0 \text { or } \mathfrak{m}\left(E_{t}^{-}\right)=0 \quad \text { for any } t \in \mathbb{R} \backslash N .
$$

Let us define $t_{-}, t_{+} \in \mathbb{R}$ as follows:

$$
\begin{aligned}
& t_{-}:=\sup \left\{t \in \mathbb{R} \backslash N \mid \mathfrak{m}\left(E_{t}^{-}\right)=0\right\}, \\
& t_{+}:=\inf \left\{t \in \mathbb{R} \backslash N \mid \mathfrak{m}\left(E_{t}^{+}\right)=0\right\} .
\end{aligned}
$$

We claim that $\mathfrak{m}\left(E_{t_{-}}^{-}\right)=\mathfrak{m}\left(E_{t_{+}}^{+}\right)=0$. Indeed, given any sequence $\left(t_{n}\right)_{n} \subset \mathbb{R} \backslash N$ such that $t_{n} \nearrow t_{-}$and $\mathfrak{m}\left(E_{t_{n}}^{-}\right)=0$ for all $n \in \mathbb{N}$, we have that $E_{t_{-}}^{-}=\bigcup_{n} E_{t_{n}}^{-}$and accordingly $\mathfrak{m}\left(E_{t_{-}}^{-}\right)=0$. Similarly for $E_{t_{+}}^{+}$. In light of this observation, we see that $t_{-} \leq t_{+}$, otherwise we would have $E=E_{t_{-}}^{-} \cup E_{t_{+}}^{+}$and thus $\mathfrak{m}(E) \leq \mathfrak{m}\left(E_{t_{-}}^{-}\right)+\mathfrak{m}\left(E_{t_{+}}^{+}\right)=0$. We now argue by contradiction: suppose $t_{-}<t_{+}$. Then it holds that $\mathfrak{m}\left(E_{t}^{-}\right), \mathfrak{m}\left(E_{t}^{+}\right)>0$ for every $t \in\left(t_{-}, t_{+}\right) \backslash N$ by definition of $t_{ \pm}$. This leads to a contradiction with (2.3). Then one has $t_{-}=t_{+}$, so that $\mathfrak{m}\left(E \cap\left\{f \neq t_{-}\right\}\right)=\mathfrak{m}\left(E_{t_{-}}^{-}\right)+\mathfrak{m}\left(E_{t_{+}}^{+}\right)=0$. This means that $f=t_{-}$holds $\mathfrak{m}$-a.e. on $E$, which finally shows that $E$ satisfies property (2.1). 
Remark 2.6 In item (ii) of Theorem 2.5, the additional assumptions on (X, $d, \mathfrak{m})$ cannot be dropped. For instance, let us consider the space described in Example 1.29. Calling $E$ the indecomposable set $R_{1} \cup R_{2}$, it holds $E^{1}=E \backslash\{o\}$, thus $\mathbb{1}_{R_{1}} \in \mathrm{BV}(\mathrm{X})$ satisfies $\left|D \mathbb{1}_{R_{1}}\right|\left(E^{1}\right)=$ 0 , but it is not $\mathfrak{m}$-a.e. constant on $E$. This shows that $E$ does not satisfy (2.1).

Corollary 2.7 Let $(\mathrm{X}, d, \mathfrak{m})$ be a PI space. Let $\Omega \subset \mathrm{X}$ be an open, connected set of finite perimeter. Then $\Omega$ is indecomposable.

Proof Let $f \in L_{\text {loc }}^{1}(\mathfrak{m})$ satisfy $|D f|(\mathrm{X})<+\infty$ and $|D f|\left(\Omega^{1}\right)=0$. Being $\Omega$ open, it holds $\Omega^{1} \supset \Omega$, whence $|D f|(\Omega)=0$. Given any $x \in \Omega$, we can choose a radius $r>0$ such that $B_{\lambda r}(x) \subset \Omega$ and accordingly $|D f|\left(B_{\lambda r}(x)\right)=0$, where $\lambda \geq 1$ is the constant appearing in the weak $(1,1)$-Poincaré inequality. Consequently, Lemma 1.13 tells us that $\int_{B_{r}(x)}\left|f-f_{x, r}\right| \mathrm{d} \mathfrak{m}=0$, thus in particular $f$ is $\mathfrak{m}$-a.e. constant on $B_{r}(x)$. This shows that $f$ is locally $\mathfrak{m}$-a.e. constant on $\Omega$. Since $\Omega$ is connected, we deduce that $f$ is $\mathfrak{m}$-a.e. constant on $\Omega$. Therefore, we finally conclude that $\Omega$ is indecomposable by using item (i) of Theorem 2.5 .

Lemma 2.8 Let $(\mathrm{X}, d, \mathfrak{m})$ be an isotropic PI space. Fix a set $E \subset \mathrm{X}$ of finite perimeter and a Borel set $B \subset \mathrm{X}$. Suppose that $\{F, G\}$ is a Borel partition of $E$ such that $P(E, B)=$ $P(F, B)+P(G, B)$. Then it holds that $P(A, B)=P(A \cap F, B)+P(A \cap G, B)$ for every set $A \subset E$ of finite perimeter.

Proof First of all, note that $\mathcal{H}\left(\left(\partial^{e} F \cup \partial^{e} G\right) \cap\left(\partial^{e} E\right)^{c} \cap B\right) \leq \mathcal{H}\left(\partial^{e} F \cap \partial^{e} G \cap B\right)=0$ by item (iv) of Proposition 1.16 and item (i) of Lemma 2.3. This forces the identity

$$
\mathcal{H}\left(\left(\partial^{e} E \Delta\left(\partial^{e} F \cup \partial^{e} G\right)\right) \cap B\right)=0 .
$$

Now fix any set $A \subset E$ of finite perimeter. By using again the property (1.7) we see that

$$
\partial^{e} A \cap B \subset\left(\partial^{e}(A \cap F) \cup \partial^{e}(A \cap G)\right) \cap B .
$$

On the other hand, we claim that

$$
\left(\partial^{e} A\right)^{c} \cap B \subset\left(\left(\partial^{e}(A \cap F) \cup \partial^{e}(A \cap G)\right)^{c} \cup\left(\partial^{e} E\right)^{c}\right) \cap B .
$$

Indeed, pick any $x \in\left(\partial^{e} A\right)^{c}$, thus either $D(A, x)=0$ or $D\left(A^{c}, x\right)=0$. In the former case we deduce that $D(A \cap F, x), D(A \cap G, x) \leq D(A, x)=0$, so that $x \notin \partial^{e}(A \cap F) \cup \partial^{e}(A \cap G)$. In the latter case we have $D\left(E^{c}, x\right) \leq D\left(A^{c}, x\right)=0$, whence $x \notin \partial^{e} E$. This shows the validity of (2.6).

Moreover, notice that $\left(\partial^{e} A\right)^{c} \cap\left(\partial^{e} F\right)^{c} \subset\left(\partial^{e}(A \cap F)\right)^{c}$ and $\left(\partial^{e} A\right)^{c} \cap\left(\partial^{e} G\right)^{c} \subset\left(\partial^{e}(A \cap\right.$ $G))^{c}$ hold by property (1.7), thus accordingly we have that

$$
\left(\partial^{e} A\right)^{c} \cap\left(\partial^{e} F \cup \partial^{e} G\right)^{c} \cap B \subset\left(\partial^{e}(A \cap F) \cup \partial^{e}(A \cap G)\right)^{c} \cap B .
$$

By combining (2.4), (2.5), (2.6) and (2.7), we deduce that

$$
\mathcal{H}\left(\left(\partial^{e} A \Delta\left(\partial^{e}(A \cap F) \cup \partial^{e}(A \cap G)\right)\right) \cap B\right)=0 .
$$

Since $\mathrm{P}(E, B)=\mathrm{P}(F, B)+\mathrm{P}(G, B)$, we know from item (i) of Lemma 2.3 that $\mathcal{H}\left(\partial^{e} F \cap\right.$ $\left.\partial^{e} G \cap B\right)=0$. Property (1.7) ensures that $\partial^{e}(A \cap F) \cap \partial^{e}(A \cap G) \subset \partial^{e} A \cap\left(\partial^{e} F \cap \partial^{e} G\right)$, which together with the identities $\mathcal{H}\left(\partial^{e} F \cap \partial^{e} G \cap B\right)=0$ and (2.8) yield $\mathcal{H}\left(\partial^{e}(A \cap F) \cap \partial^{e}(A \cap\right.$ $G) \cap B)=0$. Therefore, item (ii) of Lemma 2.3 gives $\mathrm{P}(A, B)=\mathrm{P}(A \cap F, B)+\mathrm{P}(A \cap G, B)$, thus proving the statement. 
Corollary 2.9 Let $(\mathrm{X}, d, \mathfrak{m})$ be an isotropic PI space. Fix $E \subset \mathrm{X}$ of finite perimeter and $\Omega \subset$ $\mathrm{X}$ open. Suppose that $\left(E_{n}\right)_{n}$ is a Borel partition of $E$ such that $P(E, \Omega)=\sum_{n=0}^{\infty} P\left(E_{n}, \Omega\right)$. Then

$$
P(F, \Omega)=\sum_{n=0}^{\infty} P\left(F \cap E_{n}, \Omega\right) \quad \text { for every Borel set } F \subset E \text { with } P(F)<+\infty .
$$

Proof Fix any $N \in \mathbb{N}$. By repeatedly applying Lemma 2.8 we obtain that

$$
\mathrm{P}(F, \Omega)=\sum_{n=0}^{N} \mathrm{P}\left(F \cap E_{n}, \Omega\right)+\mathrm{P}\left(\bigcup_{n>N} F \cap E_{n}, \Omega\right) \geq \sum_{n=0}^{N} \mathrm{P}\left(F \cap E_{n}, \Omega\right) .
$$

By letting $N \rightarrow \infty$ we deduce that $\mathrm{P}(F, \Omega) \geq \sum_{n=0}^{\infty} \mathrm{P}\left(F \cap E_{n}, \Omega\right)$, which gives the statement thanks to Remark 2.2.

Proposition 2.10 (Stability of indecomposable sets) Let $(\mathrm{X}, d, \mathfrak{m})$ be an isotropic PI space. Fix a set $E \subset \mathrm{X}$ be offinite perimeter. Let $\left(E_{n}\right)_{n}$ be an increasing sequence of indecomposable subsets of $\mathrm{X}$ such that $E=\bigcup_{n} E_{n}$. Then $E$ is an indecomposable set.

Proof We argue by contradiction: suppose there exists a Borel partition $\{F, G\}$ of the set $E$ such that $\mathfrak{m}(F), \mathfrak{m}(G)>0$ and $\mathrm{P}(E)=\mathrm{P}(F)+\mathrm{P}(G)$. Given that we have $\lim _{n} \mathfrak{m}(F \cap$ $\left.E_{n}\right)=\mathfrak{m}(F)$ and $\lim _{n} \mathfrak{m}\left(G \cap E_{n}\right)=\mathfrak{m}(G)$, we can choose an index $n \in \mathbb{N}$ so that $\mathfrak{m}(F \cap$ $\left.E_{n}\right), \mathfrak{m}\left(G \cap E_{n}\right)>0$. By Lemma 2.8 we know that $\mathrm{P}\left(E_{n}\right)=\mathrm{P}\left(F \cap E_{n}\right)+\mathrm{P}\left(G \cap E_{n}\right)$. Being $\left\{F \cap E_{n}, G \cap E_{n}\right\}$ a Borel partition of $E_{n}$, we get a contradiction with the indecomposability of $E_{n}$. This gives the statement.

Lemma 2.11 Let $(\mathrm{X}, d, \mathfrak{m})$ be an isotropic PI space and $E \subset \mathrm{X}$ a set of finite perimeter. Fix two Borel sets $B, B^{\prime} \subset \mathrm{X}$. Suppose that $E \subset B \subset B^{\prime}$ and that $E$ is indecomposable in $B$. Then it holds that the set $E$ is indecomposable in $B^{\prime}$.

Proof We argue by contradiction: suppose that there exists a Borel partition $\{F, G\}$ of $E$ such that $\mathfrak{m}(F), \mathfrak{m}(G)>0$ and $\mathrm{P}\left(E, B^{\prime}\right)=\mathrm{P}\left(F, B^{\prime}\right)+\mathrm{P}\left(G, B^{\prime}\right)$. Then item (i) of Lemma 2.3 implies that

$$
\mathcal{H}\left(\partial^{e} F \cap \partial^{e} G \cap B\right) \leq \mathcal{H}\left(\partial^{e} F \cap \partial^{e} G \cap B^{\prime}\right)=0,
$$

whence $\mathrm{P}(E, B)=\mathrm{P}(F, B)+\mathrm{P}(G, B)$ by item (ii) of the same lemma. This is in contradiction with the fact that $E$ is indecomposable in $B$, thus the statement is proven.

\subsection{Decomposition theorem}

The aim of this subsection is to show that any set of finite perimeter in an isotropic PI space can be uniquely decomposed into indecomposable sets.

Remark 2.12 Let $\left\{a_{i}^{n}\right\}_{i, n \in \mathbb{N}} \subset(0,+\infty)$ be a sequence that satisfies $\lim _{n} a_{i}^{n}=a_{i}$ for every $i \in \mathbb{N}$ and $\lim _{j} \varlimsup_{\lim _{n}} \sum_{i=j}^{\infty} a_{i}^{n}=0$. Then $\sum_{i=0}^{\infty} a_{i}=\lim _{n} \sum_{i=0}^{\infty} a_{i}^{n}$. Indeed, for every $j \in \mathbb{N}$ we have that

$$
\begin{aligned}
\sum_{i=0}^{j} a_{i} & =\lim _{n \rightarrow \infty} \sum_{i=0}^{j} a_{i}^{n} \leq \lim _{n \rightarrow \infty} \sum_{i=0}^{\infty} a_{i}^{n} \leq \varlimsup_{n \rightarrow \infty} \sum_{i=0}^{\infty} a_{i}^{n}=\varlimsup_{n \rightarrow \infty}\left[\sum_{i=0}^{j} a_{i}^{n}+\sum_{i>j} a_{i}^{n}\right] \\
& =\sum_{i=0}^{j} a_{i}+\varlimsup_{n \rightarrow \infty} \sum_{i>j} a_{i}^{n},
\end{aligned}
$$


whence by letting $j \rightarrow \infty$ we conclude that $\sum_{i=0}^{\infty} a_{i} \leq \underline{\lim }_{n} \sum_{i=0}^{\infty} a_{i}^{n} \leq \varlimsup_{\lim } \sum_{i=0}^{\infty} a_{i}^{n} \leq$ $\sum_{i=0}^{\infty} a_{i}$, which proves the claim.

Proposition 2.13 Let $(\mathrm{X}, d, \mathfrak{m})$ be an isotropic PI space. Let $E \subset \mathrm{X}$ be a set of finite perimeter. Fix $\bar{x} \in \mathrm{X}$ and $r>0$ such that $B_{r}(\bar{x})$ has finite perimeter. Then there is $a$ unique (in the $\mathfrak{m}$-a.e. sense) at most countable partition $\left\{E_{i}\right\}_{i \in I}$ of $E \cap B_{r}(\bar{x})$, into indecomposable subsets of $B_{r}(\bar{x})$, such that $P\left(E_{i}\right)<+\infty, \mathfrak{m}\left(E_{i}\right)>0$ for every $i \in I$ and $P\left(E, B_{r}(\bar{x})\right)=\sum_{i \in I} P\left(E_{i}, B_{r}(\bar{x})\right)$. Moreover, the sets $\left\{E_{i}\right\}_{i \in I}$ are maximal indecomposable sets, meaning that for any Borel set $F \subset E \cap B_{r}(\bar{x})$ with $P(F)<+\infty$ that is indecomposable in $B_{r}(\bar{x})$ there is a (unique) $i \in I$ such that $\mathfrak{m}\left(F \backslash E_{i}\right)=0$.

Proof EXISTENCE. Fix an exponent $s>\max \left\{1, \log _{2}\left(C_{D}\right)\right\}$ and any $\alpha \in\left(1, \frac{s}{s-1}\right)$. For brevity, call $\Omega:=B_{r}(\bar{x})$. For simplicity, let us set

$$
\mu(B):=\mathfrak{m}(B)^{1 / \alpha} \quad \text { for every Borel set } B \subset \Omega .
$$

Let us denote by $\mathcal{P}$ the collection of all Borel partitions $\left(E_{i}\right)_{i \in \mathbb{N}}$ of $E \cap \Omega$ (up to m-null sets) such that $\left(\mathfrak{m}\left(E_{i}\right)\right)_{i \in \mathbb{N}}$ is non-increasing, $\sum_{i=0}^{\infty} \mathrm{P}\left(E_{i}, \Omega\right) \leq \mathrm{P}(E, \Omega)$, and $\sum_{i=0}^{\infty} \mathrm{P}\left(E_{i}\right) \leq$ $\mathrm{P}(E)+\mathrm{P}(\Omega)$. Note that the family $\mathcal{P}$ is non-empty, as it contains the element $(E \cap \Omega, \emptyset, \emptyset, \ldots)$. Let us call

$$
M:=\sup \left\{\sum_{i=0}^{\infty} \mu\left(E_{i}\right) \mid\left\{E_{i}\right\}_{i \in \mathbb{N}} \in \mathcal{P}\right\} .
$$

Choose any $\left(\left(E_{i}^{n}\right)_{i \in \mathbb{N}}\right)_{n} \subset \mathcal{P}$ such that $\lim _{n} \sum_{i=0}^{\infty} \mu\left(E_{i}^{n}\right)=M$. Since $\mathrm{P}\left(E_{i}^{n}\right) \leq \mathrm{P}(E)+$ $\mathrm{P}(\Omega)<+\infty$ for every $i, n \in \mathbb{N}$, we know by the compactness properties of sets of finite perimeter that we can extract a (not relabelled) subsequence in $n$ in such a way that the following property holds: there exists a sequence $\left(E_{i}\right)_{i \in \mathbb{N}}$ of Borel subsets of $E \cap \Omega$ such that $\mathbb{1}_{E_{i}^{n}} \rightarrow \mathbb{1}_{E_{i}}$ in $L^{1}(\mathfrak{m}\llcorner\Omega)$, thus

$$
\lim _{n \rightarrow \infty} \mu\left(E_{i}^{n}\right)=\mu\left(E_{i}\right) \quad \text { for every } i \in \mathbb{N} .
$$

Given any $i, j \in \mathbb{N}$ such that $i \neq j$, we also have that $\mu\left(E_{i} \cap E_{j}\right)=\lim _{n} \mu\left(E_{i}^{n} \cap E_{j}^{n}\right)=0$, thus accordingly $\mathfrak{m}\left(E_{i} \cap E_{j}\right)=0$. Moreover, by lower semicontinuity of the perimeter we see that

$$
\begin{aligned}
\sum_{i=0}^{\infty} \mathrm{P}\left(E_{i}, \Omega\right) & =\lim _{j \rightarrow \infty} \sum_{i=0}^{j} \mathrm{P}\left(E_{i}, \Omega\right) \leq \lim _{j \rightarrow \infty} \sum_{i=0}^{j} \underset{n \rightarrow \infty}{\lim } \mathrm{P}\left(E_{i}^{n}, \Omega\right) \\
& \leq \lim _{j \rightarrow \infty} \underset{n \rightarrow \infty}{\lim } \sum_{i=0}^{j} \mathrm{P}\left(E_{i}^{n}, \Omega\right) \leq \mathrm{P}(E, \Omega)
\end{aligned}
$$

and, similarly, that $\sum_{i=0}^{\infty} \mathrm{P}\left(E_{i}\right) \leq \mathrm{P}(E)+\mathrm{P}(\Omega)$ for every $i \in \mathbb{N}$. To prove that $\left(E_{i}\right)_{i \in \mathbb{N}} \in \mathcal{P}$ it only remains to show that $\mathfrak{m}\left((E \cap \Omega) \backslash \bigcup_{i} E_{i}\right)=0$. We claim that

$$
\lim _{j \rightarrow \infty} \varlimsup_{n \rightarrow \infty} \sum_{i=j}^{\infty} \mu\left(E_{i}^{n}\right)^{\alpha} \leq \lim _{j \rightarrow \infty} \varlimsup_{n \rightarrow \infty} \sum_{i=j}^{\infty} \mu\left(E_{i}^{n}\right)=0 .
$$

Observe that the inequality $\mathfrak{m}\left(E_{i}^{n}\right) \leq \mathfrak{m}\left(\Omega \backslash E_{i}^{n}\right)$ holds for every $i \geq 1$. Let us define

$$
\eta:=\frac{1}{\alpha}-\frac{s-1}{s}>0, \quad C:=C_{I}\left(\frac{r^{s}}{\mathfrak{m}(\Omega)}\right)^{1 / s-1} .
$$


We readily deduce from the relative isoperimetric inequality (1.8) that for all $j \geq 1$ we have

$$
\begin{aligned}
j \mathfrak{m}\left(E_{j}^{n}\right) & \leq \sum_{i=1}^{j} \mathfrak{m}\left(E_{i}^{n}\right) \leq C \sum_{i=1}^{j} \mathrm{P}\left(E_{i}^{n}, B_{2 \lambda r}(\bar{x})\right)^{s / s-1}=C \sum_{i=1}^{j} \mathrm{P}\left(E_{i}^{n}\right)^{s / s-1} \\
& =C[\mathrm{P}(E)+\mathrm{P}(\Omega)]^{s / s-1} \sum_{i=1}^{j}\left(\frac{\mathrm{P}\left(E_{i}^{n}\right)}{\mathrm{P}(E)+\mathrm{P}(\Omega)}\right)^{s / s-1} \\
& \leq C[\mathrm{P}(E)+\mathrm{P}(\Omega)]^{s / s-1} \sum_{i=1}^{j} \frac{\mathrm{P}\left(E_{i}^{n}\right)}{\mathrm{P}(E)+\mathrm{P}(\Omega)} \\
& \leq C[\mathrm{P}(E)+\mathrm{P}(\Omega)]^{s / s-1} .
\end{aligned}
$$

Furthermore, by using the previous estimate and again (1.14) we obtain that

$$
\begin{aligned}
\sum_{i=j}^{\infty} \mu\left(E_{i}^{n}\right) & =\sum_{i=j}^{\infty} \mathfrak{m}\left(E_{i}^{n}\right)^{1 / \alpha}=\sum_{i=j}^{\infty} \mathfrak{m}\left(E_{i}^{n}\right)^{\eta} \mathfrak{m}\left(E_{i}^{n}\right)^{(s-1) / s} \leq \mathfrak{m}\left(E_{j}^{n}\right)^{\eta} \sum_{i=j}^{\infty} \mathfrak{m}\left(E_{i}^{n}\right)^{(s-1) / s} \\
& \leq \frac{C^{\eta}[\mathrm{P}(E)+\mathrm{P}(\Omega)]^{\eta s / s-1}}{j^{\eta}} \sum_{i=j}^{\infty} \mathfrak{m}\left(E_{i}^{n}\right)^{(s-1) / s} \\
& \leq \frac{C^{\eta}[\mathrm{P}(E)+\mathrm{P}(\Omega)]^{\eta s / s-1}}{j^{\eta}} C^{(s-1) / s} \sum_{i=j}^{\infty} \mathrm{P}\left(E_{i}^{n}, B_{2 \lambda r}(\bar{x})\right) \\
& =\frac{C^{1 / \alpha}[\mathrm{P}(E)+\mathrm{P}(\Omega)]^{\eta s / s-1}}{j^{\eta}} \sum_{i=j}^{\infty} \mathrm{P}\left(E_{i}^{n}\right) \\
& =\frac{C^{1 / \alpha}[\mathrm{P}(E)+\mathrm{P}(\Omega)]^{\eta s / s-1+1}}{j^{\eta}}=\frac{C^{1 / \alpha}[\mathrm{P}(E)+\mathrm{P}(\Omega)]^{s / \alpha(s-1)}}{j^{\eta}} .
\end{aligned}
$$

Consequently, we deduce that the claim (2.11) is verified. By recalling also (2.10) and Remark 2.12, we can conclude that

$$
\begin{aligned}
\mu\left(\bigcup_{i \in \mathbb{N}} E_{i}\right)^{\alpha} & =\mathfrak{m}\left(\bigcup_{i \in \mathbb{N}} E_{i}\right)=\sum_{i=0}^{\infty} \mathfrak{m}\left(E_{i}\right)=\sum_{i=0}^{\infty} \mu\left(E_{i}\right)^{\alpha} \\
& =\lim _{n \rightarrow \infty} \sum_{i=0}^{\infty} \mu\left(E_{i}^{n}\right)^{\alpha}=\mu(E \cap \Omega)^{\alpha}
\end{aligned}
$$

This forces $\mathfrak{m}\left((E \cap \Omega) \backslash \bigcup_{i} E_{i}\right)=0$ and accordingly $\left(E_{i}\right)_{i \in \mathbb{N}} \in \mathcal{P}$. Hence,

$$
\sum_{i=0}^{\infty} \mu\left(E_{i}\right)=\lim _{n \rightarrow \infty} \sum_{i=0}^{\infty} \mu\left(E_{i}^{n}\right)=M,
$$

in other words $\left(E_{i}\right)_{i \in \mathbb{N}}$ is a maximiser for the problem in (2.9). Finally, we claim that each set $E_{i}$ is indecomposable in $\Omega$. Suppose this was not the case: then for some $j \in \mathbb{N}$ we would find a partition $\{F, G\}$ of $E_{j}$ into sets of finite perimeter having positive $\mathfrak{m}$-measure and satisfying the identity $\mathrm{P}\left(E_{j}, \Omega\right)=\mathrm{P}(F, \Omega)+\mathrm{P}(G, \Omega)$. We can relabel the family $\left\{E_{i}\right\}_{i \neq j} \cup\{F, G\}$ as $\left(F_{i}\right)_{i \in \mathbb{N}}$ in such a way that $\left(\mathfrak{m}\left(F_{i}\right)\right)_{i \in \mathbb{N}}$ is a non-increasing sequence. Given that 


$$
\sum_{i=0}^{\infty} \mathrm{P}\left(F_{i}, \Omega\right)=\sum_{i \neq j} \mathrm{P}\left(E_{i}, \Omega\right)+\mathrm{P}(F, \Omega)+\mathrm{P}(G, \Omega)=\sum_{i=0}^{\infty} \mathrm{P}\left(E_{i}, \Omega\right) \leq \mathrm{P}(E, \Omega),
$$

we see that $\left(F_{i}\right)_{i \in \mathbb{N}} \in \mathcal{P}$. On the other hand, given that $\alpha>1$ and $\mu(F), \mu(G)>0$ we have the inequality $\mu(F)+\mu(G)>\mu\left(E_{j}\right)$, so that

$$
\sum_{i=0}^{\infty} \mu\left(F_{i}\right)=\sum_{i \neq j} \mu\left(E_{i}\right)+\mu(F)+\mu(G)>\sum_{i=0}^{\infty} \mu\left(E_{i}\right)=M .
$$

This leads to a contradiction with (2.9), whence the sets $E_{i}$ are proven to be indecomposable in $\Omega$. Therefore, the family $\left\{E_{i}\right\}_{i \in I}$, where $I:=\left\{i \in \mathbb{N}: \mathfrak{m}\left(E_{i}\right)>0\right\}$, satisfies the required properties.

MAXIMALITy. Let $F \subset E \cap \Omega$ be a fixed Borel set with $\mathrm{P}(F)<+\infty$ that is indecomposable in $\Omega$. Choose an index $j \in I$ for which $\mathfrak{m}\left(F \cap E_{j}\right)>0$. By Corollary 2.9 we know that

$$
\mathrm{P}\left(F \cap E_{j}, \Omega\right)+\mathrm{P}\left(F \cap \bigcup_{i \neq j} E_{i}, \Omega\right)=\mathrm{P}\left(F \cap E_{j}, \Omega\right)+\sum_{i \neq j} \mathrm{P}\left(F \cap E_{i}, \Omega\right)=\mathrm{P}(F, \Omega) .
$$

Given that $F$ is assumed to be indecomposable in $\Omega$, we finally conclude that $F \cap \bigcup_{i \neq j} E_{i}$ has null $\mathfrak{m}$-measure, so that $\mathfrak{m}\left(F \backslash E_{j}\right)=0$. This shows that the elements of $\left\{E_{i}\right\}_{i \in I}$ are maximal.

UNIQUENESS. Consider any other family $\left\{F_{j}\right\}_{j \in J}$ having the same properties as $\left\{E_{i}\right\}_{i \in I}$. By maximality we know that for any $i \in \mathbb{N}$ there exists a (unique) $j \in \mathbb{N}$ such that $\mathfrak{m}\left(E_{i} \Delta F_{j}\right)=0$, thus the two partitions $\left\{E_{i}\right\}_{i \in I}$ and $\left\{F_{j}\right\}_{j \in J}$ are essentially equivalent (up to $\mathfrak{m}$-negligible sets). This proves the desired uniqueness.

We are now ready to prove the main result of this section:

Theorem 2.14 (Decomposition theorem) Let $(\mathrm{X}, d, \mathfrak{m})$ be an isotropic PI space. Let $E \subset \mathrm{X}$ be a set of finite perimeter. Then there exists a unique (finite or countable) partition $\left\{E_{i}\right\}_{i \in I}$ of $E$ into indecomposable subsets of $\mathrm{X}$ such that $\mathfrak{m}\left(E_{i}\right)>0$ for every $i \in I$ and $P(E)=$ $\sum_{i \in I} P\left(E_{i}\right)$, where uniqueness has to be intended in the $\mathfrak{m}$-a.e. sense. Moreover, the sets $\left\{E_{i}\right\}_{i \in I}$ are maximal indecomposable sets, meaning that for any Borel set $F \subset E$ with $P(F)<+\infty$ that is indecomposable there is a (unique) $i \in I$ such that $\mathfrak{m}\left(F \backslash E_{i}\right)=0$.

Proof Let $\bar{x} \in \mathrm{X}$ be a fixed point. Choose a sequence of radii $r_{j} \nearrow+\infty$ such that $\Omega_{j}:=B_{r_{j}}(\bar{x})$ has finite perimeter for all $j \in \mathbb{N}$. Let us apply Proposition 2.13: given any $j \in \mathbb{N}$, there exists an m-essentially unique partition $\left\{E_{i}^{j}\right\}_{i \in I_{j}}$ of $E \cap \Omega_{j}$, into sets of finite perimeter that are maximal indecomposable subsets of $\Omega_{j}$, with $\mathfrak{m}\left(E_{i}^{j}\right)>0$ for all $i \in I_{j}$ and $\mathrm{P}\left(E, \Omega_{j}\right)=\sum_{i \in I_{j}} \mathrm{P}\left(E_{i}^{j}, \Omega_{j}\right)$.

Given any $j \in \mathbb{N}$ and $i \in I_{j}$, we know from Lemma 2.11 that $E_{i}^{j}$ is indecomposable in $\Omega_{j+1}$, thus there exists $\ell \in I_{j+1}$ for which $\mathfrak{m}\left(E_{i}^{j} \backslash E_{\ell}^{j+1}\right)=0$. This ensures thatpossibly choosing different $\mathfrak{m}$-a.e. representatives of the sets $E_{i}^{j}$,s under consideration-we can assume that:

For every $j \in \mathbb{N}$ and $i \in I_{j}$ there exists (a unique) $\ell \in I_{j+1}$ such that $E_{i}^{j} \subset E_{\ell}^{j+1}$.

Given any $x \in E$, let us define the set $G_{x} \subset E$

$$
G_{x}:=\bigcup\left\{E_{i}^{j} \mid j \in \mathbb{N}, i \in I_{j}, x \in E_{i}^{j}\right\} .
$$


One clearly has that $\mathfrak{m}\left(G_{x}\right)>0$. Moreover, it readily follows from (2.13) that

$$
G_{x}=\bigcup\left\{E_{i}^{j} \mid j \in \mathbb{N}, j \geq j_{0}, i \in I_{j}, x \in E_{i}^{j}\right\} \quad \text { for every } j_{0} \in \mathbb{N} .
$$

We claim that:

$$
\text { For every } x, y \in E \text { it holds that either } G_{x} \cap G_{y}=\emptyset \text { or } G_{x}=G_{y} .
$$

In order to prove it, assume that $G_{x} \cap G_{y} \neq \emptyset$ and pick any $z \in G_{x} \cap G_{y}$. Then there exist some indices $j_{x}, j_{y} \in \mathbb{N}, i_{x} \in I_{j_{x}}$ and $i_{y} \in I_{j_{y}}$ such that $\{x, z\} \subset E_{i_{x}}^{j_{x}}$ and $\{y, z\} \subset E_{i_{y}}^{j_{y}}$. Possibly interchanging $x$ and $y$, we can suppose that $j_{y} \leq j_{x}$. Given that $E_{i_{x}}^{j_{x}} \cap E_{i_{y}}^{j_{y}}$ is not empty (as it contains $z$ ), we infer from (2.13) that $E_{i_{y}}^{j_{y}} \subset E_{i_{x}}^{j_{x}}$. Consequently, property (2.14) ensures that the sets $G_{x}$ and $G_{y}$ coincide, thus proving the claim (2.15).

Let us define $\mathcal{F}:=\left\{G_{x}: x \in E\right\}$. It turns out that the family $\mathcal{F}$ is at most countable: the map sending each element $(j, i)$ of $\bigsqcup_{j \in \mathbb{N}} I_{j}$ to the unique element of $\mathcal{F}$ containing $E_{i}^{j}$ is clearly surjective. Then rename $\mathcal{F}$ as $\left\{E_{i}\right\}_{i \in I}$. Observe that $\left\{E_{i}\right\}_{i \in I}$ constitutes a Borel partition of $E$. Now fix $i \in I$. We can choose $j(i) \in \mathbb{N}$ and $\ell(i, j) \in I_{j}$ for all $j \geq j(i)$ such that $E_{i}=\bigcup_{j \geq j(i)} E_{\ell(i, j)}^{j}$. Let us also call

$$
F_{i}^{j}:= \begin{cases}\emptyset & \text { if } j<j(i), \\ E_{\ell(i, j)}^{j} & \text { if } j \geq j(i) .\end{cases}
$$

Therefore, $E_{i}=\bigcup_{j \in \mathbb{N}} F_{i}^{j}$. Given any $j \in \mathbb{N}$, we have $\mathrm{P}\left(E_{i} \cap \Omega_{j}, \Omega_{j}\right)=\mathrm{P}\left(E_{i}, \Omega_{j}\right)$ as $\Omega_{j}$ is open. Then $\mathrm{P}\left(E_{i}, \Omega_{j}\right)=\mathrm{P}\left(E_{i} \cap \Omega_{j}, \Omega_{j}\right)=\mathrm{P}\left(F_{i}^{j}, \Omega_{j}\right) \leq \mathrm{P}\left(E, \Omega_{j}\right) \leq \mathrm{P}(E)$ holds for every $j \geq j(i)$, so that

$$
\mathrm{P}\left(E_{i}\right)=\lim _{j \rightarrow \infty} \mathrm{P}\left(E_{i}, \Omega_{j}\right) \leq \mathrm{P}(E) .
$$

This shows that the sets $\left\{E_{i}\right\}_{i \in I}$ have finite perimeter, while the fact that they are indecomposable follows from Proposition 2.10. Now fix any finite subset $J$ of $I$. Similarly to the estimates above, we see that for every $j \geq \max \{j(i): i \in J\}$ it holds that

$$
\begin{aligned}
\sum_{i \in J} \mathrm{P}\left(E_{i}, \Omega_{j}\right) & =\sum_{i \in J} \mathrm{P}\left(E_{i} \cap \Omega_{j}, \Omega_{j}\right)=\sum_{i \in J} \mathrm{P}\left(F_{i}^{j}, \Omega_{j}\right) \leq \sum_{\ell \in I_{j}} \mathrm{P}\left(E_{\ell}^{j}, \Omega_{j}\right) \\
& \leq \mathrm{P}\left(E, \Omega_{j}\right) \leq \mathrm{P}(E),
\end{aligned}
$$

whence $\sum_{i \in J} \mathrm{P}\left(E_{i}\right)=\lim _{j} \sum_{i \in J} \mathrm{P}\left(E_{i}, \Omega_{j}\right) \leq \mathrm{P}(E)$. By arbitrariness of $J \subset I$ this yields the inequality $\sum_{i \in I} \mathrm{P}\left(E_{i}\right) \leq \mathrm{P}(E)$, thus accordingly $\mathrm{P}(E)=\sum_{i \in I} \mathrm{P}\left(E_{i}\right)$ by Remark 2.2. Finally, maximality and uniqueness can be proven by arguing exactly as in Proposition 2.13. Therefore, the statement is achieved.

Definition 2.15 (Essential connected components) Let $(\mathrm{X}, d, \mathfrak{m})$ be an isotropic PI space. Let us fix a set $E \subset X$ of finite perimeter. Then we denote by

$$
\mathcal{C C}^{e}(E):=\left\{E_{i}\right\}_{i \in I}
$$

the decomposition of $E$ provided by Theorem 2.14. (We assume the index set is either $I=\mathbb{N}$ or $I=\{0, \ldots, n\}$ for some $n \in \mathbb{N}$.) The sets $E_{i}$ are called the essential connected components of $E$. 
Example 2.16 Although we do not know if the Decomposition Theorem 2.14 holds without the assumption on isotropicity, one can see that the assumption on $(1,1)$-Poincaré inequality cannot be relaxed to a $(1, p)$-Poincaré inequality with $p>1$. As an example of this, one can take a fat Sierpiński carpet $S_{\mathbf{a}} \subset[0,1]^{2}$ with a sequence $\mathbf{a} \in \ell^{2} \backslash \ell^{1}$, as defined in [35]. The set $S_{\mathrm{a}}$, equipped with a natural measure $\mathfrak{m}$ and distance $d$, is a 2-Ahlfors-regular metric measure space supporting a $(1, p)$-Poincaré inequality for all exponents $p>1$. Nevertheless, given any vertical strip of the form $I_{x, \varepsilon}:=(x-\varepsilon, x+\varepsilon) \times[0,1]$, where $x=\sum_{i=1}^{n} x_{i} 3^{-i}+2^{-1} 3^{-n}$ with $x_{i} \in\{0,1,2\}$ and $n \in \mathbb{N}$, we have $\mathfrak{m}\left(I_{x, \varepsilon}\right) / \varepsilon \rightarrow 0$ as $\varepsilon \rightarrow 0$. Thus, any set of finite perimeter $E \subset S_{\mathbf{a}}$ can be decomposed into the union of $E \cap([0, x] \times[0,1])$ and $E \cap([x, 1] \times[0,1])$. Since the family of coordinates $x$ for which this holds is dense in $[0,1]$, no set of positive measure in $S_{\mathrm{a}}$ can be decomposed into countably many indecomposable sets.

Remark 2.17 Given an isotropic PI space and a set $E \subset \mathrm{X}$ of finite perimeter, it holds that

$$
\mathcal{H}\left(\partial^{e} F \backslash \partial^{e} E\right)=0 \quad \text { for every } F \in \mathcal{C C}^{e}(E) .
$$

This property is an immediate consequence of Lemma 1.24.

Proposition 2.18 (Stability of indecomposable sets, II) Let $(\mathrm{X}, d, \mathfrak{m})$ be an isotropic PI space. Fix two indecomposable sets $E, F \subset \mathrm{X}$. Suppose that either $\mathfrak{m}(E \cap F)>0$ or $\mathcal{H}\left(\partial^{e} E \cap\right.$ $\left.\partial^{e} F\right)>0$. Then $E \cup F$ is an indecomposable set.

Proof Denote $\mathcal{C C}^{e}(E \cup F)=\left\{G_{i}\right\}_{i \in I}$. Choose $i, j \in I$ such that $\mathfrak{m}\left(E \backslash G_{i}\right)=\mathfrak{m}\left(F \backslash G_{j}\right)=0$, whose existence is granted by the maximality of the connected components of $E \cup F$. If $\mathfrak{m}(E \cap$ $F)>0$ then $i=j$, whence $\mathcal{C} \mathcal{C}^{e}(E \cup F)=\{E \cup F\}$ and accordingly $E \cup F$ is indecomposable. Otherwise, we have $i \neq j$ and $\mathcal{C C}^{e}(E \cup F)=\{E, F\}$, so that $\mathcal{H}\left(\partial^{e} E \cap \partial^{e} F\right)=0$ by item (i) of Lemma 2.3 .

Remark 2.19 Let us highlight the two main technical differences between the proofs we carried out in this section and the corresponding ones for $\mathbb{R}^{n}$ that were originally presented in [4]:

(i) There exist isotropic PI spaces $\mathrm{X}$ where it is possible to find a set of finite perimeter $E$ whose associated perimeter measure $\mathrm{P}(E, \cdot)$ is not concentrated on $E^{1 / 2}$. For instance, consider the space described in Example 1.29: it is an isotropic PI space where (1.18) fails, thus in particular property (1.19) is not verified (as a consequence of Lemma 1.30). Some of the results of [4] — which have a counterpart in this paper-are proven by using property (1.19). Consequently, the approaches we followed to prove some of the results of this section provide new proofs even in the Euclidean setting.

(ii) An essential ingredient in the proof of the decomposition theorem [4, Theorem 1] is the (global) isoperimetric inequality. In our case, we only have the relative isoperimetric inequality at disposal, thus we need to 'localise' the problem: first we prove a local version of the decomposition theorem (namely, Proposition 2.13), then we obtain the full decomposition by means of a 'patching argument' (as described in the proof of Theorem 2.14). Let us point out that in the Ahlfors-regular case the proof of the decomposition theorem would closely follows along the lines of [4, Theorem 1] (thanks to Theorem 1.18).

Finally, an alternative proof of the decomposition theorem will be provided in Sect. 4. 


\section{Extreme points in the space of BV functions}

The aim of Sect. 3.1 is to study the extreme points of the 'unit ball' in the space of BV functions over an isotropic PI space (with a uniform bound on the support). More precisely, given an isotropic PI space $(\mathrm{X}, d, \mathfrak{m})$ and a compact set $K \subset \mathrm{X}$, we will detect the extreme points of the convex set made of all functions $f \in \mathrm{BV}(\mathrm{X})$ such that $\operatorname{spt}(f) \subset K$ and $|D f|(\mathrm{X}) \leq 1$, with respect to the strong topology of $L^{1}(\mathfrak{m})$; cf. Theorem 3.8. Informally speaking, the extreme points coincide — at least under some further assumptions-with the (suitably normalised) characteristic functions of simple sets, whose definition is given in Definition 3.1. In Sect. 3.2 we provide an alternative characterisation of simple sets (cf. Theorem 3.17) in the framework of Alhfors-regular spaces, a key role being played by the concept of saturation of a set, whose definition relies upon the decomposition properties treated in Sect. 2.

\subsection{Simple sets and extreme points in BV}

A set of finite perimeter $E \subset \mathbb{R}^{n}$ having finite Lebesgue measure is a simple set provided one of the following (equivalent) properties is satisfied:

(i) $E$ is indecomposable and saturated, the latter term meaning that the complement of $E$ does not have essential connected components of finite Lebesgue measure.

(ii) Both $E$ and $\mathbb{R}^{n} \backslash E$ are indecomposable.

(iii) If $F \subset \mathbb{R}^{n}$ is a set of finite perimeter such that $\partial^{e} F$ is essentially contained in $\partial^{e} E$ (with respect to the $(n-1)$-dimensional Hausdorff measure) and $\mathcal{L}^{n}(F)<+\infty$, then it holds that $F=E$ (up to $\mathcal{L}^{n}$-null sets).

We refer to [4, Section 5] for a discussion about the equivalence of the above conditions. In the more general setting of isotropic PI spaces, (the appropriate reformulations of) these three notions are no longer equivalent. The one that well captures the property we are interested in (i.e., the fact of providing an alternative characterisation of the extreme points in BV) is item (iii), which accordingly is the one that we choose as the definition of simple set in our context:

Definition 3.1 (Simple sets) Let $(\mathrm{X}, d, \mathfrak{m})$ be a PI space. Let $E \subset \mathrm{X}$ be a set of finite perimeter with $\mathfrak{m}(E)<+\infty$. Then we say that $E$ is a simple set provided for every set $F \subset \mathrm{X}$ of finite perimeter with $\mathcal{H}\left(\partial^{e} F \backslash \partial^{e} E\right)=0$ it holds $\mathfrak{m}(F)=0, \mathfrak{m}\left(F^{c}\right)=0, \mathfrak{m}(F \Delta E)=0$, or $\mathfrak{m}\left(F \Delta E^{c}\right)=0$.

It is rather easy to prove that - under some additional assumptions - the definition of simple set we have just proposed is equivalent to (the suitable rephrasing of) item (ii) above:

Proposition 3.2 (Indecomposability of simple sets) Let $(\mathrm{X}, d, \mathfrak{m})$ be an isotropic PI space. Let us consider a set $E \subset \mathrm{X}$ of finite perimeter such that $\mathfrak{m}(E)<+\infty$. Then:

(i) If $E$ is a simple set, then $E$ and $E^{c}$ are indecomposable.

(ii) Suppose that $(\mathrm{X}, d, \mathfrak{m})$ has the two-sidedness property. If $E$ and $E^{c}$ are indecomposable, then $E$ is a simple set.

Proof (i) Assume $E \subset \mathrm{X}$ is a simple set. First, we prove by contradiction that $E$ is indecomposable: suppose it is not, thus it can be written as $E=F \cup G$ for some pairwise disjoint sets 
$F, G$ of finite perimeter such that $\mathfrak{m}(F), \mathfrak{m}(G)>0$ and $\mathrm{P}(E)=\mathrm{P}(F)+\mathrm{P}(G)$. By combining item (iv) of Proposition 1.16 with item (ii) of Lemma 2.3, we obtain that

$$
\mathcal{H}\left(\left(\partial^{e} F \cup \partial^{e} G\right) \backslash \partial^{e} E\right) \leq \mathcal{H}\left(\partial^{e} F \cap \partial^{e} G\right)=0 .
$$

In particular, we have that $\mathcal{H}\left(\partial^{e} F \backslash \partial^{e} E\right)=\mathcal{H}\left(\partial^{e} G \backslash \partial^{e} E\right)=0$. Being $E$ simple, we get $F=G=E$, which leads to a contradiction. Then $E$ is indecomposable. In order to show that also $E^{c}$ is indecomposable, we argue in a similar way: suppose $E^{c}=F^{\prime} \cup G^{\prime}$ for pairwise disjoint sets $F^{\prime}, G^{\prime}$ of finite perimeter with $\mathfrak{m}\left(F^{\prime}\right), \mathfrak{m}\left(G^{\prime}\right)>0$ and $\mathrm{P}\left(E^{c}\right)=\mathrm{P}\left(F^{\prime}\right)+\mathrm{P}\left(G^{\prime}\right)$. By arguing as before we obtain that $\mathcal{H}\left(\partial^{e} E^{c} \backslash \partial^{e} F^{\prime}\right)=\mathcal{H}\left(\partial^{e} E^{c} \backslash \partial^{e} F^{\prime}\right)=0$. Being $\partial^{e} E^{c}=\partial^{e} E$, we can conclude (again since $E$ is simple) that $F^{\prime}=G^{\prime}=E^{c}$, whence the contradiction. Therefore, $E^{c}$ is indecomposable.

(ii) Assume that $(\mathrm{X}, d, \mathfrak{m})$ has the two-sidedness property and that $E, E^{c}$ are indecomposable sets. Take a set $F \subset \mathrm{X}$ of finite perimeter such that $\mathcal{H}\left(\partial^{e} F \backslash \partial^{e} E\right)=0$. We know from (1.18) that

$$
\mathcal{H}\left(\partial^{e}(E \cap F) \cap \partial^{e}(E \backslash F) \cap \partial^{e} E\right)=0=\mathcal{H}\left(\partial^{e}\left(E^{c} \cap F\right) \cap \partial^{e}\left(E^{c} \backslash F\right) \cap \partial^{e} E^{c}\right) .
$$

Consequently, we deduce that

$$
\begin{aligned}
\mathcal{H}\left(\partial^{e}(E \cap F) \cap \partial^{e}(E \backslash F)\right) & \leq \mathcal{H}\left(\partial^{e}(E \cap F) \backslash \partial^{e} E\right)=\mathcal{H}\left(\partial^{e} F \backslash \partial^{e} E\right)=0, \\
\mathcal{H}\left(\partial^{e}\left(E^{c} \cap F\right) \cap \partial^{e}\left(E^{c} \backslash F\right)\right) & \leq \mathcal{H}\left(\partial^{e}\left(E^{c} \cap F\right) \backslash \partial^{e} E^{c}\right)=\mathcal{H}\left(\partial^{e} F \backslash \partial^{e} E\right)=0 .
\end{aligned}
$$

Then item (ii) of Lemma 2.3 yields $\mathrm{P}(E)=\mathrm{P}(E \cap F)+\mathrm{P}(E \backslash F)$ and $\mathrm{P}\left(E^{c}\right)=\mathrm{P}\left(E^{c} \cap F\right)+$ $\mathrm{P}\left(E^{c} \backslash F\right)$. Being $E$ (resp. $\left.E^{c}\right)$ indecomposable, we conclude that either $\mathfrak{m}(E \cap F)=0$ or $\mathfrak{m}(E \backslash F)=0$ (resp. either $\mathfrak{m}\left(E^{c} \cap F\right)=0$ or $\mathfrak{m}\left(E^{c} \backslash F\right)=0$ ). This implies that $\mathfrak{m}(F)=0$, $\mathfrak{m}\left(F^{c}\right)=0, \mathfrak{m}(F \Delta E)=0$ or $\mathfrak{m}\left(F \Delta E^{c}\right)=0$, thus proving that $E$ is a simple set.

Remark 3.3 In item (ii) of Proposition 3.2, the additional assumption on the space cannot be dropped. To show it, let us consider the closed unit ball $\mathrm{X}_{1}$ centered at $o$ of the 3 -spider $\left(S_{3}, d, \mathfrak{m}\right)$. We claim that the conclusion of item (ii) of Proposition 3.2 fails in $\left(\mathrm{X}_{1}, d_{\mathrm{X}_{1} \times \mathrm{X}_{1}, \mathfrak{m}\left\llcorner\mathrm{X}_{1}\right.}\right)$.

By Example 1.29 we know that the two-sidedness property is not satisfied. Now call $R_{1}^{\prime}, R_{2}^{\prime}, R_{3}^{\prime}$ the intersections of the rays of $S_{3}$ with $\mathrm{X}_{1}$. It thus holds that $R_{1}^{\prime} \cup R_{2}^{\prime}$ and $\mathrm{X}_{1} \backslash\left(R_{1}^{\prime} \cup\right.$ $\left.R_{2}^{\prime}\right)=R_{3}^{\prime} \cup\{o\}$ are indecomposable, but the set $R_{1}^{\prime} \cup R_{2}^{\prime}$ is not simple.

Let $(\mathrm{X}, d, \mathfrak{m})$ be a metric measure space. Let $K \subset \mathrm{X}$ be a compact set. Then we define

$$
\mathcal{K}(\mathrm{X} ; K):=\{f \in \mathrm{BV}(\mathrm{X})|\operatorname{spt}(f) \subset K,| D f \mid(\mathrm{X}) \leq 1\} .
$$

Remark 3.4 It holds that

$$
\mathcal{K}(\mathrm{X} ; K) \text { is a convex, compact subset of } L^{1}(\mathfrak{m}) .
$$

First of all, its convexity is granted by item (ii) of Proposition 1.2. To prove compactness, fix any sequence $\left(f_{n}\right)_{n} \subset \mathcal{K}(\mathrm{X} ; K)$. Item (iii) of Proposition 1.2 says that $f_{n_{i}} \rightarrow f$ in $L_{\text {loc }}^{1}(\mathrm{X})$ for some subsequence $\left(n_{i}\right)_{i}$ and some limit function $f \in L_{\text {loc }}^{1}(\mathfrak{m})$. Given that $\operatorname{spt}\left(f_{n}\right) \subset K$ for every $n \in \mathbb{N}$, we know that $\operatorname{spt}(f) \subset K$, thus $f \in L^{1}(\mathfrak{m})$ and $f_{n_{i}} \rightarrow f$ in $L^{1}(\mathfrak{m})$. Finally, by using item (i) of Proposition 1.2 we conclude that $|D f|(\mathrm{X}) \leq \underline{\lim }_{i}\left|D f_{n_{i}}\right|(\mathrm{X}) \leq 1$, whence $f \in \mathcal{K}(\mathrm{X} ; K)$.

Recall that ext $\mathcal{K}(\mathrm{X} ; K)$ stands for the set of all extreme points of $\mathcal{K}(\mathrm{X} ; K)$; cf. "Appendix A". Furthermore, observe that $|D f|(\mathrm{X})=1$ holds for every $f \in \operatorname{ext} \mathcal{K}(\mathrm{X} ; K)$. In the remaining 
part of this subsection, we shall study in detail the family ext $\mathcal{K}(\mathrm{X} ; K)$. Our arguments are strongly inspired by the ideas of the papers [23,24].

Let $(\mathrm{X}, d, \mathfrak{m})$ be a PI space. Given a set $E \subset \mathrm{X}$ of finite perimeter satisfying $0<\mathfrak{m}(E)<$ $+\infty$ and $\mathfrak{m}\left(E^{c}\right)>0$ (so that $\mathrm{P}(E)>0$ ), let us define

$$
\Phi_{ \pm}(E):= \pm \frac{\mathbb{1}_{E}}{\mathrm{P}(E)} \in \mathrm{BV}(\mathrm{X}) .
$$

Observe that $\left|D \Phi_{+}(E)\right|(\mathrm{X})=\left|D \Phi_{-}(E)\right|(\mathrm{X})=1$. For any compact set $K \subsetneq \mathrm{X}$, we define

$$
\begin{aligned}
& \mathcal{F}(\mathrm{X} ; K):=\left\{\Phi_{ \pm}(E) \mid E \subset K \text { is a set of finite perimeter with } \mathfrak{m}(E)>0\right\}, \\
& \mathcal{I}(\mathrm{X} ; K):=\left\{\Phi_{ \pm}(E) \mid E \subset K \text { is an indecomposable set with } \mathfrak{m}(E)>0\right\} \\
& \mathcal{S}(\mathrm{X} ; K):=\left\{\Phi_{ \pm}(E) \mid E \subset K \text { is a simple set with } \mathfrak{m}(E)>0\right\} .
\end{aligned}
$$

Observe that $\mathcal{S}(\mathrm{X} ; K), \mathcal{I}(\mathrm{X} ; K) \subset \mathcal{F}(\mathrm{X} ; K) \subset \mathcal{K}(\mathrm{X} ; K)$. Given any function $f \in \mathcal{F}(\mathrm{X} ; K)$, we shall denote by $E_{f} \subset \mathrm{X}$ the (m-a.e. unique) Borel set satisfying either $f=\Phi_{+}\left(E_{f}\right)$ or $f=\Phi_{-}\left(E_{f}\right)$. If, in addition, the space $(\mathrm{X}, d, \mathfrak{m})$ is isotropic, then $\mathcal{S}(\mathrm{X} ; K) \subset \mathcal{I}(\mathrm{X} ; K)$ by item (i) of Proposition 3.2.

Proposition 3.5 Let $(\mathrm{X}, d, \mathfrak{m})$ be a PI space and $K \subsetneq \mathrm{X}$ a compact set. Then the closed convex hull of the set $\mathcal{F}(\mathrm{X} ; K)$ coincides with $\mathcal{K}(\mathrm{X} ; K)$.

Proof We aim to show that any function $f \in \mathcal{K}(\mathrm{X} ; K)$ can be approximated in $L^{1}(\mathfrak{m})$ by convex combinations of elements in $\mathcal{F}(\mathrm{X} ; K)$. Let us apply Lemma 1.21: we can find a sequence $\left(f_{n}\right)_{n}$ of simple BV functions supported on the set $K$, say $f_{n}=\sum_{i=1}^{k_{n}} \lambda_{i}^{n} \mathbb{1}_{E_{i}^{n}}$, so that $f_{n} \rightarrow f$ in $L^{1}(\mathfrak{m})$ and $\sum_{i=1}^{k_{n}}\left|\lambda_{i}^{n}\right| \mathrm{P}\left(E_{i}^{n}\right) \leq 1$ (recall Remark 1.22). Given that we have $\Phi_{\operatorname{sgn}\left(\lambda_{i}^{n}\right)}\left(E_{i}^{n}\right) \in \mathcal{F}(\mathrm{X} ; K)$ and

$$
\frac{f_{n}}{q}=\sum_{i=1}^{k_{n}} \frac{\left|\lambda_{i}^{n}\right| \mathrm{P}\left(E_{i}^{n}\right)}{q} \Phi_{\operatorname{sgn}\left(\lambda_{i}^{n}\right)}\left(E_{i}^{n}\right), \quad \text { where we set } q:=\sum_{i=1}^{k_{n}}\left|\lambda_{i}^{n}\right| \mathrm{P}\left(E_{i}^{n}\right) \in[0,1],
$$

we conclude that the functions $f_{n} / q$ belong to the convex hull of $\mathcal{F}(\mathrm{X} ; K)$. Given that $\mathcal{F}(\mathrm{X} ; K)$ is symmetric, we know that its convex hull contains the function 0 and accordingly also all the functions $f_{n}$. The statement follows.

Lemma 3.6 Let $(\mathrm{X}, d, \mathfrak{m})$ be a PI space and let $K \subsetneq \mathrm{X}$ be a compact set. Then it holds that

$$
\{\lambda f \mid \lambda \in[-1,1], f \in \mathcal{F}(\mathrm{X} ; K)\} \quad \text { is strongly closed in } L^{1}(\mathfrak{m}) \text {. }
$$

Proof Let us call $\mathcal{B}:=\{\lambda f: \lambda \in[-1,1], f \in \mathcal{F}(\mathrm{X} ; K)\}$. Fix a sequence $\left(f_{n}\right)_{n} \subset \mathcal{B}$ converging to some function $f \in L^{1}(\mathfrak{m})$ in $L^{1}(\mathfrak{m})$. We aim to show that $f \in \mathcal{B}$ as well. Given any $n \in \mathbb{N}$, we can find $\lambda_{n} \in[-1,1]$ and a set of finite perimeter $E_{n} \subset K$ such that $\mathfrak{m}\left(E_{n}\right)>0$ and $f_{n}=\lambda_{n} \Phi_{+}\left(E_{n}\right)$. We subdivide the proof into three different cases:

(1) Suppose $\lim _{n} \mathrm{P}\left(E_{n}\right)=0$. Then there exists a set of finite perimeter $E \subset K$ such that (up to a not relabelled subsequence) it holds $\mathbb{1}_{E_{n}} \rightarrow \mathbb{1}_{E}$ in $L^{1}(\mathfrak{m})$. In particular, $\mathrm{P}(E) \leq \lim _{n} \mathrm{P}\left(E_{n}\right)=0$ and accordingly $\lim _{n} \mathfrak{m}\left(E_{n}\right)=\mathfrak{m}(E)=0$. Possibly passing to a further subsequence, we may thus assume that $\mathfrak{m}\left(E_{n}\right)<1 / 2^{n}$ for all $n \in \mathbb{N}$. Since the identity $f_{k}(x)=0$ holds for every $k \geq n>0$ and $x \in K \backslash \bigcup_{m \geq n} E_{m}$, we deduce that $\lim _{k} f_{k}(x)=0$ for every $x \in K \backslash \bigcap_{n} \bigcup_{m \geq n} E_{m}$. This implies that $f=0 \in \mathcal{B}$, as the set $\bigcap_{n} \bigcup_{m \geq n} E_{m}$ is m-negligible by Borel-Cantelli lemma. 
(2) Suppose that $\lim _{n} \mathrm{P}\left(E_{n}\right)=+\infty$. Then it holds that

$$
\varlimsup_{n \rightarrow \infty} \int\left|f_{n}\right| \mathrm{dm} \leq \varlimsup_{n \rightarrow \infty} \frac{\left|\lambda_{n}\right| \mathfrak{m}\left(E_{n}\right)}{\mathrm{P}\left(E_{n}\right)} \leq \varlimsup_{n \rightarrow \infty} \frac{\mathfrak{m}(K)}{\mathrm{P}\left(E_{n}\right)}=0,
$$

whence accordingly $f=0 \in \mathcal{B}$.

(3) Suppose $\underline{\lim }_{n} \mathrm{P}\left(E_{n}\right)>0$ and $\varlimsup_{n} \mathrm{P}\left(E_{n}\right)<+\infty$. Then there exist $\lambda \in[-1,1]$ and $c \in(0,+\infty)$ such that - up to a not relabelled subsequence-one has that $\lambda_{n} \rightarrow \lambda$ and $\lim _{n} \mathrm{P}\left(E_{n}\right)=c$. We can further assume that $\mathbb{1}_{E_{n}} \rightarrow \mathbb{1}_{E}$ strongly in $L^{1}(\mathfrak{m})$, for some set of finite perimeter $E \subset K$. Therefore, we deduce that $f=\lambda \mathbb{1}_{E} / c$. If $\mathfrak{m}(E)=0$, then $f=0 \in \mathcal{B}$. If $\mathfrak{m}(E)>0$, then we can write $f$ as $\lambda^{\prime} \Phi_{+}(E)$, where we set $\lambda^{\prime}:=\lambda \mathrm{P}(E) / c$. Since $\mathrm{P}(E) \leq \lim _{n} \mathrm{P}\left(E_{n}\right)=c$ by lower semicontinuity of the perimeter, we conclude that $\lambda^{\prime} \in[-1,1]$ and accordingly $f \in \mathcal{B}$.

Theorem 3.7 Let $(\mathrm{X}, d, \mathfrak{m})$ be a PI space and let $K \subsetneq \mathrm{X}$ be a compact set. Then it holds that

$$
\operatorname{ext} \mathcal{K}(\mathrm{X} ; K) \subset \mathcal{I}(\mathrm{X} ; K) \text {. }
$$

Proof By Milman Theorem A.1, Proposition 3.5, and Lemma 3.6, we know that any extreme point of $\mathcal{K}(\mathrm{X} ; K)$ can be written as $\lambda f$ for some $\lambda \in[-1,1]$ and $f \in \mathcal{F}(\mathrm{X} ; K)$. Moreover, it is clear that $\lambda f \notin \operatorname{ext} \mathcal{K}(\mathrm{X} ; K)$ for every $\lambda \in(-1,1)$ and $f \in \mathcal{F}(\mathrm{X} ; K)$, since $\lambda f=$ $\frac{1+\lambda}{2} f+\frac{1-\lambda}{2}(-f)$. This shows that ext $\mathcal{K}(\mathrm{X} ; K) \subset \mathcal{F}(\mathrm{X} ; K)$. It only remains to prove that if $f=\Phi_{\sigma}(E) \in \operatorname{ext} \mathcal{K}(\mathrm{X} ; K)$, then $E$ is indecomposable. We argue by contradiction: suppose the set $E$ is decomposable, so that there exist disjoint Borel sets $F, G \subset E$ such that $\mathfrak{m}(F), \mathfrak{m}(G)>0$ and $\mathrm{P}(E)=\mathrm{P}(F)+\mathrm{P}(G)$. Therefore, we can write

$$
\Phi_{\sigma}(E)=\frac{\mathrm{P}(F)}{\mathrm{P}(E)} \Phi_{\sigma}(F)+\frac{\mathrm{P}(G)}{\mathrm{P}(E)} \Phi_{\sigma}(G) .
$$

This contradicts the fact that $f$ is an extreme point of $\mathcal{K}(\mathrm{X} ; K)$, thus the set $E$ is proven to be indecomposable. Hence, we have that ext $\mathcal{K}(\mathrm{X} ; K) \subset \mathcal{I}(\mathrm{X} ; K)$, as required.

Theorem 3.8 Let $(\mathrm{X}, d, \mathfrak{m})$ be an isotropic PI space. Let $K \subsetneq \mathrm{X}$ be a compact set. Then:

(i) It holds that

$$
\mathcal{S}(\mathrm{X} ; K) \subset \operatorname{ext} \mathcal{K}(\mathrm{X} ; K) \text {. }
$$

(ii) Suppose that $(\mathrm{X}, d, \mathfrak{m})$ has the two-sidedness property. Suppose also that $K$ has finite perimeter, that $\mathcal{H}\left(\partial K \backslash \partial^{e} K\right)=0$ and that $K^{c}$ is connected. Then $\mathcal{S}(\mathrm{X} ; K)=$ ext $\mathcal{K}(\mathrm{X} ; K)$.

Proof (i) Let $f \in \mathcal{S}(\mathrm{X} ; K)$ be fixed. Thanks to Choquet Theorem A.2, there exists a Borel probability measure $\mu$ on $L^{1}(\mathfrak{m})$, concentrated on ext $\mathcal{K}(\mathrm{X} ; K)$, such that

$$
\int f \varphi \mathrm{d} \mathfrak{m}=\iint g \varphi \mathrm{d} \mathfrak{m} \mathrm{d} \mu(g) \quad \text { for every } \varphi \in L^{\infty}(\mathfrak{m}) .
$$

We claim that, given any Borel set $B \subset \mathrm{X}$, the functional $\Psi_{B}: L^{1}(\mathfrak{m}) \rightarrow[0,+\infty)$ given by

$$
\Psi_{B}(g):=\mathbb{1}_{\mathrm{BV}(\mathrm{X})}(g)|D g|(B) \quad \text { for every } g \in L^{1}(\mathfrak{m})
$$

is Borel measurable. To prove it, call $\mathcal{D}$ the family of all Borel sets $B \subset \mathrm{X}$ such that $\Psi_{B}$ is a Borel measurable function. Observe that: 
(a) $\mathrm{X} \in \mathcal{D}$ by item (i) of Proposition 1.2.

(b) If $A, B \in \mathcal{D}$ satisfy $A \subset B$, then $|D g|(B \backslash A)=|D g|(B)-|D g|(A)$ for all $g \in \mathrm{BV}(\mathrm{X})$. This implies that $\Psi_{B \backslash A}=\Psi_{B}-\Psi_{A}$ is Borel measurable and thus $B \backslash A \in \mathcal{D}$.

(c) Given any increasing sequence $\left(A_{n}\right)_{n} \subset \mathcal{D}$, we have that $|D g|\left(\bigcup_{n} A_{n}\right)=$ $\lim _{n}|D g|\left(A_{n}\right)$ for all $g \in L^{1}(\mathfrak{m})$ thanks to the continuity from below, whence accordingly $\Psi_{\cup_{n} A_{n}}$ is Borel measurable (so that $\bigcup_{n} A_{n} \in \mathcal{D}$ ) as it is the pointwise limit of $\Phi_{A_{n}}$ as $n \rightarrow \infty$.

All in all, we have proven that $\mathcal{D}$ is a Dynkin system. Given that the topology of $(\mathrm{X}, d)$ is contained in $\mathcal{D}$ (again by item (i) of Proposition 1.2), we conclude that $\mathcal{D}$ coincides with the Borel $\sigma$-algebra of X by the Dynkin $\pi-\lambda$ Theorem. This proves that $\Psi_{B}$ is Borel for any $B \subset \mathrm{X}$ Borel, as claimed.

With this said, it makes sense to define the Borel measure $v$ on $\mathrm{X}$ as $v:=\int|D g| \mathrm{d} \mu(g)$, namely

$$
v(B)=\int|D g|(B) \mathrm{d} \mu(g) \quad \text { for every Borel set } B \subset \mathrm{X} .
$$

Given that $|D g|(\mathrm{X})=1$ for every $g \in \operatorname{ext} \mathcal{K}(\mathrm{X} ; K)$, we know that $|D g|(\mathrm{X})=1$ for $\mu$-a.e. $g \in$ $L^{1}(\mathfrak{m})$ and accordingly $v$ is a probability measure. Now fix any open set $\Omega \subset \mathrm{X}$ containing $\partial^{e} E_{f}$. Thanks to Theorem 1.4, we can find a sequence of derivations $\left(\boldsymbol{b}_{n}\right)_{n} \subset \operatorname{Der}_{\mathrm{b}}(\mathrm{X})$ such that $\left|\boldsymbol{b}_{n}\right| \leq 1$ in the $\mathfrak{m}$-a.e. sense, $\operatorname{spt}\left(\boldsymbol{b}_{n}\right) \Subset \Omega$ and $\int_{\Omega} f \operatorname{div}\left(\boldsymbol{b}_{n}\right) \mathrm{dm} \rightarrow|D f|(\Omega)$. Therefore, it holds that

$$
|D f|(\Omega) \stackrel{(3.2)}{=} \lim _{n \rightarrow \infty} \iint_{\Omega} g \operatorname{div}\left(\boldsymbol{b}_{n}\right) \mathrm{d} \mathfrak{m} \mathrm{d} \mu(g) \leq \int|D g|(\Omega) \mathrm{d} \mu(g)=v(\Omega) .
$$

Given that $|D f|$ and $v$ are outer regular, we can pick a sequence $\left(\Omega_{n}\right)_{n}$ of open subsets of X containing $\partial^{e} E_{f}$ such that $|D f|\left(\partial^{e} E_{f}\right)=\lim _{n}|D f|\left(\Omega_{n}\right)$ and $v\left(\partial^{e} E_{f}\right)=\lim _{n} v\left(\Omega_{n}\right)$. By recalling the inequality (3.3), we thus obtain that

$$
1=\frac{\mathrm{P}\left(E_{f}, \partial^{e} E_{f}\right)}{\mathrm{P}\left(E_{f}\right)}=|D f|\left(\partial^{e} E_{f}\right)=\lim _{n \rightarrow \infty}|D f|\left(\Omega_{n}\right) \leq \varliminf_{n \rightarrow \infty} v\left(\Omega_{n}\right)=v\left(\partial^{e} E_{f}\right)=1 .
$$

This forces the equality $\int|D g|\left(\partial^{e} E_{f}\right) \mathrm{d} \mu(g)=v\left(\partial^{e} E_{f}\right)=1$. Given that $|D g|\left(\partial^{e} E_{f}\right) \leq 1$ holds for $\mu$-a.e. $g \in L^{1}(\mathfrak{m})$, we infer that actually $|D g|\left(\partial^{e} E_{f}\right)=1$ for $\mu$-a.e. $g \in L^{1}(\mathfrak{m})$. Since $g \in \mathcal{I}(X ; K)$ for $\mu$-a.e. $g \in L^{1}(\mathfrak{m})$ by Theorem 3.7, it makes sense to consider $E_{g}$ for $\mu$-a.e. $g \in L^{1}(\mathfrak{m})$. Therefore, we have that

$$
\begin{aligned}
(\theta \mathcal{H})\left(\partial^{e} E_{g} \backslash \partial^{e} E_{f}\right) & =\mathrm{P}\left(E_{g},\left(\partial^{e} E_{f}\right)^{c}\right)=\mathrm{P}\left(E_{g}\right)\left(1-\frac{\mathrm{P}\left(E_{g}, \partial^{e} E_{f}\right)}{\mathrm{P}\left(E_{g}\right)}\right) \\
& =\mathrm{P}\left(E_{g}\right)\left(1-|D g|\left(\partial^{e} E_{f}\right)\right)=0
\end{aligned}
$$

holds for $\mu$-a.e. $g \in L^{1}(\mathfrak{m})$. This implies that $\mathcal{H}\left(\partial^{e} E_{g} \backslash \partial^{e} E_{f}\right)=0$ for $\mu$-a.e. $g \in L^{1}(\mathfrak{m})$. Since $E_{f}$ is a simple set, we deduce that $\mathfrak{m}\left(E_{g} \Delta E_{f}\right)=0$ for $\mu$-a.e. $g \in L^{1}(\mathfrak{m})$. This forces $\mu=t \delta_{f}+(1-t) \delta_{-f}$ for some $t \in[0,1]$. Given that $\mu$ is concentrated on the symmetric set ext $\mathcal{K}(\mathrm{X} ; K)$, we finally conclude that $f \in \operatorname{ext} \mathcal{K}(\mathrm{X} ; K)$, as required. This proves the inclusion (3.1).

(ii) Let $f \in \operatorname{ext} \mathcal{K}(\mathrm{X} ; K)$ be fixed. Take a set $F \subset \mathrm{X}$ of finite perimeter with $\mathcal{H}\left(\partial^{e} F \backslash \partial^{e} E_{f}\right)=0$. We claim that either $\mathfrak{m}(F \backslash K)=0$ or $\mathfrak{m}\left(F^{c} \backslash K\right)=0$. To prove it, notice that (1.7), (1.18) give

$$
\begin{aligned}
\mathcal{H}\left(\partial^{e}(F \backslash K) \cap \partial^{e}\left(F^{c} \backslash K\right)\right) & \leq \mathcal{H}\left(\left(\partial^{e} F \cup \partial^{e} K\right) \backslash K\right)+\mathcal{H}\left(\partial^{e}(F \backslash K) \cap \partial^{e}\left(F^{c} \backslash K\right) \cap \partial K\right) \\
& \leq \mathcal{H}\left(\partial^{e} F \backslash \partial^{e} E_{f}\right)+\mathcal{H}\left(\partial^{e}(F \backslash K) \cap \partial^{e}\left(F^{c} \backslash K\right) \cap \partial^{e} K\right)=0 .
\end{aligned}
$$


Accordingly, item (ii) of Lemma 2.3 yields $\mathrm{P}\left(K^{c}\right)=\mathrm{P}(F \backslash K)+\mathrm{P}\left(F^{c} \backslash K\right)$. Being $K^{c}$ indecomposable by Corollary 2.7, we conclude that either $\mathfrak{m}(F \backslash K)=0$ or $\mathfrak{m}\left(F^{c} \backslash K\right)=0$, as desired. Now call

$$
G:=\left\{\begin{array}{lll}
F & \text { if } & \mathfrak{m}(F \backslash K)=0, \\
F^{c} & \text { if } & \mathfrak{m}\left(F^{c} \backslash K\right)=0 .
\end{array}\right.
$$

We aim to prove that either $\mathfrak{m}(G)=0$ or $\mathfrak{m}\left(G \Delta E_{f}\right)=0$. Suppose that $\mathfrak{m}(G)>0$. Observe that

$$
\mathbb{1}_{E_{f}}=\mathbb{1}_{G \cup E_{f}}-\mathbb{1}_{G \backslash E_{f}}=\mathbb{1}_{E_{f} \cap G}+\mathbb{1}_{E_{f} \backslash G} .
$$

Thanks to the two-sidedness property, we also know that

$$
\mathcal{H}\left(\partial^{e}\left(G \cup E_{f}\right) \cap \partial^{e}\left(G \backslash E_{f}\right) \cap \partial^{e} E_{f}^{c}\right)=0=\mathcal{H}\left(\partial^{e}\left(E_{f} \cap G\right) \cap \partial^{e}\left(E_{f} \backslash G\right) \cap \partial^{e} E_{f}\right) .
$$

Therefore, item (ii) of Lemma 2.3 grants that

$$
\begin{aligned}
& \mathrm{P}\left(E_{f}\right)=\mathrm{P}\left(E_{f}^{c}, \partial^{e} E_{f}^{c}\right)=\mathrm{P}\left(G \cup E_{f}, \partial^{e} E_{f}\right)+\mathrm{P}\left(G \backslash E_{f}, \partial^{e} E_{f}\right)=\mathrm{P}\left(G \cup E_{f}\right)+\mathrm{P}\left(G \backslash E_{f}\right), \\
& \mathrm{P}\left(E_{f}\right)=\mathrm{P}\left(E_{f}, \partial^{e} E_{f}\right)=\mathrm{P}\left(E_{f} \cap G, \partial^{e} E_{f}\right)+\mathrm{P}\left(E_{f} \backslash G, \partial^{e} E_{f}\right)=\mathrm{P}\left(E_{f} \cap G\right)+\mathrm{P}\left(E_{f} \backslash G\right) .
\end{aligned}
$$

Suppose by contradiction that $\mathfrak{m}\left(G \backslash E_{f}\right)>0$. Then we have $\mathrm{P}\left(G \backslash E_{f}\right)>0$ and accordingly

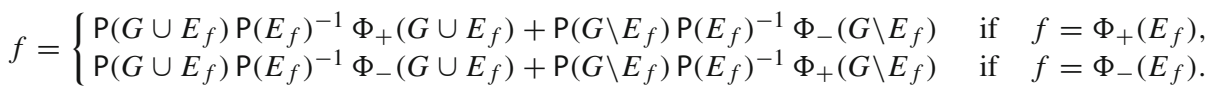

This contradicts the fact that $f \in \operatorname{ext} \mathcal{K}(\mathrm{X} ; K)$, whence $\mathfrak{m}\left(G \backslash E_{f}\right)=0$. Similarly, suppose by contradiction that $\mathfrak{m}\left(E_{f} \backslash G\right)>0$. Then we have $\mathrm{P}\left(E_{f} \backslash G\right)>0$ and accordingly $f=\left\{\begin{array}{l}\mathrm{P}\left(E_{f} \cap G\right) \mathrm{P}\left(E_{f}\right)^{-1} \Phi_{+}\left(E_{f} \cap G\right)+\mathrm{P}\left(E_{f} \backslash G\right) \mathrm{P}\left(E_{f}\right)^{-1} \Phi_{+}\left(E_{f} \backslash G\right) \\ \mathrm{P}\left(E_{f} \cap G\right) \mathrm{P}\left(E_{f}\right)^{-1} \Phi_{-}\left(E_{f} \cap G\right)+\mathrm{P}\left(E_{f} \backslash G\right) \mathrm{P}\left(E_{f}\right)^{-1} \Phi_{-}\left(E_{f} \backslash G\right)\end{array}\right.$

This contradicts the fact that $f \in \operatorname{ext} \mathcal{K}(\mathrm{X} ; K)$, whence $\mathfrak{m}\left(E_{f} \backslash G\right)=0$. This yields $\mathfrak{m}\left(G \Delta E_{f}\right)=0$, thus the set $E_{f}$ is proven to be simple. We conclude that $f \in \mathcal{S}(\mathrm{X} ; K)$, as required.

\subsection{Holes and saturation}

The decomposition theorem can be used to define suitable notions of hole and saturation for a given set of finite perimeter in an isotropic PI space:

Definition 3.9 (Hole) Let $(\mathrm{X}, d, \mathfrak{m})$ be an isotropic PI space such that $\mathfrak{m}(\mathrm{X})=+\infty$. Let $E \subset \mathrm{X}$ be an indecomposable set. Then any essential connected component of $\mathrm{X} \backslash E$ having finite $\mathfrak{m}$-measure is said to be a hole of $E$.

Definition 3.10 (Saturation) Let $(\mathrm{X}, d, \mathfrak{m})$ be an isotropic PI space such that $\mathfrak{m}(\mathrm{X})=+\infty$. Given an indecomposable set $F \subset \mathrm{X}$, we define its saturation $\operatorname{sat}(F)$ as the union of $F$ and its holes. Moreover, given any set $E \subset \mathrm{X}$ of finite perimeter, we define

$$
\operatorname{sat}(E):=\bigcup_{F \in \mathcal{C C}^{e}(E)} \operatorname{sat}(F)
$$

We say that the set $E$ is saturated provided it holds that $\mathfrak{m}(E \Delta \operatorname{sat}(E))=0$.

Observe that an indecomposable set $E \subset \mathrm{X}$ is saturated if and only if it has no holes. 
Remark 3.11 Given an isotropic PI space $(\mathrm{X}, d, \mathfrak{m})$ such that $\mathfrak{m}(\mathrm{X})=+\infty$, it holds that any simple set $E \subset \mathrm{X}$ is indecomposable and saturated. Indeed, item (i) of Proposition 3.2 grants that both $E, E^{c}$ are indecomposable; since $\mathfrak{m}\left(E^{c}\right)=+\infty$, we also conclude that $E$ has no holes.

Proposition 3.12 (Main properties of the saturation) Let $(\mathrm{X}, d, \mathfrak{m})$ be an isotropic PI space such that $\mathfrak{m}(\mathrm{X})=+\infty$. Let $E \subset \mathrm{X}$ be an indecomposable set. Then the following properties hold:

(i) Any hole of $E$ is saturated.

(ii) The set $\operatorname{sat}(E)$ is indecomposable and saturated. In particular, $\operatorname{sat}(\operatorname{sat}(E))=\operatorname{sat}(E)$.

(iii) It holds that $\mathcal{H}\left(\partial^{e} \operatorname{sat}(E) \backslash \partial^{e} E\right)=0$. In particular, one has that $P(\operatorname{sat}(E)) \leq P(E)$.

(iv) If $F \subset \mathrm{X}$ is a set of finite perimeter with $\mathfrak{m}(E \backslash \operatorname{sat}(F))=0$, then $\mathfrak{m}(\operatorname{sat}(E) \backslash \operatorname{sat}(F))=0$.

\section{Proof}

(i) Let $F$ be a hole of $E$. Denote $\mathcal{C} \mathcal{C}^{e}\left(E^{c}\right)=\{F\} \cup\left\{G_{i}\right\}_{i \in I}$. We know from Remark 2.17 that $\mathcal{H}\left(\partial^{e} G_{i} \cap \partial^{e} E\right)=\mathcal{H}\left(\partial^{e} G_{i}\right)>0$ for all $i \in I$, thus $E \cup \bigcup_{i \in J} G_{i}$ is indecomposable for any finite set $J \subset I$ by Proposition 2.18. Therefore, the set $F^{c}=E \cup \bigcup_{i \in I} G_{i}$ is indecomposable by Proposition 2.10. Given that $\mathfrak{m}\left(F^{c}\right)=+\infty$, we conclude that $F$ has no holes, as required.

(ii) Let us call $\left\{F_{i}\right\}_{i \in I}$ the holes of $E$. By arguing exactly as in the proof of item (i), we see that the set $\operatorname{sat}(E)=E \cup \bigcup_{i \in I} F_{i}$ is indecomposable. Moreover, $\mathcal{C} C^{e}\left(\operatorname{sat}(E)^{c}\right)=$ $\mathcal{C C}^{e}\left(E^{c}\right) \backslash\left\{F_{i}\right\}_{i \in I}$, so that sat $(E)$ has no holes. In other words, the set sat $(E)$ is saturated.

(iii) Calling $\left\{F_{i}\right\}_{i \in I}$ the holes of $E$, we clearly have that $\partial^{e} \operatorname{sat}(E) \subset \partial^{e} E \cup \bigcup_{i \in I} \partial^{e} F_{i}$ by (1.7). Given that $\mathcal{H}\left(\partial^{e} F_{i} \backslash \partial^{e} E\right)=0$ for all $i \in I$ by Remark 2.17, we conclude that $\mathcal{H}\left(\partial^{e} \operatorname{sat}(E) \backslash \partial^{e} E\right)=0$ as well. Furthermore, observe that the latter identity also yields

$$
\mathrm{P}(\operatorname{sat}(E))=\left(\theta_{\operatorname{sat}(E)} \mathcal{H}\right)\left(\partial^{e} \operatorname{sat}(E)\right) \stackrel{(1.15)}{=}\left(\theta_{E} \mathcal{H}\right)\left(\partial^{e} \operatorname{sat}(E)\right) \leq\left(\theta_{E} \mathcal{H}\right)\left(\partial^{e} E\right)=\mathrm{P}(E) .
$$

(iv) Let us denote $\mathcal{C C}^{e}\left(\operatorname{sat}(F)^{c}\right)=\left\{F_{i}\right\}_{i \in I}$. Given any $i \in I$, we have that $F_{i}$ is indecomposable, has infinite $\mathfrak{m}$-measure, and satisfies $\mathfrak{m}\left(E \cap F_{i}\right)=0$. Then there exists a unique set $G_{i} \in \mathcal{C} C^{e}\left(E^{c}\right)$ such that $\mathfrak{m}\left(F_{i} \backslash G_{i}\right)=0$, thus in particular $\mathfrak{m}\left(G_{i}\right)=+\infty$. This says that the sets $\left\{G_{i}\right\}_{i \in I}$ cannot be holes of $E$, whence $\bigcup_{i \in I} G_{i} \subset \operatorname{sat}(E)^{c}$ and accordingly $\mathfrak{m}(\operatorname{sat}(E) \backslash \operatorname{sat}(F))=0$.

Lemma 3.13 Let $(\mathrm{X}, d, \mathfrak{m})$ be an isotropic PI space such that $\mathfrak{m}(\mathrm{X})=+\infty$. Let $E \subset \mathrm{X}$ be a set of finite perimeter. Then it holds that $\mathcal{H}\left(\partial^{e} \operatorname{sat}(E) \backslash \partial^{e} E\right)=0$.

Proof Given any $F \in \mathcal{C C}^{e}(E)$, we have $\mathcal{H}\left(\partial^{e}\right.$ sat $\left.(F) \backslash \partial^{e} F\right)=0$ by item (iii) of Proposition 3.12. Moreover, since $\operatorname{sat}(E)=\bigcup_{F \in \mathcal{C} C^{e}(E)} \operatorname{sat}(F)$ we know that $\partial^{e} \operatorname{sat}(E) \subset$ $\bigcup_{F \in \mathcal{C} C^{e}(E)} \partial^{e} \operatorname{sat}(F)$ as a consequence of (1.7). Therefore, we deduce that

$$
\mathcal{H}\left(\partial^{e} \operatorname{sat}(E) \backslash \partial^{e} E\right) \leq \sum_{F \in \mathcal{C} \mathcal{C}^{e}(E)} \mathcal{H}\left(\partial^{e} \operatorname{sat}(F) \backslash \partial^{e} E\right) \leq \sum_{F \in \mathcal{C} \mathcal{C}^{e}(E)} \mathcal{H}\left(\partial^{e} F \backslash \partial^{e} E\right) \stackrel{(2.16)}{=} 0,
$$

thus proving the statement.

Let us now focus on the special case of Ahlfors-regular, isotropic PI spaces. In this context, simple sets can be equivalently characterised as those sets that are both indecomposable and saturated (cf. Theorem 3.17). In order to prove it, we need some preliminary results: 
Proposition 3.14 Let $(\mathrm{X}, d, \mathfrak{m})$ be a $k$-Ahlfors regular, isotropic PI space with $k>1$. Suppose that $\mathfrak{m}(\mathrm{X})=+\infty$. Let $E \subset \mathrm{X}$ be an indecomposable set such that $\mathfrak{m}(E)<+\infty$. Then there exists exactly one essential connected component $F \in \mathcal{C C}^{e}\left(E^{c}\right)$ satisfying $\mathfrak{m}(F)=+\infty$.

Proof Let us prove that at least one essential connected component of $E^{c}$ has infinite $\mathfrak{m}$ measure. We argue by contradiction: suppose $\mathfrak{m}\left(E_{i}\right)<+\infty$ for all $i \in I$, where we set $\mathcal{C C}^{e}(E)=\left\{E_{i}\right\}_{i \in I}$. In particular, we have that $\mathfrak{m}\left(E_{i}^{c}\right)=+\infty$ holds for every $i \in I$, whence Theorem 1.18 yields

$$
\sum_{i \in I} \mathfrak{m}\left(E_{i}\right)^{k-1 / k} \leq C_{I}^{\prime} \sum_{i \in I} \mathrm{P}\left(E_{i}\right)=C_{I}^{\prime} \mathrm{P}(E)<+\infty .
$$

By using the Markov inequality we deduce that $J:=\left\{i \in I: \mathfrak{m}\left(E_{i}\right) \geq 1\right\}$ is a finite family, thus the set $\bigcup_{i \in J} E_{i}$ has finite $\mathfrak{m}$-measure. This leads to a contradiction, as it implies that

$$
+\infty=\mathfrak{m}\left(\bigcup_{i \in I \backslash J} E_{i}\right)=\sum_{i \in I \backslash J} \mathfrak{m}\left(E_{i}\right) \leq \sum_{i \in I \backslash J} \mathfrak{m}\left(E_{i}\right)^{k-1 / k} \stackrel{(1.9)}{\leq} C_{I}^{\prime} \mathrm{P}(E)<+\infty .
$$

Hence, there exists $i \in I$ such that $\mathfrak{m}\left(E_{i}\right)=+\infty$. Suppose by contradiction to have $\mathfrak{m}\left(E_{j}\right)=$ $+\infty$ for some $j \in I \backslash\{i\}$. Then $E_{j} \subset E_{i}^{c}$ and accordingly $\mathfrak{m}\left(E_{i}^{c}\right)=+\infty$, which is not possible as we have that $\min \left\{\mathfrak{m}\left(E_{i}\right), \mathfrak{m}\left(E_{i}^{c}\right)\right\} \leq C_{I}^{\prime} \mathrm{P}\left(E_{i}\right)^{k / k-1}<+\infty$ by Theorem 1.18. The statement follows.

Remark 3.15 The Ahlfors-regularity assumption in Proposition 3.14 cannot be dropped, as shown by the following example. Let us consider the strip $X:=\mathbb{R} \times[0,1] \subset \mathbb{R}^{2}$, endowed with the (restricted) Euclidean distance and the 2-dimensional Hausdorff measure, which is an isotropic PI space. Then the square $E:=[0,1]^{2} \subset \mathrm{X}$ is an indecomposable set having finite measure, but its complement consists of two essential connected components having infinite measure.

Remark 3.16 If $(\mathrm{X}, d, \mathfrak{m})$ is a $k$-Ahlfors regular, isotropic PI space with $k>1$ and $\mathfrak{m}(\mathrm{X})=$ $+\infty$, then for any indecomposable set $E \subset \mathrm{X}$ with $\mathfrak{m}(E)<+\infty$ it holds that $\mathfrak{m}(\operatorname{sat}(E))<$ $+\infty$.

Indeed, we know that $\mathfrak{m}\left(\operatorname{sat}(E)^{c}\right)=+\infty$ by Proposition 3.14, whence the set $\operatorname{sat}(E)$ must have finite $\mathfrak{m}$-measure (otherwise we would contradict Theorem 1.18).

Theorem 3.17 (Simple sets on Ahlfors-regular spaces) Let $(\mathrm{X}, d, \mathfrak{m})$ be a $k$-Ahlfors regular PI space with $k>1$ and $\mathfrak{m}(\mathrm{X})=+\infty$. Suppose $(\mathrm{X}, d, \mathfrak{m})$ has the two-sidedness property. Let $E \subset \mathrm{X}$ be a set of finite perimeter with $\mathfrak{m}(E)<+\infty$. Then $E$ is simple if and only if it is both indecomposable and saturated.

Proof Necessity stems from Remark 3.11. To prove sufficiency, suppose that $E$ is indecomposable and saturated. Proposition 3.14 grants that $E^{c}$ is the unique element of $\mathcal{C C}^{e}(E)$ having infinite $\mathfrak{m}$-measure, thus in particular $E^{c}$ is indecomposable. By applying item (ii) of Proposition 3.2, we finally conclude that the set $E$ is simple, as desired.

\section{Alternative proof of the decomposition theorem}

We provide here an alternative proof of the Decomposition Theorem 2.14, in the particular case in which the set under consideration is bounded (the boundedness assumption is added 
for simplicity, cf. Remark 4.6 for a few comments about the unbounded case). The inspiration for this approach is taken from [33]. We refer to "Appendix B" for the language and the results we are going to use in this section.

Let $(\mathrm{X}, d, \mathfrak{m})$ be an isotropic PI space. Given any open set $\Omega \subset \mathrm{X}$ and any set $E \subset \Omega$ having finite perimeter in $\mathrm{X}$, we define the family $\Xi_{\Omega}(E)$ as

$$
\Xi_{\Omega}(E):=\{F \subset E \text { of finite perimeter in } \mathrm{X} \mid \mathrm{P}(E, \Omega)=\mathrm{P}(F, \Omega)+\mathrm{P}(E \backslash F, \Omega)\} .
$$

Observe that $\Xi_{\Omega}(E)=\Xi_{\Omega^{\prime}}(E)$ holds whenever $\Omega, \Omega^{\prime} \subset \mathrm{X}$ are open sets with $E \Subset \Omega$ and $E \Subset \Omega^{\prime}$.

Remark 4.1 It holds that $E$ is indecomposable in $\Omega$ if and only if $\Xi_{\Omega}(E)$ is trivial, i.e.,

$$
\Xi_{\Omega}(E)=\{F \subset E \text { Borel } \mid \mathfrak{m}(F)=0 \text { or } \mathfrak{m}(E \backslash F)=0\} .
$$

The proof of this fact is a direct consequence of the very definition of indecomposable set.

Lemma 4.2 Let $(\mathrm{X}, d, \mathfrak{m})$ be an isotropic PI space. Let $\Omega \subset \mathrm{X}$ be an open set with $\mathcal{H}(\partial \Omega)<$ $+\infty$. Let $E \subset \Omega$ be a set of finite perimeter in X. Then $\Xi_{\Omega}(E)$ is a $\sigma$-algebra of Borel subsets of $E$. Moreover, if $E \Subset \Omega$, then the assumption $\mathcal{H}(\partial \Omega)<+\infty$ can be dropped.

Proof Trivially, we have that $E \in \Xi_{\Omega}(E)$ and $\Xi_{\Omega}(E)$ is closed under complement. Moreover, fix any two sets $F, G \in \Xi_{\Omega}(E)$. Since $P(E, \Omega)=\mathrm{P}(F, \Omega)+\mathrm{P}(E \backslash F, \Omega)=\mathrm{P}(G, \Omega)+$ $\mathrm{P}(E \backslash G, \Omega)$, we deduce that $\mathrm{P}(G, \Omega)=\mathrm{P}(F \cap G, \Omega)+\mathrm{P}(G \backslash F, \Omega)$ and $\mathrm{P}(E \backslash G, \Omega)=$ $\mathrm{P}(F \backslash G, \Omega)+\mathrm{P}(E \backslash(F \cup G), \Omega)$ by Lemma 2.8. Consequently, the subadditivity of the perimeter yields

$$
\begin{aligned}
\mathrm{P}(E, \Omega) & \leq \mathrm{P}(F \cup G, \Omega)+\mathrm{P}(E \backslash(F \cup G), \Omega) \\
& \leq \mathrm{P}(F \cap G, \Omega)+\mathrm{P}(G \backslash F, \Omega)+\mathrm{P}(F \backslash G, \Omega)+\mathrm{P}(E \backslash(F \cup G), \Omega) \\
& =\mathrm{P}(G, \Omega)+\mathrm{P}(E \backslash G, \Omega)=\mathrm{P}(E, \Omega) .
\end{aligned}
$$

This forces the equality $\mathrm{P}(E, \Omega)=\mathrm{P}(F \cup G, \Omega)+\mathrm{P}(E \backslash(F \cup G), \Omega)$. Given that $F \cup G$ has finite perimeter, we have proved that $F \cup G \in \Xi_{\Omega}(E)$. This shows that $\Xi_{\Omega}(E)$ is closed under finite unions. Finally, to prove that $\Xi_{\Omega}(E)$ is closed under countable unions, fix any $\left(F_{i}\right)_{i} \subset \Xi_{\Omega}(E)$. Calling $F:=\bigcup_{i \in \mathbb{N}} F_{i}$, we aim to prove that $F \in \Xi_{\Omega}(E)$. We denote $F_{i}^{\prime}:=F_{1} \cup \cdots \cup F_{i} \in \Xi_{\Omega}(E)$ for all $i \in \mathbb{N}$. Given that $F=\bigcup_{i \in \mathbb{N}} F_{i}^{\prime}$, we have $\mathbb{1}_{F_{i}^{\prime}} \rightarrow \mathbb{1}_{F}$ and $\mathbb{1}_{E \backslash F_{i}^{\prime}} \rightarrow \mathbb{1}_{E \backslash F}$ in $L_{\text {loc }}^{1}\left(\mathfrak{m}_{\llcorner\Omega}\right)$. Hence, by lower semicontinuity and subadditivity of the perimeter we can conclude that

$$
\begin{aligned}
\mathrm{P}(E, \Omega) & \leq \mathrm{P}(F, \Omega)+\mathrm{P}(E \backslash F, \Omega) \leq \varliminf_{i \rightarrow \infty} \mathrm{P}\left(F_{i}^{\prime}, \Omega\right)+\varliminf_{i \rightarrow \infty} \mathrm{P}\left(E \backslash F_{i}^{\prime}, \Omega\right) \\
& \leq \varliminf_{i \rightarrow \infty}\left(\mathrm{P}\left(F_{i}^{\prime}, \Omega\right)+\mathrm{P}\left(E \backslash F_{i}^{\prime}, \Omega\right)\right)=\mathrm{P}(E, \Omega),
\end{aligned}
$$

which forces $\mathrm{P}(E, \Omega)=\mathrm{P}(F, \Omega)+\mathrm{P}(E \backslash F, \Omega)$. Notice also that $\mathbb{1}_{F_{i}^{\prime}} \rightarrow \mathbb{1}_{F}$ in $L_{\text {loc }}^{1}(\mathfrak{m})$, whence

$$
\begin{aligned}
\mathrm{P}(F) & \leq \varliminf_{i \rightarrow \infty} \mathrm{P}\left(F_{i}^{\prime}\right)=\varliminf_{i \rightarrow \infty}\left(\mathrm{P}\left(F_{i}^{\prime}, \Omega\right)+\mathrm{P}\left(F_{i}^{\prime}, \partial \Omega\right)\right) \leq \mathrm{P}(E, \Omega)+\varliminf_{i \rightarrow \infty}\left(\theta_{F_{i}^{\prime}} \mathcal{H}\right)(\partial \Omega) \\
& \leq \mathrm{P}(E, \Omega)+C_{D} \mathcal{H}(\partial \Omega)<+\infty .
\end{aligned}
$$

This says that the set $F$ has finite perimeter in X, thus $F$ belongs to $\Xi_{\Omega}(E)$, as desired. 
To prove the last statement, let us assume that $E \Subset \Omega$. By exploiting Remark 1.20 and the boundedness of $E$, we can find an open ball $B \subset \mathrm{X}$ such that $E \Subset B$ and $\mathcal{H}(\partial B)<+\infty$, thus accordingly the family $\Xi_{\Omega}(E)=\Xi_{B}(E)$ is a $\sigma$-algebra by the previous part of the proof.

Remark 4.3 Let $(\mathrm{X}, d, \mathfrak{m})$ be an isotropic PI space and $\Omega \subset \mathrm{X}$ an open set. Then we claim that

$$
\begin{aligned}
\Xi_{\Omega}(G) & =\left\{F \in \Xi_{\Omega}(E) \mid F \subset G\right\} \quad \text { for every } E \subset \Omega \text { Borel with } \mathrm{P}(E) \\
& <+\infty \text { and } G \in \Xi_{\Omega}(E) .
\end{aligned}
$$

We separately prove the two inclusions. Fix $F \in \Xi_{\Omega}(G)$. Since $\mathrm{P}(E, \Omega)=\mathrm{P}(G, \Omega)+$ $\mathrm{P}(E \backslash G, \Omega)$, we know from Lemma 2.8 that $\mathrm{P}(E \backslash F, \Omega)=\mathrm{P}(G \backslash F, \Omega)+\mathrm{P}(E \backslash G, \Omega)$. Therefore, we have that

$$
\begin{aligned}
\mathrm{P}(E, \Omega) & =\mathrm{P}(G, \Omega)+\mathrm{P}(E \backslash G, \Omega)=\mathrm{P}(F, \Omega)+\mathrm{P}(G \backslash F, \Omega)+\mathrm{P}(E \backslash G, \Omega) \\
& =\mathrm{P}(F, \Omega)+\mathrm{P}(E \backslash F, \Omega),
\end{aligned}
$$

thus proving that $F \in \Xi_{\Omega}(E)$. Conversely, let us fix any set $F^{\prime} \in \Xi_{\Omega}(E)$ such that $F^{\prime} \subset G$. Given that $\mathrm{P}(E, \Omega)=\mathrm{P}\left(F^{\prime}, \Omega\right)+\mathrm{P}\left(E \backslash F^{\prime}, \Omega\right)$, we conclude that $\mathrm{P}(G, \Omega)=\mathrm{P}\left(F^{\prime}, \Omega\right)+$ $\mathrm{P}\left(G \backslash F^{\prime}, \Omega\right)$ again by Lemma 2.8 . This shows that $F^{\prime} \in \Xi_{\Omega}(G)$, which yields the sought conclusion.

Lemma 4.4 Let $(\mathrm{X}, d, \mathfrak{m})$ be an isotropic PI space. Let $\Omega \subset \mathrm{X}$ be an open set. Let $E \subset \Omega$ be a set of finite perimeter in $\mathrm{X}$. Then for any finite partition $\left\{E_{1}, \ldots, E_{n}\right\} \subset \Xi_{\Omega}(E)$ of the set $E$ it holds that $P(E, \Omega)=P\left(E_{1}, \Omega\right)+\cdots+P\left(E_{n}, \Omega\right)$.

Proof Recall that $\mathrm{P}(E, \Omega)=\mathrm{P}\left(E_{i}, \Omega\right)+\mathrm{P}\left(E \backslash E_{i}, \Omega\right)$ for all $i=1, \ldots, n$, thus by repeatedly applying Lemma 2.8 we obtain that

$$
\mathrm{P}(E, \Omega)=\mathrm{P}\left(E_{1}, \Omega\right)+\mathrm{P}\left(E_{2} \cup \cdots \cup E_{n}, \Omega\right)=\cdots=\mathrm{P}\left(E_{1}, \Omega\right)+\cdots+\mathrm{P}\left(E_{n}, \Omega\right) .
$$

Therefore, the statement is achieved.

Theorem 4.5 Let $(\mathrm{X}, d, \mathfrak{m})$ be an isotropic PI space. Let $\Omega \subset \mathrm{X}$ be an open set. Then the measure space $\left(E, \Xi_{\Omega}(E), \mathfrak{m}_{\llcorner E}\right)$ is purely atomic for every bounded set $E \Subset \Omega$ of finite perimeter.

Proof We can assume without loss of generality that $\Omega=B_{r}(\bar{x})$ for some $\bar{x} \in \mathrm{X}$ and $r>0$. For the sake of brevity, let us denote $\mathbb{M}_{F}:=\left(F, \Xi_{\Omega}(F), \mathfrak{m}_{\llcorner F}\right)$ for every $F \in \Xi_{\Omega}(E)$. It follows from Remark 4.3 that $\Xi_{\Omega}(E)\left\llcorner F=\Xi_{\Omega}(F)\right.$ and that the atoms of $\mathbb{M}_{F}$ coincide with the atoms of $\mathbb{M}_{E}$ that are contained in $F$. Accordingly, in order to prove that $\mathbb{M}_{E}$ is purely atomic, it suffices to show that $\mathbb{M}_{F}$ is atomic for any set $F \in \Xi_{\Omega}(E)$ with $\mathfrak{m}(F)>0$. We argue by contradiction: suppose $\mathbb{M}_{F}$ is non-atomic. Let us fix any $\varepsilon>0$. Corollary B.5 grants that there exists a finite partition $\left\{F_{1}, \ldots, F_{n}\right\} \subset \Xi_{\Omega}(F)$ of $F$ such that $\mathfrak{m}\left(F_{i}\right) \leq \min \left\{\varepsilon, \mathfrak{m}\left(F \backslash F_{i}\right)\right\}$ for all $i=1, \ldots, n$. Let us apply Theorem 1.17: calling $C$ the quantity $C_{I}\left(r^{s} / \mathfrak{m}\left(B_{r}(\bar{x})\right)\right)^{1 / s-1}$, one has that

$$
\left(\frac{\mathfrak{m}\left(F_{i}\right)}{C}\right)^{s-1 / s} \leq \mathrm{P}\left(F_{i}, B_{2 \lambda r}(\bar{x})\right)=\mathrm{P}\left(F_{i}, \Omega\right) \quad \text { for every } i=1, \ldots, n .
$$

Since $\sum_{i=1}^{n} \mathrm{P}\left(F_{i}, \Omega\right)=\mathrm{P}(F, \Omega)$ holds by Lemma 4.4, we deduce from the previous inequality that 


$$
\frac{\mathfrak{m}(F)}{C^{s-1 / s} \varepsilon^{1 / s}} \leq \frac{1}{C} \sum_{i=1}^{n} \mathfrak{m}\left(F_{i}\right)\left(\frac{\mathfrak{m}\left(F_{i}\right)}{C}\right)^{-1 / s}=\sum_{i=1}^{n}\left(\frac{\mathfrak{m}\left(F_{i}\right)}{C}\right)^{s-1 / s} \leq \mathrm{P}(F, \Omega) .
$$

By letting $\varepsilon \searrow 0$ in (4.1) we get that $\mathrm{P}(F, \Omega)=+\infty$, which yields a contradiction. Therefore, we conclude that the measure space $\mathbb{M}_{F}$ is non-atomic, as required.

Alternative proof of Theorem 2.14 for E bounded. Maximality and uniqueness can be proven as in Proposition 2.13, thus we can just focus on the existence part of the statement. The measure space $\left(E, \Xi_{X}(E), \mathfrak{m}_{\llcorner E}\right)$ is purely atomic by Theorem 4.5 , thus there exists an at most countable family of pairwise disjoint atoms $\left\{E_{i}\right\}_{i \in I} \subset \Xi_{X}(E)$ such that $\mathfrak{m}\left(E \backslash \bigcup_{i \in i} E_{i}\right)=0$ by Remark B.2. Moreover, we deduce from Remark 4.3 that each set $E_{i}$ is an atom of $\left(E_{i}, \Xi_{\mathbf{X}}\left(E_{i}\right), \mathfrak{m}_{\left\llcorner E_{i}\right.}\right)$, which is clearly equivalent to saying that $\Xi_{X}\left(E_{i}\right)$ is trivial (in the sense of Remark 4.1). Accordingly, the set $E_{i}$ is indecomposable for every $i \in I$. Finally, Lemma 4.4 grants that $\mathrm{P}(E)=\sum_{i \in I} \mathrm{P}\left(E_{i}\right)$.

Remark 4.6 Let us briefly outline how to prove the decomposition theorem via Theorem B.3 in the general case (i.e., when $E$ is possibly unbounded). More specifically, we show that the existence part of Proposition 2.13 (under the additional assumption that $\partial B_{r}(\bar{x})$ has finite $\mathcal{H}$-measure) can be deduced from Theorem B.3, whence Theorem 2.14 follows (thanks to Remark 1.20).

Our aim is to show that $\left(E \cap \Omega, \Xi_{\Omega}(E \cap \Omega), \mathfrak{m}_{\llcorner E \cap \Omega}\right)$ is purely atomic, where we set $\Omega:=B_{r}(\bar{x})$. We argue by contradiction: suppose $\left(F, \Xi_{\Omega}(F), \mathfrak{m}_{\llcorner F}\right)$ is non-atomic for some $F \in \Xi_{\Omega}(E \cap \Omega)$. Then Corollary B.5, Theorems 1.17 and 1.23 ensure that for any $\varepsilon>0$ there exist a finite partition $\left\{F_{1}, \ldots, F_{n_{\varepsilon}}\right\} \subset \Xi_{\Omega}(F)$ of $F$ and a constant $c>0$ such that $\mathfrak{m}\left(F_{1}\right), \ldots, \mathfrak{m}\left(F_{n_{\varepsilon}}\right) \leq \varepsilon$ and

$$
c \mathfrak{m}\left(F_{i}\right)^{s-1 / s} \leq \mathrm{P}\left(F_{i}, \Omega\right)+C_{D} \mathcal{H}\left(\Sigma_{\tau}\left(F_{i}\right) \cap \partial \Omega\right) \quad \text { for every } i=1, \ldots, n_{\varepsilon},
$$

where the set $\Sigma_{\tau}\left(F_{i}\right)$ is defined as in (1.13). Given any $\ell \in \mathbb{N}$ such that $\ell \tau>1$, it is clear that the set $\bigcap_{i \in S} \Sigma_{\tau}\left(F_{i}\right)$ is empty whenever we choose $S \subset\left\{1, \ldots, n_{\varepsilon}\right\}$ of cardinality greater than $\ell$. Therefore, we deduce from (4.2) and the identity $\sum_{i=1}^{n_{\varepsilon}} \mathrm{P}\left(F_{i}, \Omega\right)=\mathrm{P}(F, \Omega)$ that

$$
c \frac{\mathfrak{m}(F)}{\varepsilon^{1 / s}}=c \sum_{i=1}^{n_{\varepsilon}} \frac{\mathfrak{m}\left(F_{i}\right)}{\varepsilon^{1 / s}} \leq c \sum_{i=1}^{n_{\varepsilon}} \mathfrak{m}\left(F_{i}\right)^{s-1 / s} \leq \mathrm{P}(F, \Omega)+C_{D} \mathcal{H}(\partial \Omega) \ell
$$

Finally, by letting $\varepsilon \searrow 0$ we conclude that $\mathrm{P}(F, \Omega)=+\infty$, which leads to a contradiction.

Acknowledgements Open access funding provided by University of Jyväskylä (JYU). The first named author acknowledges ERC Starting Grant 676675 FLIRT. The second and third named authors are partially supported by the Academy of Finland, Projects 274372, 307333, 312488, and 314789. The authors would like to thank Panu Lahti for many useful comments on the paper.

Open Access This article is licensed under a Creative Commons Attribution 4.0 International License, which permits use, sharing, adaptation, distribution and reproduction in any medium or format, as long as you give appropriate credit to the original author(s) and the source, provide a link to the Creative Commons licence, and indicate if changes were made. The images or other third party material in this article are included in the article's Creative Commons licence, unless indicated otherwise in a credit line to the material. If material is not included in the article's Creative Commons licence and your intended use is not permitted by statutory regulation or exceeds the permitted use, you will need to obtain permission directly from the copyright holder. To view a copy of this licence, visit http://creativecommons.org/licenses/by/4.0/. 


\section{Appendix A: Extreme points}

Let $V$ be a normed space. Let $K \neq \varnothing$ be a convex, compact subset of $V$. Then we shall denote by ext $K$ the set of all extreme points of $K$, namely of those points $x \in K$ that cannot be written as $x=t y+(1-t) z$ for some $t \in(0,1)$ and some distinct $y, z \in K$. The Krein-Milman theorem states that $K$ coincides with the closed convex hull of ext $K$; cf. [34]. Furthermore, it actually holds that ext $K$ is the 'smallest' set having this property:

Theorem A.1 (Milman [37]) Let $V$ be a normed space. Let $\emptyset \neq K \subset V$ be convex and compact. Suppose that the closed convex hull of a set $S \subset K$ coincides with $K$. Then ext $K$ is contained in the closure of $S$.

Another fundamental result in functional analysis and convex analysis is the following celebrated strengthening of the Krein-Milman theorem:

Theorem A.2 (Choquet [39]) Let $V$ be a normed space. Let $\emptyset \neq K \subset V$ be convex and compact. Then for any point $x \in K$ there exists a Borel probability measure $\mu$ on $V$ (depending on $x$ ), which is concentrated on ext $K$ and satisfies

$$
L(x)=\int L(y) \mathrm{d} \mu(y) \text { for every } L: V \rightarrow \mathbb{R} \text { linear and continuous. }
$$

Remark A.3 In the above result, the measure $\mu$ is concentrated on ext $K$. For completeness, we briefly verify that ext $K$ is a Borel subset of $V$ : the set $K \backslash$ ext $K$ can be written as $\bigcup_{n} C_{n}$, where

$$
C_{n}:=\left\{\frac{y+z}{2} \mid y, z \in K,\|y-z\|_{V} \geq 1 / n\right\} \quad \text { for every } n \in \mathbb{N} .
$$

Given that each set $C_{n}$ is a closed subset of $V$, we conclude that ext $K$ is Borel.

\section{Appendix B: Lyapunov vector-measure theorem}

In the theory of vector measures, an important role is played by the following theorem (due to Lyapunov): the range of a non-atomic vector measure is closed and convex; cf., for instance, [20]. For our purposes, we need a simpler version of this theorem (just for scalar measures). For the reader's convenience, we report below (see Theorem B.3) an elementary proof of this result.

Let us begin by recalling the definition of atom in a measure space (see also [12]):

Definition B.1 (Atom) Let $(\mathrm{X}, \mathcal{A}, \mu)$ be a measure space. Then a set $A \in \mathcal{A}$ with $\mu(A)>0$ is said to be an atom of $\mu$ provided for any set $A^{\prime} \in \mathcal{A}$ with $A^{\prime} \subset A$ it holds that either $\mu\left(A^{\prime}\right)=0$ or $\mu\left(A \backslash A^{\prime}\right)=0$. The measure space $(\mathrm{X}, \mathcal{A}, \mu)$ is called non-atomic if there are no atoms, atomic if there exists at least one atom, and purely atomic if every measurable set of positive $\mu$-measure contains an atom.

Remark B.2 Given a purely atomic measure space $(\mathrm{X}, \mathcal{A}, \mu)$ and a set $E \in \mathcal{A}$ such that $\mu(E)>0$, there exists an at most countable family $\left\{A_{i}\right\}_{i \in I} \subset \mathcal{A}$ of pairwise disjoint atoms of $\mu$, which are contained in $E$ and satisfy $\mu\left(E \backslash \bigcup_{i \in I} A_{i}\right)=0$; cf. [32, Theorem 2.2].

Recall that a measure space $(\mathrm{X}, \mathcal{A}, \mu)$ is semifinite provided for every set $E \in \mathcal{A}$ with $\mu(E)>0$ there exists $F \in \mathcal{A}$ such that $F \subset E$ and $0<\mu(F)<+\infty$. 
Theorem B.3 (Non-atomic measures have full range) Let $(\mathrm{X}, \mathcal{A}, \mu)$ be a semifinite, nonatomic measure space. Then for every constant $\lambda \in(0, \mu(\mathrm{X}))$ there exists $A \in \mathcal{A}$ such that $\mu(A)=\lambda$.

Proof First of all, let us prove the following claim:

Given any set $A \in \mathcal{A}$ with $\mu(A)>0$ and any $\varepsilon>0$, there exists $B \in \mathcal{A}$ such that $B \subset A$ and $0<\mu(B)<\varepsilon$.

In order to prove it, fix a subset $A^{\prime} \in \mathcal{A}$ of $A$ with $0<\mu\left(A^{\prime}\right)<+\infty$ (whose existence follows from the semifiniteness assumption) and any $k \in \mathbb{N}$ such that $k>\mu\left(A^{\prime}\right) / \varepsilon$. Since $\mu$ admits no atoms, we can find a partition $B_{1}, \ldots, B_{k} \in \mathcal{A}$ of $A^{\prime}$ such that $\mu\left(B_{i}\right)>0$ for every $i=1, \ldots, k$. Hence, there must exist $i=1, \ldots, k$ such that $\mu\left(B_{i}\right)<\varepsilon$, otherwise we would have that

$$
\mu\left(A^{\prime}\right)=\mu\left(B_{1}\right)+\cdots+\mu\left(B_{k}\right) \geq k \varepsilon>\mu\left(A^{\prime}\right) .
$$

Therefore, the set $B:=B_{i}$ satisfies $B \subset A^{\prime} \subset A$ and $0<\mu(B)<\varepsilon$. This proves the claim (B.1).

We recursively build a sequence $\left(A_{n}\right)_{n} \subset \mathcal{A}$. The set $A_{1}$ is any element of $\mathcal{A}$ with $0<$ $\mu\left(A_{1}\right)<\lambda$, which can be found thanks to (B.1). Now let us suppose to have already defined $A_{1}, \ldots, A_{n-1}$ for some natural number $n \geq 2$ with the following properties: $A_{1}, \ldots, A_{n-1} \in$ $\mathcal{A}$ are pairwise disjoint sets that satisfy $\mu\left(A_{1}\right), \ldots, \mu\left(A_{n-1}\right)>0$ and $\sum_{i=1}^{n-1} \mu\left(A_{i}\right)<\lambda$. We set

$$
\mathcal{F}_{n}:=\left\{B \in \mathcal{A} \mid B \subset \mathrm{X} \backslash \bigcup_{i=1}^{n-1} A_{i}, 0<\mu(B)<\lambda-\sum_{i=1}^{n-1} \mu\left(A_{i}\right)\right\} .
$$

Property (B.1) grants that $\mathcal{F}_{n}$ is non-empty, thus in particular $s_{n}:=\sup \left\{\mu(B) \mid B \in \mathcal{F}_{n}\right\}>0$. Let $A_{n}$ be any element of $\mathcal{F}_{n}$ such that $\mu\left(A_{n}\right) \geq s_{n} / 2$. Notice that $A_{1}, \ldots, A_{n} \in \mathcal{A}$ are pairwise disjoint sets of positive $\mu$-measure for which $\mu\left(A_{1}\right)+\cdots+\mu\left(A_{n}\right)<\lambda$.

Now let us call $A:=\bigcup_{n=1}^{\infty} A_{n} \in \mathcal{A}$. We argue by contradiction: suppose that $\mu(A) \neq \lambda$. Given that $\mu(A)=\lim _{n} \sum_{i=1}^{n} \mu\left(A_{i}\right) \leq \lambda$, this means that $\mu(A)<\lambda$. We know from (B.1) that there exists a set $B \in \mathcal{A}$ with $B \subset \mathrm{X} \backslash A$ and $0<\mu(B)<\lambda-\mu(A)$. Since $\sum_{n=1}^{\infty} \mu\left(A_{n}\right)<\lambda<+\infty$, we can pick some $n \geq 1$ for which $\mu\left(A_{n}\right)<\mu(B) / 2$. On the other hand, one has that $B \subset \mathrm{X} \backslash A \subset \mathrm{X} \backslash \bigcup_{i=1}^{n-1} A_{i}$ and $0<\mu(B)<\lambda-\mu(A) \leq \lambda-\sum_{i=1}^{n-1} \mu\left(A_{i}\right)$, whence accordingly $B \in \mathcal{F}_{n}$. Consequently, it must hold that $\mu\left(A_{n}\right) \geq s_{n} / 2 \geq \mu(B) / 2$, which leads to a contradiction. We conclude that $\mu(A)=\lambda$, which finally yields the statement.

Remark B.4 Given a semifinite, non-atomic measure space $(\mathrm{X}, \mathcal{A}, \mu)$ and a set $E \in \mathcal{A}$, it holds that $\left(E, \mathcal{A}_{\llcorner E}, \mu_{\llcorner E}\right)$ is semifinite and non-atomic as well, where the restricted $\sigma$ algebra $\mathcal{A}_{\llcorner E}$ is defined as $\mathcal{A}_{\llcorner E}:=\{A \cap E: A \in \mathcal{A}\}$. In particular, one can readily deduce from Theorem B.3 that for any $\lambda \in(0, \mu(E))$ there exists $A \in \mathcal{A}_{\llcorner}$such that $\mu(A)=\lambda$.

Corollary B.5 Let $(\mathrm{X}, \mathcal{A}, \mu)$ be a finite, non-atomic measure space. Then for every $\varepsilon>0$ there exists a partition $\left\{A_{1}, \ldots, A_{n}\right\} \subset \mathcal{A}$ of $\mathrm{X}$ such that $\mu\left(A_{i}\right) \leq \min \left\{\varepsilon, \mu\left(A_{i}^{c}\right)\right\}$ for all $i=1, \ldots, n$.

Proof Fix any $\varepsilon^{\prime}>0$ such that $\varepsilon^{\prime}<\varepsilon$ and $\varepsilon^{\prime}<\mu(\mathrm{X}) / 2$. We proceed in a recursive way: first of all, choose a set $A_{1} \in \mathcal{A}$ with $\mu\left(A_{1}\right)=\varepsilon^{\prime}$, whose existence is granted by 
Theorem B.3. Now we can pick a set $A_{2} \in \mathcal{A}_{\left\llcorner A_{1}^{c}\right.}$ such that $\mu\left(A_{2}\right)=\varepsilon^{\prime}$ (recall Remark B.4). After finitely many steps, we end up with pairwise disjoint measurable sets $A_{1}, \ldots, A_{n-1}$ such that $\mu\left(\mathrm{X} \backslash\left(A_{1} \cup \cdots \cup A_{n-1}\right)\right)<\varepsilon^{\prime}$. Let us define $A_{n}:=\mathrm{X} \backslash\left(A_{1} \cup \ldots \cup A_{n-1}\right) \in \mathcal{A}$. Therefore, the sets $A_{1}, \ldots, A_{n}$ do the job.

\section{References}

1. Ambrosio, L.: Some fine properties of sets of finite perimeter in Ahlfors regular metric measure spaces. Adv. Math. 159, 51-67 (2001)

2. Ambrosio, L.: Fine properties of sets of finite perimeter in doubling metric measure spaces. Set-Valued Anal. 10, 111-128 (2002)

3. Ambrosio, L., Bruè, E., Semola, D.: Rigidity of the 1-Bakry-Émery inequality and sets of finite perimeter in RCD spaces. Geom. Funct. Anal. 29, 949-1001 (2019). https://doi.org/10.1007/s00039-019-00504-5

4. Ambrosio, L., Caselles, V., Masnou, S., Morel, J.-M.: Connected components of sets of finite perimeter and applications to image processing. J. Eur. Math. Soc. 3, 39-92 (2001)

5. Ambrosio, L., Di Marino, S.: Equivalent definitions of BV space and of total variation on metric measure spaces. J. Funct. Anal. 266, 4150-4188 (2014)

6. Ambrosio, L., Fusco, N., Pallara, D.: Functions of Bounded Variation and Free Discontinuity Problems. Oxford Science Publications, Clarendon Press, Oxford (2000)

7. Ambrosio, L., Miranda, M., Pallara, D.: Special functions of bounded variation in doubling metric measure spaces. Quad. Mat. 14, 1-45 (2004)

8. Ambrosio, L., Scienza, M.: Locality of the perimeter in Carnot groups and chain rule. Ann. Mat. Pura Appl. 189, 661-678 (2010)

9. Baldi, A.: Weighted BV functions. Houston Math. J. 27, 683-705 (2001)

10. Bellettini, G., Bouchitté, G., Fragalà, I.: BV functions with respect to a measure and relaxation of metric integral functionals. J. Convex Anal. 6, 349-366 (1999)

11. Biroli, M., Mosco, U.: Sobolev and isoperimetric inequalities for Dirichlet forms on homogeneous spaces. Atti Accad. Naz. Lincei. Cl. Sci. Fis. Mat. Nat. 6, 37-44 (1995)

12. Bogachev, V.I.: Measure Theory. Springer, Berlin (2007)

13. Bonicatto, P., Gusev, N.A.: On the structure of divergence-free measures in $\mathbb{R}^{2}$, Submitted (2019). arXiv: 1912.10936

14. Bruè, E., Pasqualetto, E., Semola, D.: Rectifiability of the reduced boundary for sets of finite perimeter over $\operatorname{RCD}(K, N)$ spaces. Submitted (2019). arXiv:1909.00381

15. Caccioppoli, R.: Misura e integrazione sugli insiemi dimensionalmente orientati, Accad. Naz. Lincei 12, pp. 3-11, 137-146 (1952)

16. Danielli, D., Garofalo, N., Nhieu, D.-M.: Trace inequalities for Carnot-Carathéodory spaces and applications. Ann. Scuola Norm. Sup. 27, 195-252 (1998)

17. De Giorgi, E.: Su una teoria generale della misura $(r-1)$-dimensionale in uno spazio ad $r$ dimensioni. Ann. Mat. Pura Appl. 36, 191-213 (1954)

18. Di Marino, S.: Recent advances on BV and Sobolev spaces in metric measure spaces (2014). Ph.D. Thesis

19. Di Marino, S.: Sobolev and BV spaces on metric measure spaces via derivations and integration by parts (2014). Submitted paper, arXiv: 1409.5620

20. Diestel, J., Uhl, J.J.: Vector Measures. American Mathematical Society, Mathematical Surveys and Monographs (1977)

21. Dolzmann, G., Müller, S.: Microstructures with finite surface energy: the two-well problem. Arch. Ration. Mech. Anal. 132, 101-141 (1995)

22. Federer, H.: Geometric Measure Theory. Grundlehren der mathematischen Wissenschaften. Springer, New York (1969)

23. Fleming, W.H.: Functions with generalized gradient and generalized surfaces. Ann. Mat. Pura Appl. 44, 93-103 (1957)

24. Fleming, W.H.: Functions whose partial derivatives are measures. Ill. J. Math. 4, 452-478 (1960)

25. Franchi, B., Serapioni, R., Serra Cassano, F.: Meyers-Serrin type theorems and relaxation of variational integrals depending on vector fields. Houst. Math. J. 22, 859-889 (1996)

26. Franchi, B., Serapioni, R., Serra Cassano, F.: Rectifiability and perimeter in the Heisenberg group. Math. Ann. 321, 479-531 (2001)

27. Franchi, B., Serapioni, R., Serra Cassano, F.: On the structure of finite perimeter sets in step 2 Carnot groups. J. Geom. Anal. 13, 421 (2003) 
28. Garofalo, N., Nhieu, D.-M.: Isoperimetric and Sobolev inequalities for Carnot-Carathéodory spaces and the existence of minimal surfaces. Commun. Pure Appl. Math. 49, 1081-1144 (1996)

29. Giusti, E.: Minimal Surfaces and Functions of Bounded Variation. Birkhäuser, Basel (1985)

30. Heinonen, J., Koskela, P.: Quasiconformal maps in metric spaces with controlled geometry. Acta Math. 181, 1-61 (1998)

31. Heinonen, J., Koskela, P., Shanmugalingam, N., Tyson, J.T.: Sobolev Spaces on Metric Measure Spaces: An Approach Based on Upper Gradients, New Mathematical Monographs, vol. 27. Cambridge University Press, Cambridge (2015)

32. Johnson, R.A.: Atomic and nonatomic measures. Proc. Am. Math. Soc. 25, 650-655 (1970)

33. Kirchheim, B.: Lipschitz minimizers of the 3-well problem having gradients of bounded variation. Preprint 12, Max Planck Institute for Mathematics in the Sciences, Leipzig (1998)

34. Krein, M., Milman, D.: On extreme points of regular convex sets. Studia Math. 9, 133-138 (1940)

35. Mackay, J.M., Tyson, J.T., Wildrick, K.: Modulus and Poincaré inequalities on non-self-similar Sierpiński carpets. Geom. Funct. Anal. 23, 985-1034 (2013)

36. Mattila, P.: Geometry of Sets and Measures in Euclidean Spaces: Fractals and Rectifiability. Cambridge Studies in Advanced Mathematics. Cambridge University Press, Cambridge (1995)

37. Milman, D.: Characteristics of extreme points of regularly convex sets. Dokl. Akad. Nauk. SSSR 57, 119-122 (1947)

38. Miranda, M.: Functions of bounded variation on "good" metric spaces. J. Math. Pures Appl. 82, 975-1004 (2003)

39. Phelps, R.R.: Lectures on Choquet's Theorem, Lecture Notes in Mathematics. Springer, Berlin (2003)

Publisher's Note Springer Nature remains neutral with regard to jurisdictional claims in published maps and institutional affiliations. 